\title{
Phytodiversität auf verschiedenen räumlichen Skalenebenen am Beispiel des Messtischblattes 2728 / Lüneburg
}

Diplomarbeit im Studiengang Umweltwissenschaften

\author{
Vorgelegt von \\ Marc-André Allers \\ Leuphana Universität Lüneburg \\ Fakultät III - Umwelt und Technik \\ Institut für Ökologie und Umweltchemie
}

Juni 2007 

Marc-André Allers

Geboren am 15.05.1980

Matrikelnummer 2139418

Burmeisterstr. 5

21335 Lüneburg

Erstgutachter:

Dr. Jürgen Dengler

Leuphana Universität Lüneburg

Fakultät III: Umwelt und Technik

Institut für Ökologie und Umweltchemie

Scharnhorststr. 1, 21335 Lüneburg

Zweitgutachter:

Prof. Dr. Werner Härdtle

Leuphana Universität Lüneburg

Fakultät III: Umwelt und Technik

Institut für Ökologie und Umweltchemie

Scharnhorststr. 1, 21335 Lüneburg 
Hiermit erkläre ich, dass ich diese Arbeit eigenständig verfasst und keine anderen als die angegebenen Quellen und Hilfsmittel benutzt habe. 


\section{Danksagung}

Mein Dank gilt vor allen meinem Betreuer Dr. Jürgen Dengler für dessen unvergleichliche Betreuung, die geduldige Anleitung zur wissenschaftlichen Arbeit und die stete Hilfsbereitschaft. Von der Spezifizierung der Fragestellung und der Entwicklung des Aufnahmedesigns über die Bestimmung schwieriger Sippen bis zur Auswertung der Daten und zum Verfassen einer wissenschaftlichen Arbeit, konnte ich mich immer auf seine geduldige und kompetente Hilfe verlassen. Ich fühlte mich mit meinen Fragen stets willkommen und war nach jedem Gespräch voll positiver Energie.

Prof. Dr. Werner Härdtle danke ich dafür, dass er die Zweitbetreuung übernommen hat und ergänzende Hinweise geben konnte.

Beide Betreuer haben im Laufe zahlreicher Seminare und Exkursionen mein Interesse an der Flora geweckt und damit den Grundstein zu dieser Arbeit gelegt.

Praktische Hilfe wurde mir von vielen Seiten zu Teil. Manfred Gauger von der Behörde für Geoinformation, Landentwicklung und Liegenschaften, Lüneburg danke ich für die digitalen Orthophotos des kompletten Meßtischblattes 2728, Lüneburg. Hartmut Meyer danke ich für die Bereitstellung des Horizonthoskopes, Dieter Stengel für den GPS-Empfänger und die Plexiglasrahmen für die Erfassung der Artenzahlen auf kleinen Flächengrößen und Susanne WediPumpe für die Einweisung in die Laborarbeit sowie die Bereitstellung des Bandmaßes. Für die Hilfe bei der Bestimmung unbekannter Sippen möchte ich außerdem Steffen Boch meinen Dank aussprechen. Er hat mich in den letzten Jahren bei zahlreichen Gelegenheiten an seiner großen Artenkenntnis teilhaben lassen und mir viele wichtige Hinweise $\mathrm{zu}$ Artmerkmalen und Schwierigkeiten bei der Bestimmung kritischer Sippen gegeben.

Des weiteren danke ich meinen Freunden und Kommilitonen, die mit hilfreichen Ratschlägen und Korrekturvorschlägen einen essentiellen Beitrag zu dieser Arbeit geleistet haben.

Mein Studium wäre kaum möglich gewesen ohne die Hilfe meiner Eltern, die mich während der gesamten Zeit finanziell und moralisch unterstützt haben und mit steigendem Interesse meinen Werdegang verfolgen. Vielen Dank auch an sie für die Korrekturen rechtschreiblicher und grammatikalischer sowie die Verbesserung stilistischer Eskapaden.

Besonderer Dank gilt außerdem meiner Verlobten Sarah Wernicke, die während des ganzen Jahres viel von ihrer eigenen Zeit und Kraft direkt und indirekt in das Gelingen dieser Arbeit gesteckt hat. Sie hat mich nicht nur immer wieder unterstützt und motiviert, sondern mir durch ihre Begleitung bei Vegetationsaufnahmen und ihre unwiderstehlich fröhliche Art manch schwere Phase versüßt. 



\section{Inhaltsverzeichnis}

1. Einleitung und Fragestellungen........................................................................................... 1

2. Das Untersuchungsgebiet........................................................................................................... 4

2.1 Lage und allgemeine Gebietsbeschreibung............................................................................ 4

2.2 Klima.............................................................................................................................

2.3 Physische Geographie und Böden.............................................................................. 7

2.4 Nutzungs- und Kulturgeschichte...............................................................................................

2.5 Vegetation und Flora...................................................................................................................9

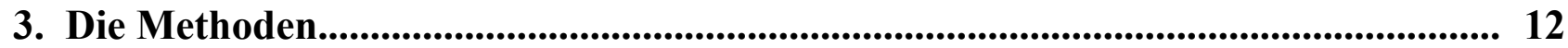

3.1 Vegetationskundliche Methoden........................................................................................... 12

3.1.1 Auswahl der Plots...................................................................................... 12

3.1.2 Aufnahmedesign...................................................................................... 14

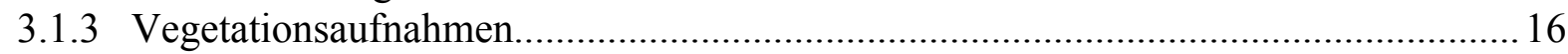

3.2 Begriffsdefinitionen............................................................................................................ 21

3.3 Datenauswertung................................................................................................................ 21

3.4 Statistische Verfahren.................................................................................................. 23

3.4.1 Artenzahlen auf unterschiedlichen Flächengrößen.................................................... 23

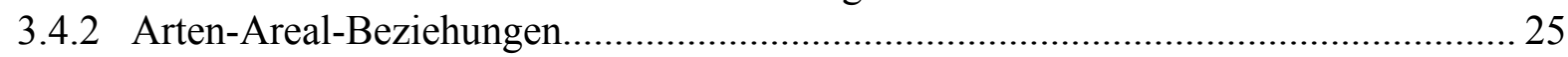

3.5 Nomenklatur und Bestimmungshilfen...............................................................................2 27

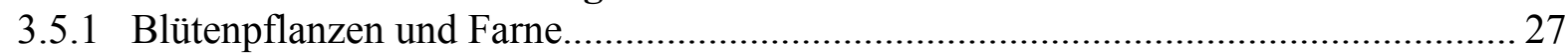

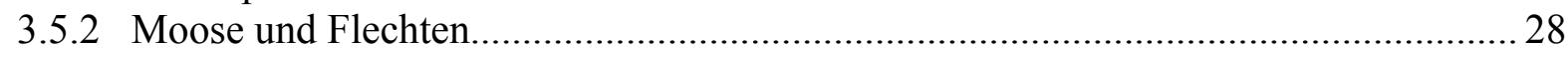

4. Artenzahlen auf unterschiedlichen Flächengrößen............................................................. 29

4.1 Absolute und mittlere Artenzahlen.....................................................................................29

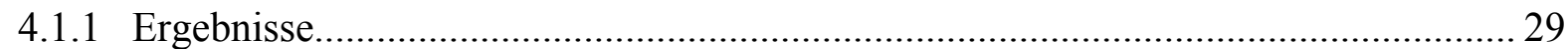

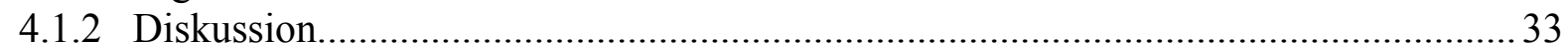

4.2 Verteilung der Artenzahlen................................................................................................. 38

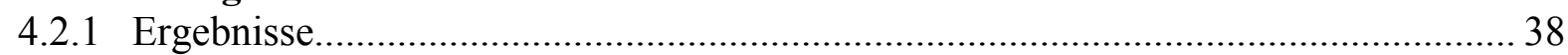

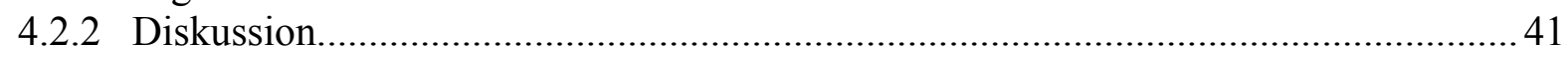

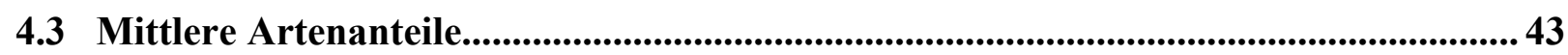

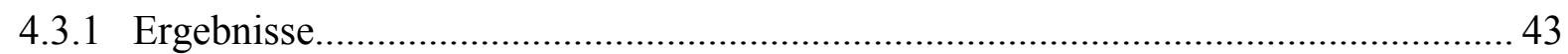

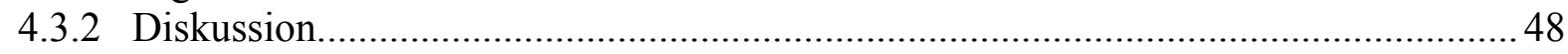

4.4 Zur Variabilität der Artenzahlen............................................................................49

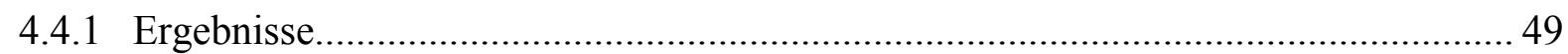

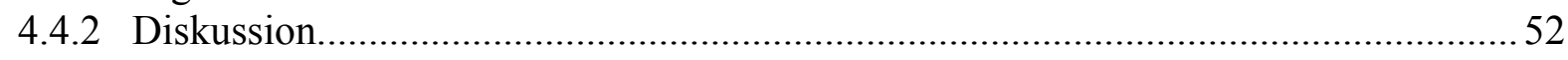

5. Arten-Areal-Beziehungen....................................................................................................... 54

5.1 Welche Funktion beschreibt den Zusammenhang von Artenzahl und Arealgröße am besten?..............................................................................................................................54

5.1.1 Ergebnisse

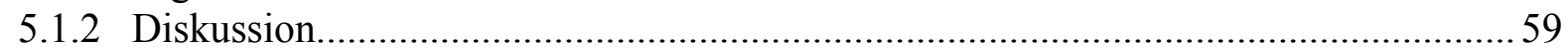

5.2 Zur Steigung der Artenzahl-Areal-Kurven..................................................................61

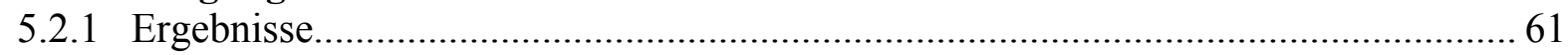

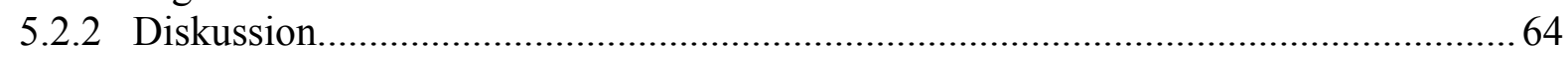


6. Häufigste Arten und Häufigkeitsverteilungen............................................................... 68

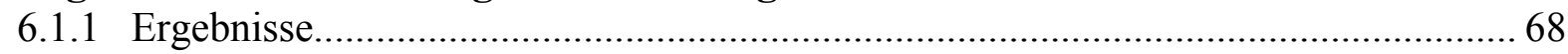

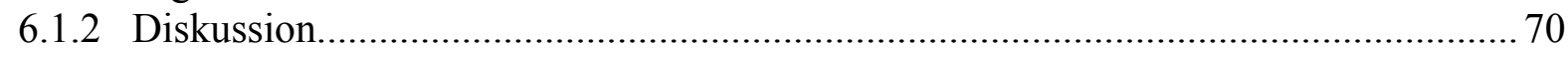

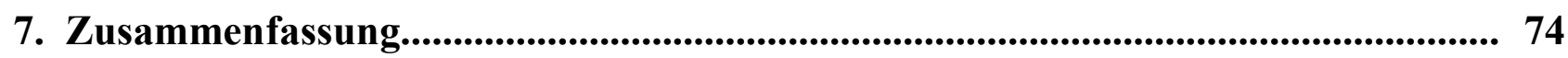

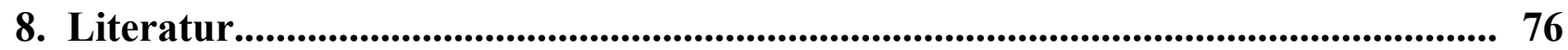

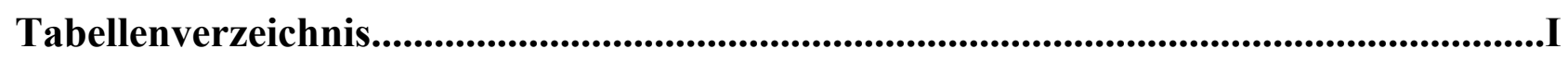

Abbildungsverzeichnis........................................................................................................................II

Abkürzungsverzeichnis.......................................................................................................................III

Anhang 1: Verzeichnis der Pflanzensippen.....................................................................

Anhang 2: Plotkoordinaten......................................................................................... XVII 


\section{Einleitung und Fragestellungen}

Der Begriff Biodiversität, der zu Beginn der 1980er Jahre geprägt wurde, findet weltweit mehr und mehr Eingang in ökologische, politische und sozio-ökonomische Debatten. Dabei ist der genaue Ursprung des Begriffes nicht bekannt und auch eine einheitliche Definition ließ lange auf sich warten. Ganz allgemein kann Biodiversität als biologische Vielfalt oder die Vielfalt des Lebens bezeichnet werden (Новонм 2000: 3). Seit der Konferenz der Vereinten Nationen für Umwelt und Entwicklung in Rio de Janeiro 1992 wird biologische Vielfalt meist folgendermaßen definiert: „die Variabilität unter lebenden Organismen jeglicher Herkunft [...] und die ökologischen Komplexe, zu denen sie gehören; dies umfasst die Vielfalt innerhalb der Arten und zwischen den Arten und die Vielfalt der Ökosysteme“ (Art. 2, in Der Bundesumweltminister o. J., 24).

Biodiversität stellt somit einen Sammelbegriff dar, der sich in drei Ebenen unterteilen lässt (Crawley 1997: 595, Primack 1997: 27 f.):

1. die genetische Vielfalt, auch als intraspezifische Diversität bezeichnet,

2. die Vielfalt der Arten, auch als interspezifische Diversität bezeichnet,

3. die Vielfalt der Artengemeinschaften und Ökosysteme.

Fragen der Biodiversität waren lange Zeit Teil rein ökologisch motivierter Wissenschaft. Inzwischen werden ihre Gefährdung und ihr fortschreitender Rückgang aber von Vertretern verschiedener wissenschaftlicher Disziplinen sowie sozialer und politischer Interessengruppen (teils unterschiedlich interpretiert) diskutiert (Pullin 2002: 6, LÉvêQue \& MounOLOu 2003: 5). So finden sich interdisziplinäre Forschungsansätze, die sich mit der Biodiversität beschäftigen nicht mehr nur in der Ökologie, sondern auch in der Soziologie und der Ökonomie.

In der Ökologie beruht ein großer Teil der Biodiversitätsforschung auf der Annahme, dass die Artenvielfalt einen wichtigen Einfluss auf die Stabilität und die Funktion eines Ökosystems hat (Lepš 2005). Um die Vielfalt der Arten feiner einzuteilen, zu messen und vergleichbar zu machen, sind mehrere Diversitäts-Indizes entwickelt worden. Es galt eine Quantifizierung der Diversität zu ermöglichen. $\mathrm{Zu}$ diesen Indizes zählen zum Beispiel der Shannon / Wiener Index, als Maß für die Mannigfaltigkeit der Arten sowie die Eveness, welche ein Maß für die Gleichverteilung von Arten darstellt (DierschKe 1994).

Die Messung von Biodiversität ist immer maßstabsabhängig (CRAwLEY 1997: 595). Die Artenzahl kann keine Information zum Artenreichtum eines Gebietes liefern, wenn die Flächengröße nicht berücksichtigt wird, auf der sie bestimmt wurde. Artenzahlen in Abhängigkeit zur Flächengröße bezeichne ich als Artendichten. Aufgrund langjähriger Feldarbeit vieler Pflanzensoziologen sind die Artendichten verschiedener Pflanzengesellschaften auf verschiedenen Flächengrößen sehr gut bekannt (DiEKMANN et al. 2007). Allerdings mangelt es oft an einheitlichen Aufnahmemethoden, um diese Artendichten miteinander vergleichen zu können (DENGLER 2003).

Ein weiteres Manko ist die Beschränkung der Aufnahmeflächen auf visuell homogene Pflanzenbestände. Für viele Pflanzensoziologen stellt das Homogenitätskriterium eines der wesentlichen Aspekte bei der Auswahl von Aufnahmeflächen dar (z. B. Dierssen 1990: 15, Dierschke 1994: 151, Glavac 1996: 84 f.). Palmer \& White (1994) führen jedoch an, dass praktikable und objektive Verfahren zur Ermittlung von Homogenität fehlen. Worauf sich diese Homogenität beziehen und in welcher Intensität sie vorhanden sein muss, damit ein Vegetationsabschnitt als homogen genug empfunden werden kann, um die Abgrenzung einer 
Aufnahmefläche zu rechtfertigen, ist demnach unklar. DierschKe (1994: 139) merkt an, dass die Betrachtung von Homogenität nicht frei von subjektiven Kriterien sei und von den Anforderungen und Ansichten des Bearbeiters abhänge.

Aufgrund des Mangels an einheitlichen Aufnahmemethoden und der Beschränkung auf homogene Bestände sind durchschnittliche Artenzahlen auf verschiedenen räumlichen Skalenebenen bezogen auf eine Region weniger gut bekannt. Новонм (2000: 94) gibt für offene Landschaften Mitteleuropas eine durchschnittliche Artenzahl von zehn Arten pro Quadratmeter an. Diese Angabe umfasst Gefäßpflanzen, Moose und Flechten, beruht aber auf der Erhebung von Artendichten in homogenen Beständen. Crawley \& Harral (2001) haben in einer Studie in Südengland für die gleiche Flächengröße eine durchschnittliche Artenzahl für Gefäßpflanzen von 6,38 ermittelt. Flechten und Moose wurden hier vernachlässigt, dabei sind sie oft ein wichtiger Bestandteil lokaler Diversität und können bis zu einem Viertel der Gesamtartenzahl aus machen (PHARO et al. 1999).

In der aktuellen Forschung zur Dichte von Pflanzenarten zeigen sich also deutliche Lücken bei der Erhebung von Artendichten in kontinuierlicher Vegetation einerseits und bezüglich der Berücksichtigung von Moosen und Flechten andererseits. Um diese Lücken zu schließen bedarf es der Untersuchung der Artendichten von Gefäßpflanzen, Moosen und Flechten auf absolut zufallsverteilten Stichproben. Daher werde ich im Rahmen dieser Diplomarbeit auf folgende Frage eingehen:

Wie hoch sind die durchschnittlichen Artenzahlen auf unterschiedlichen Flächengrößen insgesamt und differenziert nach Artengruppen und floristischem Status bei einer zufälligen Stichprobenverteilung?

Von großem Interesse in diesem Zusammenhang ist außerdem die Untersuchung von Arten-ArealBeziehungen, welche zu den ältesten und meist untersuchten Beziehungen in der Ökologie (Lomolino 2000, TuRner \& Tıørve 2005) gehören und als ökologische Gesetzmäßigkeit gelten (SCHeINER 2003).

In der Pflanzensoziologie wurden Arten-Areal-Beziehungen intensiv untersucht, da man glaubte mit Hilfe von Arten-Areal-Kurven das methodologische Minimumareal einer Pflanzengesellschaft bestimmen zu können. Eine Pflanzengesellschaft im Sinne von Braun-Blanquet (1951) stellt eine regelhafte, typisierbare Vergesellschaftung von Pflanzen dar, die sich jeweils durch bestimmte Arten von anderen Vegetationstypen unterscheiden. Das Minimumareal entspricht der kleinsten Flächengröße, auf der die Artenzusammensetzung der betrachteten Pflanzengesellschaft adäquat repräsentiert wird (MülLer-Dombois \& Ellenberg 1974: 47). Diese Flächengröße definiert ElLenBerg (1956: 18) als diejenige, „bei der die anfangs steil ansteigende Kurve in den fast waagerecht verlaufenden Ast umbiegt“. Das Minimumareal stellt demnach eine Flächengröße dar, auf der die Artenzunahme der untersuchten Pflanzengesellschaft nahezu gesättigt ist. Dieses Konzept ist viel diskutiert und kritisiert worden (vgl. Dengler 2003: 69 ff.).

Dabei spielen Arten-Areal-Beziehungen in vielen fundamentalen Forschungen eine wichtige Rolle. Dazu zählen Häufigkeitsverteilungen von Arten (May 1975, Harte et al. 1999, Pueyo 2006), Vergleiche des Artenreichtums unterschiedlicher Gebiete (ChIARUCCI et al. 2006) oder die Untersuchungen zu den Artendichten bestimmter Artengruppen oder Landschaftstypen (CRAWLEY \& Harral 2001, Chiarucci et al. 2006). All diese Forschungen finden außerdem Anwendung in anderen Disziplinen wie zum Beispiel im Naturschutz (Desmet \& Cowling 2004).

Die wohl populärste Funktion zum Zusammenhang von Artenzahl und Flächengröße hat ArrhENIUS schon im Jahr 1921 postuliert. Er nahm an, dass eine Potenzfunktion diesen Zusammenhang am 
besten beschreibt. In den folgenden Jahrzehnten sind weitere Formeln aufgestellt worden, die diesen Zusammenhang insgesamt oder zumindest für bestimmte Vegetationstypen besser beschreiben sollten. Neben der Potenzfunktion von Arrhenius findet vor allem die Logarithmusfunktion von Gleason $(1922,1925)$ viel Beachtung. Neben diesen beiden wird vor allem linearen Funktionen (vgl. Connor \& McCoy 1979) und Sättigungsfunktionen, wie z. B. der Michaelis-Menten-Funktion eine gute Approximation der Artenzahlen unterstellt (Sснмітт 1999).

Einen Vergleich verschiedener mathematischer Funktionen für den Verlauf von Arten-ArealKurven bieten zum Beispiel He \& Legendre (1996), Tuørve (2003) und Scheiner (2003). Allerdings bedienen sich viele Autoren vorhandener Literaturdaten oder führen ihre Untersuchungen von vornherein rein theoretisch durch, was zum Teil daran liegen mag, dass Artendichten nicht für beliebig viele Flächengrößen zur Verfügung stehen. Im Rahmen der vorliegenden Arbeit wurden auf 50 Plots die Artenzahlen auf sieben verschiedenen Flächengrößen erhoben. Mit diesem Datenset strebe ich eine Evaluation verschiedener Kurven anhand von speziellen Gütekriterien an. Meine zweite Fragestellung lautet diesbezüglich:

Welche Funktion beschreibt den Zusammenhang von Artenzahl und Flächengröße am besten?

Die Steigung der Kurven enthält dabei die wesentliche Information einer Arten-Areal-Kurve, da sie den Anstieg der Arten zwischen zwei verschieden großen Flächen wiedergibt. Während man früher davon ausging, dass die Steigung als Konstante betrachtet werden kann, wird sie in Studien der letzten Jahre in Bezug zur Flächengröße oder zu bestimmten Größenbereichen der Fläche gesetzt (z. B. Crawley \& Harral 2001, Chiarucci et al. 2006). Daher möchte ich in diesem Zusammenhang noch auf folgende Frage eingehen:

Wie verändert sich die Steigung einer Arten-Areal-Kurve bei zunehmender Flächengröße für die Arten insgesamt und differenziert nach Artengruppen und Landschaftstypen?

Dabei ist der wissenschaftliche Diskurs um Arten-Areal-Beziehungen so komplex und war in den letzten Jahrzehnten Gegenstand unzähliger Publikationen, weshalb im Rahmen dieser Arbeit nur ein kurzer Einblick in die Diskussion anhand des empirischen Datensets gegeben werden kann.

Abschließend widme ich mich der Frage nach den häufigsten Arten. Die Häufigkeit einer bestimmten Art ist in Deutschland nur auf Flächen eines Messtischblatt oder eines MesstischblattQuadranten bekannt (vgl. Haeupler \& Schönfelder 1989, Benkert et al. 1996). Angaben lokaler Häufigkeiten der Gefäßpflanzen finden sich zwar in Ellenberg et al. (1991). Trotzdem fehlen in der Literatur bislang Angaben darüber, welche Pflanzenarten (Moose und Flechten inbegriffen) auf Flächengrößen kleiner als $100 \mathrm{~m}^{2}$ durchschnittlich die häufigsten sind. Daher gehe ich in meiner letzten Frage den Häufigkeiten einzelner Arten nach:

Welches sind die häufigsten Pflanzenarten im Untersuchungsgebiet? Wie sind sie ihrer Häufigkeit nach verteilt? 


\section{Das Untersuchungsgebiet}

\subsection{Lage und allgemeine Gebietsbeschreibung}

Das Untersuchungsgebiet entspricht genau dem Messtischblatt 2728 Lüneburg der Topographischen Karte (TK) im Maßstab 1:25000 herausgegeben vom Landesvermessungsamt Niedersachsen. Es liegt im norddeutschen Tiefland etwa $50 \mathrm{~km}$ südöstlich von Hamburg im Nordosten Niedersachsens, zwischen dem Nationalpark Elbtalaue und dem Wendland im Osten und dem Naturschutzpark Lüneburger Heide im Südwesten (Abb. 1). Das gesamte Gebiet erstreckt sich von $53^{\circ} 12^{\prime}$ bis $53^{\circ} 18^{\prime}$ nördlicher Länge und von $10^{\circ} 20^{\prime}$ bis $10^{\circ} 30^{\prime}$ östlicher Breite. Das entspricht für dieses Messtischblatt einer Kantenlänge sowohl in Nord-Süd- als auch in Ost-WestRichtung von ungefähr 11,25 km. Die Mittelstadt Lüneburg liegt dabei ziemlich zentral in dieser ca. $126 \mathrm{~km}^{2}$ großen Fläche und nimmt selber eine Fläche von $70,34 \mathrm{~km}^{2}$ ein.

Abbildung 1: Lage des Untersuchungsgebietes in einer Karte aus Winter \& BeCKeL (1992).

Die Landschaft besteht aus ca. $35 \%$ Wäldern, $30 \%$ Agrarfläche, $20 \%$ Siedlungsfläche, $10 \%$ Grünland und 5 \% Industrie- und Gewerbefläche. Das Gebiet wird durch den Fluss Ilmenau in zwei Hälften von fast gleicher Größe geteilt. Der Flussverlauf stellt die tiefste Linie des Gebietes dar, wobei das ganze Areal mit Höhen zwischen 15 und $50 \mathrm{~m}$ über NN relativ flach ist. Lediglich im Südosten und Osten gibt es Erhebungen mit bis zu 80 m über NN. 


\subsection{Klima}

Die Lüneburger Heide liegt großklimatisch in einer subatlantischen, gemäßigten Zone mit kühlen Wintern und milden Sommern bei ganzjährigen Niederschlägen. Richtung Osten dagegen (Wendland, Elbe) findet sich bereits subkontinentales Klima. Das Untersuchungsgebiet liegt also im Übergangsbereich und hat subatlantisch-subkontinentales Klima. Die Abbildungen 2 bis 5 zeigen Klimadiagramme für die Stadt Lüneburg (aus Müller \& Westermeier 1996). Die Klimadaten, die diesen Diagrammen zugrunde liegen sind von 1961 bis 1990 erhoben worden. Alle Angaben stellen Mittelwerte über diese Periode dar. Die Jahresmitteltemperatur beträgt in Lüneburg $8,9{ }^{\circ} \mathrm{C}$. Während es durchschnittlich 95 Eis- und Frosttage im Jahr gibt, zählen Sommertage mit mehr als $25^{\circ} \mathrm{C}$ im Schnitt 24 (Abb. 2).

Abbildung 2: Lufttemperatur in Lüneburg aus MülLER \& WestermeIER (1996).

Die relative Luftfeuchtigkeit liegt in Lüneburg im Durchschnitt um $80 \%$, im Winter etwas höher, im Sommer etwas niedriger (Abb 3).

Abbildung 3: Luftfeuchte in Lüneburg aus MüLLER \& WestermeIER (1996).

Die durchschnittliche Jahresniederschlagshöhe liegt in Lüneburg bei $611 \mathrm{~mm}$ (HAVERSATH 1997). In 
den Sommermonaten Juni, Juli und August fällt dabei der meiste Niederschlag, was auf die größere Anzahl von Tagen mit mehr als $10 \mathrm{~mm}$ Niederschlag zurückgeführt werden kann. Insgesamt fällt jährlich an etwa 130 Tagen Niederschlag (Abb 4).

Abbildung 4: Niederschlag in Lüneburg aus MüLLER \& WeSTERMEIER (1996).

Gleichzeitig ist die mittlere Bewölkung im Sommer geringer als im übrigen Jahresverlauf. Die mittlere jährliche Sonnenscheindauer beträgt ca. 1500 Stunden (Abb. 5).

Abbildung 5: Sonnenschein und Bewölkung in Lüneburg aus MülLER \& WeSTERMEIER (1996). 


\subsection{Physische Geographie und Böden}

Das Untersuchungsgebiet gehört geologisch zum Südlichen Landrücken, der Teil des Altmoränenlandes ist. Geformt wurde diese Landschaft vor allem während der Saale-Eiszeit. Innerhalb dieser gab es mehrere Eisvorstöße, die verantwortlich sind für die flachen Ebenen der Grundmoränen sowie die hügeligen Endmoränenzüge in Norddeutschland (SEMmel 1984: 123, Henningsen \& Katzung 2002: 177 ff., Ротт 1999: 10 ff.).

Abbildung 6: Das Untersuchungsgebiet im Nordosten Niedersachsens aus TOP50 Niedersachsen/Bremen (LANDESvermessung Und Geobasisinformation Niedersachsen 2003).

Nach Meynen et al. (1962) liegt das Untersuchungsgebiet in der naturräumlichen Haupteinheit Lüneburger Heide. Diese lässt sich in die Einheiten Hohe Heide, Südheide, Ostheide, Luheheide und Uelzener Becken unterteilen. Der größte Teil des Untersuchungsgebietes liegt demnach in der Luheheide. Nur ein auf die Fläche bezogen geringer Teil östlich des Elbe-Seiten-Kanals wird der Ostheide zugesprochen. Geologisch betrachtet besteht die Luheheide aus Grundmoränenplatten, die im Süden, Osten und Westen von Endmoränenzügen eingeschlossen sind und im Norden zum Urstromtal der Elbe abfallen (VÖLKSEN 1984: 11). Die Endmoränenzüge der Lüneburger Heide sind neuesten Untersuchungen zufolge bereits im Drenthe-Stadium entstanden und während des WartheStadiums noch einmal überformt worden (Ротт 1999: 18). Nach HenNingsen \& Katzung (2002) herrschen in der weiteren Umgebung Lüneburgs Bildungen des Warthe-Stadiums vor. Einen Überblick über die Diskussion zur Abgrenzung der Warthe-eiszeitlichen Moränen im Norddeutschen Tiefland gibt Semmel (1984: 127 f.).

Als Besonderheit des Gebietes gelten die Salzsteinschichten. Sie entstehen, wenn Meersalz nach Verdampfen des Wassers in Tiefen von mehreren tausend Metern absinkt und dann bei starkem Druck und hoher Temperatur plastisch verformbar wird. So können sich die Salzschichten durch tektonische Bewegungen der Erdkruste in Form von Salzstöcken oder -diapiren der Erdoberfläche nähern oder diese sogar durchbrechen. Ein bekanntes Beispiel hierfür ist der Kalkberg in Lüneburg, der nahezu den Mittelpunkt des berücksichtigten Untersuchungsgebietes darstellt. Der Salzstock 
unter Lüneburg ist ca. 1500 m mächtig und liegt im Durchschnitt etwa 40 m unter der Oberfläche (DRIESNER 1988).

Im norddeutschen Tiefland kommen fast nur quartäre Gesteine vor. Da im Quartär Festgesteine fast völlig fehlen (SEmmel 1984: 20), erklären sich die zumeist geringen Reliefunterschiede (HenNingSEN \& KATZung 2002: 166) in dieser Region durch die Decke quartärer Lockersedimente. Diese setzen sich aus fluviatilen Kiesen, Solifluktionsschuttmassen sowie Windsedimenten (Flugsand und Löss) zusammen.

Geomorphologisch gehören die Böden des Untersuchungsgebietes hauptsächlich zu Grundmoränen der Geestlandschaft. Lediglich im Norden fällt die Landschaft $\mathrm{zu}$ den Talsandniederungen und Urstromtälern der Elbe ab. Während die Ilmenau eines der wenigen Landschaftselemente mit Talsedimenten (Auensande, Abschlämmmassen) darstellt, herrschen ansonsten sandige Böden mit fluviatilen und glazifluviatilen Sedimenten (Geschiebemergel) vor. Daraus ergibt sich an vielen Stellen ein Mosaik verschiedener Bodenarten (VöLKSEN 1984: 11). Die vorherrschenden Bodentypen sind die Podsol-Braunerde und Pseudogley mit schwach lehmigen meist pleistozänen Sanden. Außerdem kommen häufig Podsole oder podsolierte Bodentypen mit z. T. steinigen oder kiesigen Sanden vor. Besonders im Norden und entlang der Ilmenau im grundwassernahen, ebenen Gelände finden sich Gley und Podsol-Gley mit fluviatilen Sanden. Im Südwesten des Untersuchungsgebiets gibt es außerdem einen schmalen Streifen Niedermoor mit Niedermoortorfen über fluviatilen Sedimenten.

\subsection{Nutzungs- und Kulturgeschichte}

Die landschaftsprägende Flächennutzung in und um Lüneburg war bis zum Anfang des 20. Jh. die Heidewirtschaft. Durch sie entstand eine Kulturlandschaft, die sich in hunderten von Jahren entwickelt hat und heute nur noch auf ca. 3100 ha im Naturschutzgebiet Lüneburger Heide erhalten ist (Von DER LANCKEN 1997). Durch frühzeitige Schutzbemühungen dieser Region finden sich hier im Gegensatz zu vielen anderen Regionen auch noch viele archäologische Funde. Aus Mangel an schriftlichen Quellen stellen sie die einzige Möglichkeit dar sich von vorgeschichtlichen Zeiten ein Bild zu machen (LÜTKEPOHL \& HANSTEIN 1997).

Heidevegetation war in dieser Phase noch nicht verbreitet, wie Boess (1997) anführt. Der Mensch hat aber bereits in dieser frühen Zeit begonnen die Entwicklung der Wälder zu beeinflussen. Laut VöLKSEN (1984: 12) lassen sich erste Spuren menschlicher Besiedelung in dieser Region 50.000 Jahre zurückverfolgen. In der Nähe vom nur $12 \mathrm{~km}$ vom Untersuchungsgebiet entfernt liegenden Ort Hörpel wurde sogar ein Faustkeil aus dem frühen Mittelpaläolithikum, welches vor 200.000 Jahren begann, gefunden (LütKepohl \& Hanstein 1997). Reinhardt (1982) gibt für den heutigen Landkreis Lüneburg (1323 km²) 41 Hünengräber aus der jüngeren Steinzeit (ca. 5000 v. Chr.) an.

Mit Beginn des Neolithikums (4500-1700 v. Chr.) nahmen die Aktivitäten des Menschen deutlich zu. Es muss stellenweise schon dichtere Besiedelung gegeben haben, da sich bereits viele Denkmäler dieser Zeit im Raum Lüneburg finden (REINHARDT 1982). Waldackerbau und Viehzucht sind entstanden und veränderten die Landschaft durch einen Rückgang der Wälder. Diese haben sich inzwischen noch stärker zu Eichen- und Buchenmischwäldern entwickelt. Mit Hilfe von Pollenanalysen konnte die Zwergstrauchheide als wesentlicher Bestandteil der Vegetation in der Bronzezeit (2200-800 v. Chr.) nachgewiesen werden. Zur Eisenzeit nahm die Zwergstrauchheide 
bereits nennenswerte Teile Norddeutschlands ein (VöLKSEN 1984: 13). Heidevegetation aber kann sich nur da entwickeln, wo kein Wald ist. Große Waldflächen sind also schon damals abgeholzt worden um (besonders im Umkreis von Siedlungen) den Holzbedarf der Menschen zu decken und Weidefläche für die Viehhaltung zu schaffen. Trotzdem prägten bis zum Beginn des frühen Mittelalters ausgedehnte Laubmischwälder die Landschaft. Das änderte sich im Mittelalter rapide.

In den ersten Jahrhunderten n. Chr. war der Wald durch menschliche Aktivitäten schon stark gelichtet und Heideflächen weit verbreitet. Im Lüneburger Raum hat neben dem allgemeinen Bevölkerungszuwachs auch die Salzgewinnung zu hohem Holzbedarf geführt. Aber auch in vielen anderen Wirtschaftszweigen war Holz das wichtigste Gut, so dass VöLKSEN (1984: 15) anführt, dass es in der europäischen Geschichte nie eine Zeit gegeben hat, in der die Existenz des Wirtschaftssystems so ausschließlich vom Holz abhing, wie es im Mittelalter der Fall war.

Im 18. Jh. erreichte die Ausdehnung der Heideflächen ihren Höhepunkt mit einem Verhältnis von Wald zu Heide von stellenweise 1 : 400. Ende des 18. Jh., Anfang des 19. Jh. kam die Heidewirtschaft in eine ökonomische und ökologische Krise. Daraufhin wurden weite Teil der Heidefläche entweder intensiver genutzt oder aufgeforstet. Während 1776 ca. zwei Drittel der Fläche um Lüneburg Heidefläche war, betrug der Anteil 1901 weniger als ein Drittel. Weitere 50 Jahre später gab es nur noch wenige isolierte Vorkommen. Gleichzeitig wuchsen Grundfläche und Einwohnerzahl der Stadt Lüneburg.

Im November 2006 betrug die Bevölkerungsdichte in Lüneburg 1024 Einwohner pro km², während die des Landkreises Lüneburg zu diesem Zeitpunkt 132,8 Einwohner pro $\mathrm{km}^{2}$ betrug. Diese Zahlen liegen unter dem Durchschnitt für Niedersachsen mit einer Bevölkerungsdichte von 168 Einwohnern pro Quadratkilometer (Stand: 31.12.2005). Für das Untersuchungsgebiet kann man eine Dichte von ca. 840 Einwohnern pro Quadratkilometer berechnen. Diese Berechnung beruht auf den Einwohnerzahlen der in dem Messtischblatt angesiedelten Ortschaften. Die Einwohnerzahlen habe ich vom Niedersächsischen Landesamt für Statistik übernommen (NIEDERSÄCHSISCHES LANDESAMT FÜR STATISTIK 2007).

\subsection{Vegetation und Flora}

Nach dem Rückzug des Inlandeises in Norddeutschland (vor ca. 10.000 Jahren) herrschte zunächst Tundrenvegetation mit Moosen, Gräsern und Sträuchern vor. Dies änderte sich erst ca. 7500 Jahre v. u. Z. durch eingewanderte Baumarten. Hierzu zählten zunächst Pinus sylvestris (Wald-Kiefer) und Betula spec. (Birke), die sich als bestandsbildende Baumarten durchsetzten (Ротт 1999: 36, Boess 1997). VöLKSEN (1984: 12) erweitert das Artenspektrum um Populus tremula (Zitterpappel) und Salix caprea (Salweide) und bezeichnet diese Epoche als Birkenzeit. Im Atlantikum, welches das klimatische Optimum des Holozäns darstellte, kamen viele waldbildende Baumarten wie Ulmus spec. (Ulme), Quercus spec. (Eiche), Fraxinus spec. (Esche), Picea spec. (Fichte) und Tilia spec. (Linde) hinzu. Erst 2000 Jahre später, im Subboreal wurde die Baumartenvielfalt durch Abies spec. (Tanne) sowie Carpinus betulus (Hainbuche) und Fagus sylvatica (Rotbuche) ergänzt (HaversaTH 1997: 44). Die landwirtschaftlichen Aktivitäten des Menschen haben in der Folgezeit einen Rückgang der Waldflächen und die Entstehung von Heideflächen bewirkt. Insgesamt hat Niedersachsen derzeit einen Waldanteil von 24,3\%, wobei Nadelholzbewuchs überwiegt (Niedersächsisches Ministerium FÜr DEN LÄNDLIChen Raum, ERnÄHrung, LANDwirtschaft und 
Verbraucherschutz 2004). Das Ergebnis ist eine einzigartige Kulturlandschaft, die jedoch nicht der natürlichen Vegetation in dieser Region entspricht.

In Norddeutschland kämen als potenziell natürliche Vegetation mesophytische, sommergrüne Laubwälder und Nadel-Laubwälder vor. Im Untersuchungsgebiet entsprächen hygrophile BirkenStieleichenwälder sowie Eichen-Buchenwälder der natürlichen Vegetation (BoHN \& NeuHäUsL 2000, BoHn et al. 2000, 2003, 2004).

Die Birken-Stieleichenwälder (nach Ellenberg (1996) Betulo-Quercetum typicum) zeichnen sich durch eine Baumschicht von Quercus robur (Stieleiche), Betula pubescens (Moorbirke) und Betula pendula (Hängebirke) aus, die nur gelegentlich von Fagus sylvatica, Populus tremula sowie Sorbus aucuparia (Eberesche), welche auch in der gering entwickelten Strauchschicht zu finden ist, ergänzt wird. Häufige Arten in der Krautschicht sind neben anderen z. B. Deschampsia flexuosa (Drahtschmiele), Dryopteris carthusiana (Dorniger Wurmfarn), Carex pilulifera (Pillen-Segge), Holcus mollis (Weiches Honiggras), Lonicera periclymenum (Deutsches Geißblatt), Ceratocapnos claviculata (Europäischer Rankenlerchensporn), Vaccinium myrtillus (Heidelbeere), Rubus idaeus (Himbeere), Trientalis europaea (Europäischer Siebenstern), Oxalis acetosella (Wald-Sauerklee), Lysimachia vulgaris (Gewöhnlicher Gilbweiderich) und Juncus effusus (Flatter-Binse). Der im Kap. 2.2 beschriebenen Geologie des Gebietes entsprechend sind die typischen geologischen Unterlagen dieses Vegetationstyps unter anderem Grund- und Endmoränen des Warthe-Stadiums (BoHN et al. 2000, 2003, 2004).

Die Eichen-Buchenwälder (nach Ellenberg (1996) Fago-Quercetum typicum) als natürliche Vegetation in dieser Region haben als dominante Baumart eigentlich nur Fagus sylvatica mit nur gelegentlich eingestreut vorkommender Quercus robur vorzuweisen. Während in der Strauchschicht neben Sorbus aucuparia und Frangula alnus (Faulbaum) Ilex aquifolium (Stechpalme) in z. T. dichten Beständen steht, ist die Krautschicht ähnlich besiedelt wie die der Birken-Stieleichenwälder. Auch hier entsprechen die Angaben zur Geomorphologie und Geologie sehr gut dem Untersuchungsgebiet: Tiefland mit sandigem Untergrund und Grund- und Endmoränen der Saale-Eiszeit (BoHn et al. 2000, 2003, 2004).

Die bereits erwähnte klimatische Übergangslage des Untersuchungsgebietes fördert eine interessante Florenzusammensetzung. Man findet hier sowohl Arten subkontinentalen Verbreitungsschwerpunktes (z. B. Hieracium caespitosum (Wiesen-Habichtskraut) und Maianthemum bifolium (Zweiblättrige Schattenblume) sowie viele Flechtenarten) als auch solche mit atlantisch-subatlantischem Areal (z. B. Bromus sterilis (Taube Trespe), Ilex aquifolium und Erica tetralix (Glocken-Heide)). Laut Ротт (1999: 57) finden sich jedoch auch südliche Arten, die entlang der Elbe nach Norden wandern konnten. Dazu gehören unter anderem Allium schoenoprasum (Schnittlauch) und Rumex thyrsiflorus (Rispen-Sauerampfer). Aber auch nördliche boreale Elemente, beispielsweise Trientalis europaea und Vaccinium myrtillus, dringen in den Untersuchungsraum vor. Außerdem finden sich auch atlantische Arten. Als besonders erwähnenswert erscheint mir hier Ceratocapnos claviculata, da seine Kontinentalität in ElLENBERG et al. (1991) als euozeanisch eingestuft wird.

Insgesamt sind für dieses Gebiet 797 Gefäßpflanzenarten erfasst worden (HAEUPLER \& SchÖNFELDER 1989: 35), von denen 145 auf der Roten Liste Niedersachsens stehen (GARVE 1994: 28). Durchschnittlich beherbergt ein Messtischblatt in Deutschland 630 Gefäßpflanzen, so dass sich für das Untersuchungsgebiet, welches einem Messtischblatt entspricht, eine hohe Artendichte feststellen lässt. GARVE (1994) ordnet Nordostniedersachsen eine Vielfalt von 1471 Gefäßpflanzen zu, bezieht sich dabei aber auf eine fast $4000 \mathrm{~km}^{2}$ große Fläche. Bezüglich der gefährdeten Arten 
fällt auf, dass die beiden Quadranten östlich der Ilmenau im Durchschnitt 32 gefährdete Arten aufweisen, während die im Westen durchschnittlich 76 gefährdete Arten beherbergen. Dies liegt vermutlich vor allem daran, dass das Naturschutzgebiet Lüneburger Kalkberg im Westen liegt und dabei die Nord-Süd-Grenze der westlichen Quadranten überschneidet. In diesem Gebiet konnte bereits Horst (1983) nach der damaligen Roten Liste von Niedersachsen (HAEupLer et al. 1983) 28 gefährdete Gefäßpflanzenarten feststellen. Derzeit gibt es nach dem aktuellen Atlas der gefährdeten Farn- und Blütenpflanzen in Niedersachsen und Bremen (GARve 2004) sogar rund 40 gefährdete Gefäßpflanzenarten (Dengler \& Boch 2006). 


\section{Die Methoden}

Im folgenden sollen die dieser Arbeit zugrunde liegenden Methoden beschrieben werden. Dabei wird zunächst die die Vorgehensweise beschrieben, mit der die Aufnahmeflächen im Untersuchungsgebiet ausgewählt wurden, wonach die Anzahl und Anordnung der Aufnahmeflächen innerhalb eines Plots beschrieben wird. Anschließend gibt es eine Übersicht über die im Gelände erhobenen Daten.

\subsection{Vegetationskundliche Methoden}

\subsubsection{Auswahl der Plots}

Die Frage nach „der einen richtigen“ Prozedur bei der Auswahl der Aufnahmeflächen (Stichprobenverteilung) ist bislang nicht einheitlich $\mathrm{zu}$ beantworten und der Fragestellung des Experiments anzupassen. Es gibt hierbei verschiedene Vorgehensweisen (vgl. WILDI 1986: 33ff., Dierschke 1994: 150, Glavac 1996: 78 ff.), die alle mit ihren eigenen Vor- und Nachteilen behaftet sind (vgl. Dengler 2003: 122 ff.). Die Reproduzierbarkeit dieser Arbeit stellt eine wesentliche Anforderung an die Methodik dar. Daher galt es alle Kriterien zu vermeiden, die - wie eben dieses Homogenitätskriterium - stark subjektiv sein müssen. Die Ergebnisse dieser Arbeit sollen außerdem repräsentativ für die mitteleuropäische Kulturlandschaft sein, was durch eine Stratifizierung der Gesamtfläche in Landschaftstypen (wie Wald, Ackerfläche etc.) unterbunden worden wäre. Außerdem wäre eine genaue Bestimmung der Flächenanteile selbst mit einem Geoinformationssystem schwer, aufwendig und diesen Aufwand nicht Wert gewesen. Daher habe ich mich für eine absolut zufällige Verteilung der Aufnahmeflächen entschieden. Ein wesentlicher Nachteil dieses Verfahrens ist die Tatsache, dass seltene Vegetationstypen bzw. Pflanzengesellschaften nicht erfasst werden. Bei entsprechend ungünstiger Verteilung können auch häufige Vegetationstypen relativ zu ihrem Flächenanteil unterrepräsentiert sein. Um dies zu vermeiden, muss die Anzahl der Aufnahmeflächen möglichst hoch sein. Je höher die Stichprobenanzahl desto wahrscheinlicher ist eine Gleichberechtigung der Flächeneinheiten zu erreichen.

Die Koordinaten des südwestlichen Punktes meiner Plots habe ich mit Hilfe des Zufallszahlengenerators des Programmes Microsoft Excel 2000 erzeugt. Dafür habe ich in Excel eine der Anzahl meiner Plots entsprechende Menge von Zahlen zwischen 0 und 1 ausgeben lassen. Diese habe ich dann mit der Differenz des niedrigsten und höchsten Punktes des Rechts- bzw. Hochwertes meines Messtischblattes multipliziert. Da sich mit einem geodätische Datum mit Bogenminuten und Bogensekunden nicht gut rechnen lässt, habe ich die Grenzkoordinaten des Untersuchungsgebietes in Gauß-Krüger-Koordinaten umgerechnet. Dabei mussten $10 \mathrm{~m}$ Puffer am nördlichen sowie östlichen Rand des Untersuchungsgebietes berücksichtigt werden, damit eine eingenordete, nach Osten aufgespannte quadratische Aufnahmefläche nicht über die Grenzen des

Messtischblattes hinausgeht. Hier war eine Umrechnung in Gauß-Krüger Koordinaten ebenfalls vorteilhaft, da es sich um ein metrisches System handelt. Die Gauß-Krüger-Koordinaten der Eckpunkte des untersuchten Messtischblattes habe ich durch Umrechnung der geographischen Koordinaten erhalten. Das Ergebnis der Multiplikation habe ich dann jeweils auf den westlichsten 
Rechts- bzw. den südlichsten Hochwert addiert. Auf diese Weise habe ich mir 90 Koordinaten für zunächst 50 Plots generieren lassen. Den Puffer von 40 Punkten habe ich erstellt, damit ich unzugängliche Aufnahmeflächen ersetzen oder bei unerwartet schnellem Fortschritt zusätzliche Plots bearbeiten konnte. Die ersten 50 Plots habe ich unabhängig von ihrer Nummer aufgesucht. Konnte ich einen von den ersten 50 Plots nicht aufnehmen, habe ich den Plot als Ersatz aufgenommen, der als nächster in der Liste dem gleichen Landschaftstyp entsprach. Dadurch wurde gewährleistet, dass alle Landschaftstypen entsprechend ihrer ursprünglichen Stichprobenanzahl berücksichtigt wurden, was wichtig war um die repräsentative Wiedergabe der Flächenanteile der Landschaftstypen zu erhalten.

Die Plotkoordinaten der Aufnahmeflächen habe ich mit einem GPS-Empfänger ${ }^{1}$ aufgesucht. Leider verfügen auch moderne GPS-Geräte nicht über eine Genauigkeit, die es ermöglicht Koordinaten auf einen Meter genau aufzusuchen. Eine genaue Positionsbestimmung im Gelände war daher ausgeschlossen. Um eine konstistente und objektive Vorgehensweise zu schaffen, habe ich immer exakt die Stelle, an der sich laut GPS-Empfänger zum ersten mal eine Entfernung zur Plotkoordinate von $15 \mathrm{~m}$ einstellte, als südwestliche Ecke der Aufnahmefläche markiert. Diese Distanz stellte den besten Kompromiss dar, da einerseits eine möglichst geringe Verfälschung der Plotkoordinaten wünschenswert war, aber besonders in Wäldern der Empfang eines GPS-Signal als sehr schlecht ermittlet wurde. Bei dieser Vorgehensweise musste ich der Richtungsvorgabe des GPS-Empfängers folgen und auf die Entfernungsangabe achten, wodurch ich mich weder bewusst noch unbewusst von der Umwelt beeinflussen lassen konnte. Dies half gleichzeitig Objektivität zu wahren.

Es war davon auszugehen, dass einige der Koordinaten zu schwer erreichbaren Flächen führen würden. Als schwer erreichbar habe ich dabei vor allem Gewässer, Verkehrsflächen, Sperrgebiete und Gebäudedächer betrachtet. Um über ein konsistentes Schema im Umgang mit diesen Flächen zu verfügen, habe ich folgende Herangehensweisen festgelegt:

1. Gewässer: Je nach Wassertiefe wurde gekeschert oder nach Vegetation getaucht.

2. Verkehrsflächen: So fern möglich, ohne den Verkehr zu behindern oder sich selbst zu gefährden, konnte auch die Vegetation an und auf Straßen untersucht werden.

3. Sperrgebiete: Bei militärisch oder gewerblich genutzten Flächen konnte eine Genehmigung eingeholt werden.

4. Hausdächer: Bei Flachdachhäusern wurden die Bewohner um eine Erlaubnis gebeten, die Vegetation ihres Hausdaches untersuchen zu dürfen. Die Flora aller anderen Dächer konnten visuell per Fernglas bis zu dem präzisesten möglichen nomenklatorischen Niveau bestimmt werden.

Allgemein habe ich versucht möglichst alle Plots mit allen Teilflächen aufzunehmen, auch wenn ich dabei nicht alle Individuen bis zur Art bestimmen oder alle Teilflächen vollständig erreichen konnte.

1 Als GPS-Empfänger kam ein Garmin eTrex Venture zum Einsatz. 


\subsubsection{Aufnahmedesign}

Untersuchungen zu Artenzahl-Areal-Beziehungen erfordern ein Versuchsdesign, welches möglichst viele verschiedene Flächengrößen berücksichtigt. Dierschke (1994: 139) schlägt hierzu die Einflächenmethode vor, bei der die kleinere Fläche jeweils in der nächstgrößeren liegt. Wie DoLNIK (2003: 29) herausstellt, ist eine stete Verdoppelung der Flächengröße dabei nicht zweckmäßig. Des Weiteren merkt BARKMAN (1989) an, dass bei Biodiversitäts-Untersuchungen eine höhere Anzahl der kleineren Flächen notwendig sei, weil die Variation der Artenzahl bei kleineren Flächen größer ist als bei Größeren. Deshalb schlägt er vor die Anzahl dieser durch zufällig in die Aufnahmefläche (Plot) eingestreute kleine Flächen auf 50 oder sogar 100 zu erhöhen. Dierschke (1994: 140) hält bei der Erhebung der Artendichte auf kleinen Flächen die Erfassung mehrerer voneinander unabhängiger Flächen (5-10) für sinnvoll. Dadurch können Fehler, die bei der Aufnahme der kleineren Flächen gemacht werden, nicht in die nächstgrößeren Flächen übernommen werden.

Eine statistisch genaue Analyse zur notwendigen Anzahl von Replikaten bestimmter Flächengrößen erfolgt bei Dengler (2006) am Beispiel verschiedener Trockenrasengesellschaften. Er stellt heraus, dass der Variationskoeffizient der Artenzahl mit zunehmender Flächengröße abnimmt. Diesen Zusammenhang kann man nutzen, um die notwendige Anzahl von Wiederholungen der Artenzahlerhebungen für bestimmte Flächengrößen zu ermitteln. Dabei kommt es sowohl auf den vorausgesetzten Standardfehler als auch den Vegetationstyp oder die Pflanzengesellschaft an. Über die untersuchten Trockenrasengesellschaften gemittelt ergeben sich daraus ein Replikat für die größte Fläche, drei Replikate für ein Vielfaches der Startfläche von 0,01, bereits acht für ein Vielfaches von 0,0001 und 24 Replikate bei einem Vielfachen von 0,000001. In der Annahme, dass die im Untersuchungsgebiet anteilig wesentlichen Landschaftstypen (Agrarflächen und Wälder) kleinräumig nicht so variabel und artenreich sind, wie Trockenrasengesellschaften, und um trotz der eingeschränkten Zeit von nur einer Vegetationsperiode genug Aufnahmen für eine statistisch fundierte Auswertung machen zu können, habe ich die Anzahl der Replikate reduziert. Auf die von DierschKe (1994) vorgeschlagene zufällige Verteilung kleinerer Aufnahmeflächen außerhalb und unabhängig von den größeren Aufnahmeflächen habe ich aus zeitlichen Gründen verzichtet. Berücksichtigt wurden folgende Flächengrößen, wobei alle außer der größten Aufnahmefläche mit je vier Replikaten berücksichtigt wurden: $0,0001 \mathrm{~m}^{2}, 0,0009 \mathrm{~m}^{2}, 0,01 \mathrm{~m}^{2}, 0,09 \mathrm{~m}^{2}, 1 \mathrm{~m}^{2}, 9 \mathrm{~m}^{2}$, $100 \mathrm{~m}^{2}$. Damit erhalte ich eine Erweiterung der Flächengrößen um jeweils fast eine Zehnerpotenz. Die Spanne von der kleinsten zur größten Flächengröße ist dabei groß, während die Anzahl berücksichtigter Flächengrößen zugunsten der Bearbeitungszeit eher einen Kompromiss darstellt.

Ich werde mich bei der Beschreibung der Methoden des öfteren auf diese unterschiedlichen Flächengrößen beziehen. Der Einfachheit benenne ich diese Aufnahmeflächen im folgenden mit chronologisch zur Flächengröße bezifferten Kürzeln der Tab. 1 entsprechend. 
Tabelle 1: Verwendete Kürzel für die unterschiedenen Flächengrößen und die Anzahl der Replikate jeder Flächengröße.

\begin{tabular}{rrr}
\hline Fläche $\left[\mathbf{m}^{\mathbf{2}}\right]$ & Kürzel & $\mathbf{n}$ \\
\hline 0,0001 & A1 & 200 \\
0,0009 & A2 & 200 \\
0,0100 & A3 & 200 \\
0,0900 & A4 & 200 \\
1,0000 & A5 & 200 \\
9,0000 & A6 & 200 \\
100,0000 & A7 & 50 \\
\hline
\end{tabular}

Bei der Flächenform der Aufnahmeflächen habe ich mich für Quadrate entschieden, welche nach Kreisen den geringsten Randeffekt aufweisen, aber einfacher abzustecken sind (DeNGLER 2003). Flächenformen, die im Verhältnis zu ihrer Größe einen großen Umfang haben, können aus zwei Gründen relativ artenreicher sein, als Flächenformen mit kleinerem Umfang. Je größer die Diagonale einer rechteckigen bzw. der maximale Durchmesser einer runden Aufnahmefläche ist, desto größer ist die Distanz, über die Vegetation erfasst wird. Außerdem erhöht sich der Anteil in die Aufnahmefläche hineinragender Pflanzenteile, was ebenfalls den Artenreichtum beeinflusst.

Die Anordnung der Aufnahmflächen ist ähnlich dem des Carolina Vegetation Survey (CVS), wie es jüngst in einigen amerikanischen Studien Anwendung fand (z. B. PeEt et al. 1998, FridLEy et al. 2005, Goslee 2006). Allerdings habe ich die Anzahl Replikate wie oben ausgeführt erweitert, so dass in jeder Ecke der Aufnahmefläche A7 Serien der Aufnahmeflächen A1 - A6 liegen. Da die Auswahl der Aufnahmeflächen ohne Berücksichtigung von Homogenität oder Vegetationstypen stattfand, war eine Anpassung der Flächengröße der Plots an den jeweiligen Vegetationstyp überflüssig.

Die Serien der Aufnahmeflächen A1-A6 lagen immer dergestalt in den Ecken der Aufnahmefläche A7, dass die äußeren Kanten der Aufnahmefläche A6 den entsprechenden Kanten der Aufnahmefläche A7 entsprachen (Abb. 7). Dadurch waren sie bei jedem Plot gleich positioniert und möglichst weit voneinander entfernt um Autokorrelationseffekte möglichst gering zu halten. Alle anderen Aufnahmeflächen habe ich zufällig platziert, aber immer so, dass die jeweils größere Fläche die kleineren einschließt.

Durch die Einnordung der Plots konnte eine einheitliche Ausrichtung der Plots ermöglicht gewährleistet werden. 
Abbildung 7: Schematische Darstellung

des Aufnahmedesigns (nicht

maßstabsgetreu).

\subsubsection{Vegetationsaufnahmen}

Die Vegetationsaufnahmen sollten ein allen Teilfragen gerecht werdendes Datenset liefern. Um später noch Auswertungen machen zu können, die über eine Bearbeitung der im Rahmen dieser Diplomarbeit aufgeworfenen Fragen hinausgeht, habe ich möglichst viele Aspekte berücksichtigt.

Ich habe die Aufnahmefläche A7 mit einem 50 m Bandmaß abgesteckt, die Aufnahmeflächen A3 - A6 mit mehreren Zollstöcken und die Flächen A1 und A2 mit Plexiglasscheiben, in denen die entsprechende Fläche ausgestanzt war. Berücksichtigt wurden alle in die Flächen hereinragenden Pflanzenteile. Besonders bei den kleineren Flächen musste hierbei dem Umstand Rechnung getragen werden, dass es nicht immer ganz offensichtlich ist, ob z. B. die Blätter eines Baumes, und damit die Art, in die Fläche ragen oder nicht. In solchen Fällen habe ich das Vorhandensein einer Art in der Fläche gemäß der lediglich visuell bestimmbaren Wahrscheinlichkeit vermerkt. War die Art z. B. nur mit einer Wahrscheinlichkeit von $50 \%$ in der Fläche, habe ich statt einer „1“ für anwesend eine „0,5“ notiert. Zwar ist diese visuelle Wahrscheinlichkeitsbestimmung fehlerträchtig und subjektiv, was dazu führt, dass verschiedene Bearbeiter von Fall zu Fall unterschiedlich entscheiden würden. Und doch stellt sie im Rahmen dieser Arbeit und in Bezug auf die Praktikabilität die beste Möglichkeit dar diese Pflanzenteile zu berücksichtigen.

Moose und Flechten können einen erheblichen Anteil der Artenvielfalt ausmachen und sind deshalb in vegetationskundlichen Studien möglichst zu berücksichtigen Dengler (2003: 125). Ich habe bei den Aufnahmen alle makroskopisch erkennbaren Flechten und Moose erfasst.

Die Aufnahmen erstreckten sich über den Zeitraum von Mitte Juni bis Mitte September 2006. Aufgrund dieses engen zeitlichen Rahmens konnte ich die Plots nicht, wie zum Beispiel von DierschKe (1994: 149) zumindest für einige Vegetationstypen gefordert, zweimal innerhalb einer Vegetationsperiode aufsuchen. Dadurch sind jahreszeitlich bedingte Schwankungen der sichtbaren 
Artenzusammensetzung anzunehmen. Besonders frühblühende Geophyten sind dadurch während der kompletten Aufnahmephase nicht mehr bestimmbar gewesen. Im Spätsommer kamen dann die zunehmend verwelkten sommerannuellen Arten hinzu.

Des Weiteren habe ich die Vegetation in verschiedene Schichten gegliedert. Dazu gehörten vier vertikale Schichten, die sich auf die Wuchshöhe sowie das Kriterium verholzend / nicht-verholzend beziehen (Braun-Blanquet 1928: 37). Dengler (2003: 136) folgend nenne ich sie Vertikal-Straten. Es sind dies vom Boden aufwärts:

- Moosschicht (M)

- Krautschicht $(\mathrm{K})$

- Strauchschicht (S)

- Baumschicht (B)

Zur Moosschicht gehören alle auf dem Erdboden wachsenden Flechten und Moose. In die Krautschicht gehörten neben allen krautigen Pflanzen auch Individuen verholzender Pflanzen, deren Größe unter 0,5 m liegt. Die Strauchschicht beinhaltete Bäume und Sträucher mit einer Größe von $0,5 \mathrm{~m}$ bis $5 \mathrm{~m}$. Alle Pflanzen, die größer als $5 \mathrm{~m}$ waren, habe ich schließlich der Baumschicht zugeordnet.

Bei der Aufnahme der Moose und Flechten habe ich außerdem das Substrat vermerkt, wenn diese nicht auf dem Erdboden wuchsen. Daher habe ich die Schichten um folgende Substrat-Straten (DENGLER 2003: 137) ergänzt:

- Epiphytenschicht (E)

- Epixylenschicht (X)

- Epilithenschicht (L)

Durch eine entsprechende Erweiterung der Vertikal-Straten war es möglich eine Unterscheidung zwischen gepflanzten und wild wachsenden Arten vorzunehmen. Einen entsprechenden Hinweis gibt das Kürzel der Schicht, welches dann jeweils um ein " $G$ “ erweitert wurde (KG, SG und BG), wenn die Sippe an dem entsprechenden Standort gepflanzt wurde.

Auf allen Flächengrößen habe ich das vollständige Arteninventar der Gefäßpflanzen, Flechten und Moose entsprechend der oben beschriebenen Schichten erfasst.

Im Gelände habe ich außerdem die folgenden Parameter aufgenommen, wobei ich je nach der räumlichen Variabilität eines Parameters diesen nur für die Aufnahmefläche A7 oder nur für die Aufnahmefläche A6 erhoben habe. Das entsprechende Flächenkürzel steht in Klammern hinter jedem Parameter.

Geografische Lage (A7): Allgemeine Lagebeschreibung zur möglichst einfachen Identifikation des Plots auf einer Karte.

Koordinaten (A6): Gauß-Krüger Koordinaten mit GPS-Empfänger eingemessen.

Landschaftstyp (A7): Bei der Einteilung der Landschaft habe ich mich nach der Topographischen Karte 1:25000, Blatt 2728 Lüneburg gerichtet. Folgende Typen habe ich dabei berücksichtigt:

- Wald (W)

- Grünland (G)

- Ackerland (A) 
- Siedlungsbereich, inklusive Industrie- und Gewerbeflächen (S)

War ein Plot in einer Fläche, deren Definition auf der Karte keiner der oben genannten entsprach, habe ich als Landschaftstyp denjenigen angegeben, der die größte gemeinsame Grenze mit der Ausgangsfläche aufwies.

Nutzungsform (A6): Bei der Definition habe ich nicht nur die Beeinflussung der Aufnahmefläche durch den Menschen berücksichtigt. Es war eine möglichst feine Einteilung erstrebenswert, bei der auch Flächen ohne Nutzung fein differenziert werden. Bei den nachfolgenden Definitionen habe ich mich an Drachenfels (2004) orientiert.

- Scher- und Trittrasen (GR): Überwiegend mehrmals im Jahr gemähte Vegetationsbestände aus Gräsern oder Gräsern und Kräutern in Grünanlagen, Gärten und an Verkehrsflächen.

- Mesophiles Grünland (GM): Mehr oder weniger artenreiches, vergleichsweise extensiv durch Mahd oder Beweidung genutztes Grünland.

- Ruderalflur (UR): Spontan entstandene, nicht landwirtschaftlich genutzte Vegetationsbestände aus Stauden, Gräsern, ein- und zweijährigen Kräutern auf anthropogen stark veränderten, nährstoffreichen Standorten.

- Hausgärten (PH): Wohngebäuden zugeordnete, privat genutzte Zier- und Nutzgärten; i. d. R. jeweils nur zu einem Haus gehörig und durch Hecken oder Zäune von anderen Gärten getrennt.

- Mesophiles Gebüsch (BM): Strauchbestände auf mäßig trockenen bis mäßig feuchten, basenreichen Standorten, meist mit Dominanz von Prunus spinosa (Schlehe), Corylus avellana (Gewöhnliche Hasel), Crataegus spec. (Weißdorn) oder Rosa canina (HundsRose), ausgenommen Hecken.

- Zier- und Ruderalgebüsch / Hecke (BZ): Angepflanzte Gehölzbestände aus Sträuchern und z. T. auch jungen Bäumen im Siedlungsbereich; meist für Zierzwecke, als Sichtoder Lärmschutz, mit mehr oder weniger hohem Anteil an gebietsfremden Arten.

- Bodensaures Laubgebüsch (BS): Strauchbestände auf trockenen bis mäßig feuchten, bodensauren Standorten, ausgenommen Hecken.

- Sand-Magerrasen (RS): Niedrigwüchsige, oft lückige Gras- und Krautfluren auf basenarmen bis basenreichen Sand- und Kiesböden.

- Artenarmes Magerrasen-Stadium (RA): Artenarme Gras- und Krautfluren auf mageren, trockenen bis mäßig feuchten Standorten. Überwiegend infolge von Nutzungsaufgabe bzw. mangelnder Pflege stark an Arten verarmte Sukzessionsstadien von Heiden und Magerrasen, in denen meist Gräser mit breiterer Standortamplitude vorherrschen.

- Befestigte Flächen (TF): Wege, Straßen, Bahnanlagen und Plätze mit befestigter Oberfläche, vegetationslos oder spärlich bewachsen.

- Nadelwald armer Sandböden (WK): Naturnahe und halbnatürliche Nadelwälder auf sehr nährstoffarmen und trockenen oder feuchten Sanden.

- Birken- und Kiefernwald (WV): Entwässerte Birken- und Kiefernbruchwälder; Krautschicht ohne oder mit sehr geringem Anteil von Feuchtezeigern, meist von Dryopteris carthusiana, Rubus spec., Pteridium aquilinum (Adlerfarn) und / oder Deschampsia flexuosa (Draht-Schmiele) dominiert.

- Mesophiler Buchenwald (WM): Buchenwälder auf mäßig trockenen bis mäßig feuchten, mehr oder weniger basenreichen Lehm- und Lössstandorten; Dominanz von 
Fagus sylvatica (meist deutlich mehr als $50 \%$ der ersten Baumschicht), Krautschicht überwiegend aus mesophilen Arten.

- Mesophiler Eichen- Hainbuchen-Mischwald (WC): Mischwälder aus Quercus robur und / oder Carpinus betulus mit Fagus sylvatica u. a. auf mäßig bis gut basenversorgten, mäßig trockenen bis feuchten Standorten außerhalb der Flussauen. Anteil von Fagus sylvatica bis maximal $50 \%$ in der ersten Baumschicht.

- Bodensaurer Buchenwald (WL): Buchenwälder auf mehr oder weniger basenarmen Sand-, Lehm- und Gesteinsböden; Dominanz von Fagus sylvatica (über $50 \%$ in der ersten Baumschicht); in der artenarmen, oft nur spärlich ausgeprägten Krautschicht überwiegend Säurezeiger.

- Bodensaurer Eichenmischwald (WQ): Von Quercus robur oder petraea dominierte Wälder sowie Mischwälder aus Quercus spec. Betula spec. und Pinus sylvestris auf basenarmen Böden; Buchenanteil in der ersten Baumschicht maximal ca. 50 \%; in der Krautschicht überwiegend Säurezeiger.

- Nadelforst (WZ): Gepflanzte bzw. durch forstliche Bewirtschaftung bedingte, meist strukturarme Nadelholzbestände (über $50 \%$ Nadelholzanteil), die deutlich von der potenziell natürlichen Vegetation abweichen.

- Einzelbaum / Baumbestand (HB): Einzelne, landschaftsprägende, vorwiegend alte Bäume sowie Baumgruppen, Baumreihen, Alleen und locker auf größere Flächen verteilte Baumbestände.

- Bestellter Acker (AG): Anbauflächen von Feldfrüchten wie Getreide, Ölpflanzen, Hackfrüchten usw. einschließlich Zwischeneinsaaten.

- Ackerbrache (AB): Unbestellter Acker ohne Spuren vergangener oder zukünftiger Bestellung.

- Landröhricht (NR): Flächenhafte Dominanzbestände von Röhrichtpflanzen auf feuchten bis nassen, allenfalls vorübergehend überfluteten Standorten; zumindest zeitweise mehr oder weniger hochwüchsiger Röhrichtstruktur.

Flächengröße: $\left[\mathrm{m}^{2}\right]$

Hangneigung (A6): Die Hangneigung habe ich in Prozent angegeben. Dabei entsprechen

$$
0^{\circ}=0 \% \text { und } 90^{\circ}=100 \% .
$$

Exposition (A6): Folgende Himmelsrichtungen habe ich unterschieden:

- $\operatorname{Norden}(\mathrm{N})$

- Nordosten (NO)

- Osten $(\mathrm{O})$

- Südosten (SO)

- Süden (S)

- Südwesten (SW)

- Westen (W)

- Nordwesten (NW)

Höhe über NN (A6, [m]): Die Höhe über NN habe ich mit Hilfe der topografischen Karte 1:25000 bestimmt.

Streuanteil (A6, [\%]): Anteil Blätter, Nadeln, Früchte und sonstiger nicht holziger Pflanzenteile. 
Totholzanteil (A6, [\%]): Anteil toter verholzender Pflanzenteile (Äste, Baumstümpfe usw.)

Gesteinsanteil (A6, [\%]): Anteil jeglicher Form von Gestein (Kies, Stein, Mauer, Straße usw.)

Anteil offener Erde (A6, [\%]): Anteil freier Erdflächen, ohne Streu, Totholz Stein oder Vegetation.

Potenzielle Sonnenscheindauer (A6, [h]): Entspricht der maximal möglichen Sonnenscheindauer eines Standortes über die Vegetationsperiode von März bis September. Die ermittelten Werte werden für den gesamten Zeitraum gemittelt und gelten nur für die Krautschicht. Die Spanne reicht von durchschnittlich 0 Stunden Sonnenscheindauer in einem Buchenwald mit dichter Baumkrone bis 13,6 Stunden auf Agrarflächen.

Zur Messung der potenziellen Sonnenscheindauer kam das Horizontoskop nach TonNE (1954) zum Einsatz. Das Gerät besteht aus einer runden Plastikscheibe auf die eine Diagrammscheibe aus Papier gelegt wird (Abb. 8). Die Diagrammscheibe gibt Auskunft über die Sonnenscheindauer zu einer bestimmten Uhrzeit in einem bestimmten Monat, wobei der Umstand berücksichtigt werden muss, dass sich aufgrund der kugelförmigen Gestalt der Erde die potenzielle Sonnenscheindauer bei unterschiedlichen Breitengraden ändert.

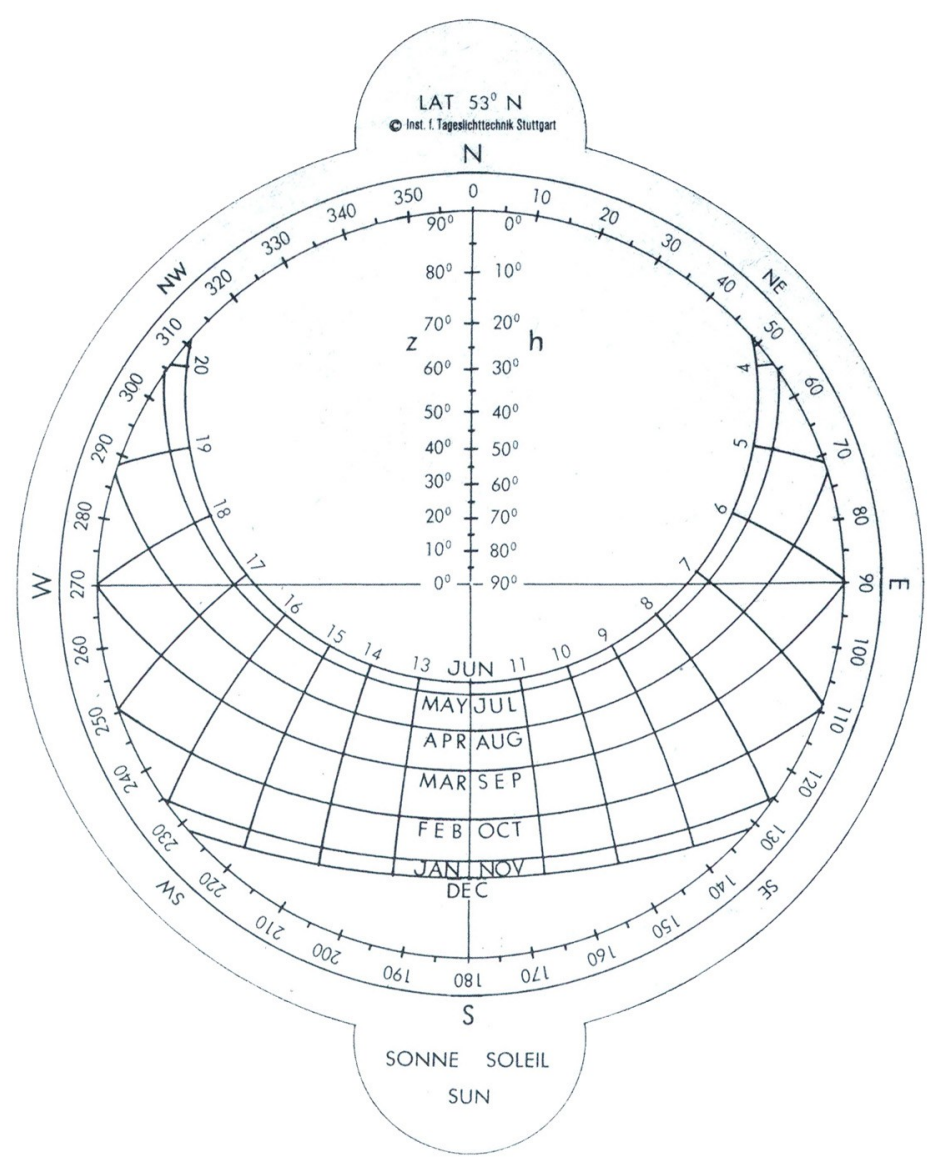

Abbildung 8: Diagrammscheibe für $53^{\circ}$ nördlicher Breite.

Im Feld muss das Gerät mit Hilfe des integrierten Kompasses eingenordet werden. Zur Bestimmung der potenziellen Sonnenscheindauer ist auf den besagten Scheiben eine Plexiglashalbkugel befestigt. In dieser spiegelt sich die Umgebung und ermöglicht dadurch eine Projektion der beschatteten und sonnigen Bereiche auf die Diagrammscheibe. Auf 
dieser können dann die potenziell sonnigen Stunden eines Tages an diesem Standort für die verschiedenen Monate abgelesen werden. Ich habe die potenzielle Sonnenscheindauer in der Mitte der Fläche A6 auf Taillenhöhe (ca. $1 \mathrm{~m}$ ) für die Monate März bis September gemessen. Es galt den Schatten umstehender Bäume oder Gebäude innerhalb der Vegetationsperiode zu berücksichtigen. Dadurch ist die potenzielle Sonnenscheindauer als zusätzlicher abiotischer Faktor hauptsächlich ein Parameter für die Kraut- und Moosschicht. Für die Auswertungen habe ich die durchschnittliche potenzielle Sonnenscheindauer berechnet. Da die Beschattung der Oberfläche durch Wolken mit dem Horizontoskop nicht erfasst werden kann, besitzt dieser Wert nur eine eingeschränkte Aussagekraft. Er stellt aber trotzdem einen guten Wert für den Vergleich verschiedener Aufnahmen miteinander dar, weil angenommen werden kann, dass die durchschnittliche Bewölkung an allen Standorten des Untersuchungsgebietes gleich ist.

\subsection{Begriffsdefinitionen}

Im Rahmen dieser Arbeit verwende ich die folgenden Begriffe im Sinne der hier festgelegten Definitionen.

- Art: Unter einer Art verstehe ich alle unterschiedenen Sippen oder Taxa unabhängig von ihrem Klassifikationsniveau, wobei die Angaben unter Kap. $3.5 \mathrm{zu}$ beachten sind. Eine Art stellt die gängigste Einheit von Biodiversitäts-Untersuchungen dar. Dabei gibt es für die Fülle von Artengruppen, die zusammen die Biodiversität ausmachen, auch verschiedene Ansätze taxonomischer Klassifikation. Selbst unter den behandelten Artengruppen existieren unterschiedliche Klassifikationsansätze. Ob eine Sippe als eigenständige Art, Unterart oder als Varietät eingestuft wird, wird von spezialisierten Taxonomen fortwährend diskutiert. Daraus resultierend ist die Nomenklatur ständigen Änderungen unterworfen.

- Artenzahl: Flächenunabhängige Zahl von Arten. Ohne Bezugsrahmen wenig aussagekräftig. Artenzahlen bekommen vor allem dann eine Bedeutung, wenn man sie in Bezug zu einer bestimmten Flächengröße setzt (s. Artendichte).

- Artendichte: Unter Artendichte wird eine bestimmte Anzahl von Arten auf einer bestimmten Flächengröße verstanden. Sie ist wesentlicher Bestandteil der folgenden Auswertungen, da sich nahezu alle Angaben von Artenzahlen auf die angegebenen Flächengrößen beziehen.

- Artenvielfalt: Die Artenvielfalt ist ein Oberbegriff für alles, was die Verteilung von Arten im Raum betrifft. Dadurch stellt die Artendichte einen Teilaspekt der Artenvielfalt dar.

\subsection{Datenauswertung}

Für die Auswertung der Daten und die Bearbeitung der Fragen im folgenden Kapitel habe ich neben den bereits erwähnten erhobenen Standortparametern noch den floristischen Status und den RoteListe-Status jeder Pflanzenart in meine Datenbank aufgenommen. Bei den Gefäßpflanzenarten, die 
an ihrem Fundort angepflanzt worden waren, fand der Rote-Liste-Status keine Berücksichtigung.

Die Zuordnung der Arten zu einem bestimmten floristischen Status richtet sich bei den Gefäßpflanzen nach Wisskirchen \& Haeupler (1998). Bei den Moosen und Flechten (im Untersuchungsgebiet im Wesentlichen indigene Arten) wurden die Angaben ergänzt.

Ich habe beim floristischen Status die folgenden vier Gruppen unterschieden:

- Indigene Arten: Pflanzensippen, die im Untersuchungsgebiet einheimisch sind, sich dort ohne Einfluss des Menschen ausgebreitet haben und dort dauerhaft vorkommen und sich reproduzieren.

- Neophyten: Pflanzensippen, die vor $1492^{2}$ nicht im Untersuchungsgebiet wuchsen, unabhängig vom Grad ihrer Etablierung.

- Archaeophyten: Pflanzenarten, die vor 1492 im Untersuchungsgebiet wuchsen, dort aber natürlicherweise nicht heimisch sind.

- Kultivierte Arten: Kultivierte Arten umfassen Nutzpflanzen innerhalb und außerhalb Agrarflächen, die im Untersuchungsgebiet nicht zur potenziell natürlichen Vegetation gehören.

Unter den floristischen Statusgruppen sind es vor allem die Neophyten, deren Verbreitung, Dichte und Häufigkeit in den letzten Jahren viel erforscht worden sind (KowARIK 2003). Dabei handelt es sich meist um naturschutzfachliche Aspekte. Archaeophyten haben sich seit längerer Zeit etabliert und entziehen sich weitestgehend der Möglichkeit und der Notwendigkeit ihren Einfluss auf die heimische Flora zu hinterfragen. Da es zu den Neophyten eine große Menge an Daten und Literatur gibt, finden bei den Auswertungen der Daten zu den floristischen Statusgruppen die Neophyten am meisten Beachtung.

Beim Rote-Liste-Status habe kamen Rote Listen unterschiedlicher Bezugsregionen zum Einsatz. Anwendung fanden die Rote Liste für Gesamtdeutschland vom Bundesamt Für NAturschutz (1996), sowie die Rote Liste für Niedersachsen gesamt und das niedersächsische Binnentiefland. Hier kamen für die drei Artengruppen unterschiedliche Rote Listen zum Einsatz:

- Gefäßpflanzen: GARve (2004)

- Moose: Koperski (1999)

- Flechten: Hauck (1992)

Alle Rote Listen folgen der Einstufung des Bundesamtes für Naturschutz (1996):

- 0: ausgestorben oder verschollen

- 1: vom Aussterben bedroht

- 2: stark gefährdet

- 3: gefährdet

- R: sehr selten, aber aktuell nicht gefährdet

- G: Gefährdung anzunehmen, aber unklar, wie stark

- D: Datenlage unsicher, d. h. unklar, ob gefährdet oder nicht

- V: zurückgehend, aber aktuell nicht gefährdet (Vorwarnliste) [nur bei Moosen]

- -: fehlend

2 Man geht davon aus, dass sich seit der Entdeckung Amerikas und dem damit verbundenen interkontinentalen Schiffverkehrs die Ausbreitung gebietsfremder Arten stark erhöht hat (vgl. KowARIK 2003). 
- ?: Vorkommen fraglich

- *: ungefährdet

Für die Digitalisierung und Sortierung der Vegetationsdaten wurde das Programm SORT 4.0 von AcKermann \& DuRKa (1998) verwendet. Einfache Berechnungen von Artenzahlen und Biodiversitäts-Indizes wurden ebenfalls mit diesem Programm durchgeführt. Werte deskriptiver Statistik sowie einfache Balkendiagramme wurden mit Microsoft Excel 2002 oder OpenOffice.org Calc ermittelt bzw. erstellt. Histogramme, Box-Whisker-Plots, nicht-lineare Regressionen bei der Approximation der Artenzahl-Arealkurven (s. 3.4.3), ANOVAs (s. S. 26) sowie Tests auf Normalverteilung habe ich mit Statistica 7.1 erstellt bzw. durchgeführt.

\subsection{Statistische Verfahren}

\subsubsection{Artenzahlen auf unterschiedlichen Flächengrößen}

Im folgenden werden die statistischen Größen definiert, mit denen ich bei den Auswertungen operiert habe (Tab. 2). Die Definitionen folgen, wenn nicht anders angegeben, LozÁN \& KaUsCH (2004).

Tabelle 2: Definitionen verwendeter Größen deskriptiver Statisitik sowie des Bestimmtheitsmaßes.

\begin{tabular}{lcl}
\hline Name & Kürzel & Funktion \\
\hline Arithmetisches Mittel & $\varnothing$ & $\varnothing=\frac{\sum x_{i}}{n}$ \\
Standardabweichung & SD & $S D=\sqrt{\frac{\sum\left(x_{i}-\bar{x}\right)^{2}}{n-1}}$ \\
Schiefe & $\mathrm{F}$ & $F=\frac{m_{3}}{s^{3}}$ \\
Kurtosis & $\mathrm{K}$ & $K=\frac{m_{4}}{s^{4}}$ \\
Variationskoeffizient & $\mathrm{V}$ & $V=\frac{S D}{\varnothing}$ \\
Bestimmtheitsma & $\mathrm{R}^{2}$ & $R^{2}=\frac{\left(\sum x y\right)^{2}}{\sum x x \cdot \sum y y}$ \\
\hline
\end{tabular}


Abgesehen von der Präsentation der durchschnittlichen Artenzahlen (Kap. 4.1.1) habe ich für die Auswertungen die Artendichten der Flächen A1 - A6 innerhalb der 50 Plots gemittelt. Dadurch ergaben sich für jede Flächengröße 50 Werte und für jede Flächengröße galt, dass diese 50 Werte innerhalb des Untersuchungsgebietes zufallsverteilt waren.

Für die logarithmische Transformation muss ich bei Anwesenheit von Nullwerten diesen einen Wert zuweisen, der sich logarithmieren lässt, da

$$
\log 10(0)=-\infty
$$

ist. Unter der Annahme, dass 1 der minimal messbare Wert größer als 0 ist, schlagen LozÁN \& Kausch (2004) vor, zu jedem Wert z. B. 0,5 zu addieren. Da ich bei der Mittelung der Artendichten als minimalen Wert 0,25 erhalten kann, habe ich zu jedem Nullwert die Hälfte davon, also 0,125 addiert.

Die Überprüfung, ob die Artendichten normalverteilt sind (s. 4.2), habe ich nach einem Kolmogoroff-Smirnoff-Test durchgeführt. Unter der Annahme der Nullhypothese, dass die Artendichten nicht normalverteilt seien, bedeutet ein Signifikanzniveau $p>0,20$ das eine Abweichung von der Normalverteilung nicht anzunehmen ist.

Bei Korrelationen habe ich den Korrelationskoeffizienten nach Pearson (r) berechnet, wobei ich das Signifikanzniveau auf $\mathrm{p}<0,05$ festgelegt habe.

Die Kurvenanpassungen der Standardabweichung und des Variationskoeffizienten in Abhängigkeit zur logarithmierten Flächengröße (s. 4.4) habe ich mit nicht-linearen Regressionen durchgeführt. Dabei habe ich die Signifikanzniveaus $\mathrm{p}<0,001$ (hochsignifikant) und $\mathrm{p}<0,05$ (signifikant) unterschieden.

Zur Untersuchung der Standardabweichung der Artendichte in Abhängigkeit zur Flächengröße kam eine Exponentialfunktion folgender Form zum Einsatz:

$$
S D=c \cdot e^{z \cdot \log 10(A)}
$$

mit $\mathrm{A}=$ Fläche sowie $\mathrm{c}$ und $\mathrm{z}$ als Funktionsparameter. Eine Approximation des Variationskoeffizienten in Abhängigkeit zur Flächengröße erfolgte mit einer polynomischen Funktion zweiten Grades der Form

$$
V=c \cdot \log 10(A)^{2}+z \cdot \log 10(A)+b
$$

mit $\mathrm{c}, \mathrm{z}$ und $\mathrm{b}$ als Funktionsparameter. 


\subsubsection{Arten-Areal-Beziehungen}

Um herauszufinden, welche Funktion die Arten-Areal-Beziehung am besten beschreibt, habe ich mehrere Funktionen getestet (Tab. 3).

Tabelle 3: Name, Funktion und Quelle der im Rahmen der Untersuchung zur Arten-Areal-Beziehung getesteten Kurven.

\begin{tabular}{lll}
\hline Name & Funktion & Quelle \\
\hline Lineare Funktion & $S=c \cdot A+z$ & Connor \& McCoy (1979) \\
Potenzfunktion & $S=c \cdot A^{z}$ & Arrhenius (1920, 1921) \\
Logarithmusfunktion & $S=c \cdot \log 10(A)+z$ & Gleason (1922, 1925) \\
Michaelis-Menten-Funktion & $S=\frac{c \cdot A}{A+z}$ & Schmitt (1999) \\
$\begin{array}{l}\text { Michaelis-Menten-Funktion, transformiert } \\
\text { nach Hanes (1932) }\end{array}$ & $\frac{A}{S}=\frac{A}{c}+z$ & Schmitt (1999) \\
\hline
\end{tabular}

Die Kurven der linearen Funktion, der Potenzfunktion sowie der Logarithmusfunktion habe ich zudem im logarithmierten Raum approximiert. Dadurch waren insgesamt sieben Funktionen zu testen. Die Michaelis-Menten-Funktion habe ich außerdem in einer transformierten Form nach Hanes (1932, zitiert nach Schmitт 1999) getestet:

Diese Transformation bildet durch Kehrwertbildung eine linearisierte Form der originalen Michaelis-Menten-Funktion und ermöglicht dadurch eine lineare Regression. Die lineare Funktion und die Logarithmusfunktion im logarithmierten Raum habe ich ebenfalls mit linearen Regressionen approximiert, wohingegen die logarithmierten Funktionen sowie beide Varianten der Potenzfunktion mit nicht-linearen Regressionen angepasst wurden.

Um herauszufinden, welche Beziehung den Zusammenhang von Artenzahl und Arealgröße am besten beschreibt, habe ich vier Gütekriterien aufgestellt anhand derer die Qualität einer Funktion bemessen werden sollte:

- Das Bestimmtheitsmaß $\left(\mathrm{R}^{2}\right)$ : Kann definiert werden als das Verhältnis des Anteils der durch die Regression erklärten Streuung zur Gesamtstreuung. Je höher das Bestimmtheitsmaß ist, desto mehr kann die Streuung der Artenzahl durch die Abhängigkeit zur Flächengröße erklärt werden.

- Mittlerer relativer Fehler: Er stellt die über alle Flächengrößen gemittelte Relation der Residuen zur empirich ermittelten Artenzahl dar. Ein Residuum ist der sogenannte Restwert bei einer Regression. Er stellt den Anteil an Varianz dar, der im Rahmen der Regression nicht erklärt werden kann. Mit Hilfe der Funktionsparameter einer bestimmten Funktion kann man für jede Flächengröße die Differenz eines Schätzwertes (mit der Funktion berechnete Artenzahl) und der empirisch ermittelten Artenzahl berechnen und erhält damit das Residuum.

- Maximaler relativer Fehler: Der maximale relative Fehler ist definiert als die größte relative Abweichung eines Schätzwertes zur ermpirisch ermittelten Artenzahl unter allen 
berücksichtigten Flächengrößen.

- Relativer Extrapolationsfehler: Dieser Fehler stellt den prozentualen Fehler einer berechneten Artenzahl (also eines Schätzwertes) für die Fläche A7 zur empirisch ermittelten Artenzahl auf dieser Fläche dar. Allerdings wurden die der Berechnung zugrunde liegenden Funktionsparameter zuvor in einer Kurvenanpassung ermittelt, die ohne Berücksichtigung der Artenzahl der Fläche A7 durchgeführt wurde. Bei dieser Berechnung unterstelle ich also, im Rahmen der Vegetationsaufnahmen die Artendichte auf dieser Fläche nicht erfasst zu haben, so dass zur Ermittlung der Artendichte eine Berechnung mit Hilfe der Funktion erfolgen müsste.

Die Funktionen habe ich anhand dieser Gütekriterien für jeden Plot einzeln getestet. Anschließend habe ich ein Ranking durchgeführt, bei dem jeder der sieben Funktionen für jeden Plot einen Rang bekommen hat. Anschließend habe ich die Summe der Ränge durch die Anzahl der Plots (sprich 50) geteilt und habe damit das mittlere Ranking einer Funktion für ein bestimmtes Gütekriterium erhalten. Diese Prozedur habe ich mit allen vier Gütekriterien durchgeführt. Dadurch hatte ich für jede Funktion vier mittlere Rankings. Abschließend habe ich diese mittleren Rankings der Gütekriterien für jede Funktion gemittelt. Dieser Wert stellt den abschließenden Rang einer Funktion bezogen auf deren Güte bei der Approximation der Artendichte dar.

In der Literatur wird die Steigung der Artenzahl-Areal-Verläufe meist als z-Wert bezeichnet. Dabei ist zu unterscheiden zwischen dem z-Wert einer Funktion und dem z-Wert im Sinne der Steigung einer Artenzahl-Areal-Kurve. Der z-Wert einer Potenzfunktion gibt zwar auch deren Steigung wieder, der aber nicht zur Beurteilung der Steigung geeignet ist und dies umso weniger, wenn man sich für die Steigung einer Artenzahl-Areal-Kurve an einem bestimmten Flächenübergang interessiert, da er der Steigung des größten Flächenüberganges entspricht. Deshalb habe ich die Potenzfunktion logarithmiert, wodurch sie eine lineare Form erhält. Die Steigung einer linearen Funktion lässt sich berechnen als die Differenz der Artendichten von der einen zur nächstgrößeren Fläche geteilt durch die Differenz der entsprechenden zwei Flächengrößen. Dies geschah entsprechend der logarithmierten Form der Potenzfunktion, also im doppelt-logarithmischen Raum mit logarithmierten Artenzahlen und logarithmierter Flächengröße. Bei den floristischen Statusgruppen habe ich mich direkt der logarithmierten mittleren Artenzahlen der 50 Plots bedient, um die z-Werte zu berechnen.

Um zu prüfen, ob sich die Mittelwerte der z-Werte verschiedener Flächengrößen signifikant voneinander unterscheiden, kamen ANOVAs (Analysis of variance) zum Einsatz. Im Rahmen eines Post-hoc-Verfahrens, habe ich sie mit einem Tukey HSD-Test auf homogene Gruppen überprüft (Quinn \& KeOugh 2002).

Die Mittelung der z-Werte erfolgte zunächst für alle Flächenübergänge über alle Plots, wobei ich bei den Flechten und den Moosen Plots ausgelassen habe, auf denen keine Art vorkam. Für einen endgültigen, vergleichbaren Wert habe ich die z-Werte über alle Flächenübergänge gemittelt.

Gemäß der Bezeichnung der unterschiedlichen Flächengrößen, habe ich die Flächenübergänge für die Berechnung der z-Werte mit Kürzeln von A1-A2 bis A6-A7 versehen. 


\subsection{Nomenklatur und Bestimmungshilfen}

$\mathrm{m}$ Folgenden ist die zur Bestimmung der Sippen herangezogene Literatur angeben. Neben Nomenklatur und Bestimmungsschlüsseln umfasst diese Auflistung auch Bild- oder Zeichenbände, die mir zur visuellen Überprüfung der Bestimmungsergebnisse gedient haben.

\subsubsection{Blütenpflanzen und Farne}

Die Nomenklatur der Gefäßpflanzen richtet sich (für die lateinischen und deutschen Namen) nach JäGER \& WeRNER (2005).

Die Bestimmung der Gefäßpflanzen erfolgte im Wesentlichen nach JäGER \& WerNER (2005) und Oberdorfer (2001). Zur Bestimmung der Poaceae (Süßgräser) habe ich Conert (2000) herangezogen.

Einige Arten der Gattung Carex (Seggen) habe ich nach Jermy, Chater \& David (1982) sowie Eisele \& ZäHringer (1997) bestimmt. Für die vegetative Bestimmung einiger Arten der Familie Apiaceae (Doldenblütler) habe ich Tutin (1980) sowie Adler, Oswald \& Fischer (1994) herangezogen.

Bei der Bestimmung der Keimlinge habe ich Hanf (1990), Muller (1978) sowie Csapody (1968) verwendet.

Zur visuellen Überprüfung der Bestimmungsergebnisse kam außerdem HaEuPLER \& MuER (2000) zum Einsatz.

Da ich für die Bearbeitung der Fragen keine genaue Kenntnis jeder einzelnen verschiedenen Sippe brauche, habe ich einige schwierige Gruppen nur bis zum Gattungsniveau oder auf Aggregatsebene bestimmt.

Folgende Sippen habe ich zu Artengruppen zusammengefasst:

- Achillea millefolium agg. (Schafgarbe)

- Festuca ovina agg.(Schaf-Schwingel): In den meisten Fällen habe ich die Subspezies dieses Aggregats nicht voneinander getrennt.

- Festuca rubra agg. (Rot-Schwingel): In den meisten Fällen habe ich die Subspezies dieses Aggregats nicht voneinander getrennt.

- Hieracium subgen. Pilosella (Mausohr-Habichtskräuter)

- Potentilla argentea (Silber-Fingerkraut)

- Taraxacum (Löwenzahn): Diese sippenreiche Gattung habe ich in Einzelfällen bis zur Sektion bestimmt. Da dies aber nicht kontinuierlich geschah, habe ich bei der Auswertung keine Arten oder Sektionen mehr unterschieden.

- Rubus coryllifolius agg. (Haselblattbrombeeren) und Rubus fruticosus agg. (Brombeeren), die beiden häufigsten Aggregate der Gattung im Untersuchungsgebiet, habe ich nicht voneinander unterschieden. Ein nicht unerheblicher Teil der unter Rubus coryxllifolius agg. aufgenommenen Arten gehört zum Rubus fruticosus agg..

Folgende Sippen hingegen habe ich unterschieden und in der Auswertung als Art aufgefasst:

- Aira caryophyllea ssp. caryophyllea (Nelken-Haferschmiele)

- Calystegia sepium ssp. sepium (Gewöhnliche Zaunwinde) 
- Carduus nutans ssp. nutans (Nickende Distel)

- Daucus carota ssp. carota (Wilde Möhre)

- Galium palustre ssp. palustre (Sumpf-Labkraut)

- Geranium robertianum ssp. robertianum (Stinkender Storchschnabel)

- Heracleum sphondylium ssp. sphondylium (Wiesen-Bärenklau)

- Herniaria glabra ssp. glabra (Kahles Bruchkraut)

- Plantago major ssp. intermedia (Kleiner Wegerich)

- Plantago major ssp. major (Großer Wegerich)

- Portulaca oleracea ssp. oleracea (Gemüse-Portulak)

In dieser Darstellung nicht berücksichtigt sind Subspezies, die die einzige in Deutschland vorkommende Sippe der Art darstellen (z. B. Silene latifolia ssp. alba).

Im folgenden Fall bin ich von der angegebenen Nomenklatur abgewichen:

- Elytrigia repens habe ich WissKIRCHEN \& HAEUPLER (1998) folgend Elymus repens benannt. Von den unterschiedenen Subspezies ist im Untersuchungsgebiet nur Elymus repens ssp. repens bekannt, weshalb die Benennung im Folgenden auch nur auf Artniveau erfolgt.

Die Nomenklatur der Farne folgt JäGER \& Werner (2005). Zur Bestimmung habe ich überwiegend Wellinghausen (1997) zu Rate gezogen. In Zweifelsfällen kam auch hier JäGER \& Werner (2005) zum Einsatz.

\subsubsection{Moose und Flechten}

Bei der Nomenklatur der Moose habe ich mich nach Koperski et al. (2000) gerichtet. Zur Bestimmung habe ich hauptsächlich Frahm \& Frey (2003) sowie SMith (2006) benutzt. Zur visuellen Überprüfung der Bestimmungsergebnisse habe ich LANDweHR (1966) herangezogen. Als Nachschlagewerk sowie durch zahlreiche hilfreiche Fotografien war mir außerdem MALCOLM \& Malcolm (2000) eine Hilfe.

Bei dem Exemplar der Sippe Schistidium apocarpum agg. habe ich die Kleinart nicht bestimmt.

In folgenden Fällen habe ich Subspezies bzw. Varietäten unterschieden, die ich in den Auswertungen als Art aufgefasst habe:

- Ceratodon purpureus ssp. purpureus

- Hypnum cupressiforme var. cupressiforme

- Hypnum cupressiforme var. lacunosum

- Racomitrium canescens ssp. canescens

Die Nomenklatur und die Bestimmung der Flechten folgt WIRTH (1995). In einigen Fällen habe ich mich bei der Bestimmung außerdem Purvis et al. (1992) und Dobson (2000) bedient.

Folgende Subspezies habe ich differenziert und betrachte sie bei den Auswertungen als Art:

- Cladonia furcata ssp. furcata 


\section{Artenzahlen auf unterschiedlichen Flächengrößen}

Nach einer Darstellung der absoluten sowie mittleren Artenzahlen der unterschiedlichen Flächengrößen soll es in diesem Kapitel um die relativen Anteile der unterschiedenen Artengruppen sowie der floristischen Statusgruppen gehen. Des Weiteren wird beispielhaft die Verteilung der Artendichten sowie die Standardabweichung und der Variationskoeffizient in Abhängigkeit zur Flächengröße untersucht.

\subsection{Absolute und mittlere Artenzahlen}

\subsubsection{Ergebnisse}

Auf den 50 Plots kommen insgesamt 585 Pflanzenarten vor, wobei die Angaben, wie unter Kap. 3.5 beschrieben, zu berücksichtigen sind. Den größten Anteil an der Artendichte haben die Gefäßpflanzen mit insgesamt 476 Arten $(81,4 \%)$. Bei den Flechten sind es insgesamt 60 Arten (10,3\%), bei den Moosen 49 (8,3\%). Unter diesen Arten sind 388 indigene Arten (66,3\%), 125 Neophyten (21,4\%), 27 Archaeophyten (4,6 \%), 9 kultivierte Arten (1,5\%), 1 unbeständige Art $(0,2 \%)$ und 35 Arten mit unbestimmtem floristischen Status $(6,0 \%)$. Unter den Flechten und Moosen befinden sich keine Neophyten oder Archaeophyten. Demnach gehören alle Arten einer dieser floristischen Statusgruppen zu den Gefäßpflanzen, unter denen sie einen Anteil von ca. $32 \%$ haben. Von den 125 erfassten Neophyten kommen 96 ausschließlich gepflanzt vor, 21 ausschließlich wild und 8 sowohl angepflanzt als auch wild. Im arithmetischen Mittel haben indigene Arten mit mehr als 85 \% Artenanteil auf allen Flächengrößen die größte Abundanz.

Die arithmetischen Mittel, Standardabweichungen, Minima und Maxima der Artenzahlen auf den verschiedenen Flächengrößen sowie deren Median, sind in Tabelle 4 angegeben. Ihre Ermittlung folgt den unter 3.4.2 festgelegten Definitionen. Eine grafische Darstellung der Gesamtartenzahlen zeigt Abbildung 9.

Da einer der Plots auf einer Agrarfläche lag, die kurz vor der Vegetationsaufnahme umgegraben wurde, sind die minimalen Artenzahlen aller Gruppierungen auf allen Flächengrößen fast immer 0. Die durchschnittlichen Gesamtartenzahlen reichen von 1,2 auf der kleinsten bis 38,7 auf der größten Fläche (Tab. 4). Arithmetisches Mittel und Median stimmen hier stets gut überein, die Standardabweichung ist auf allen Flächengrößen deutlich kleiner als das arithmetische Mittel. Dies trifft auch auf die Gefäßpflanzen zu. Bei den Moosen sind sich arithmetisches Mittel und Median erst ab der Fläche A4 ähnlich, während die Standardabweichung erst ab der Fläche A5 gleich oder kleiner als das arithmetische Mittel ist. Bei den Flechten ist die Standardabweichung auf allen Flächen größer als das arithmetische Mittel, was für eine geringe Aussagekraft des arithmetischen Mittels bei dieser Artengruppe spricht. Lediglich auf der Fläche A7, auf der sich das arithmetische Mittel der Flechten im Vergleich zur Fläche A6 mehr als verdreifacht, ist der Median größer als Null. Ähnliches gilt für die floristischen Gruppen, ausgenommen den indigenen Arten. Die Standardabweichung ist bei Neophyten, Archaeophyten, kultivierten Arten und unbeständigen 
Arten auf allen Flächen größer als das arithmetische Mittel, während der Median gleich Null ist. Einzige Ausnahme stellt der Median der Neophyten dar, obwohl dieser ist aber trotzdem um fast $75 \%$ kleiner als das arithmetische Mittel ist.

Auch bei den floristischen Statusgruppen hat das arithmetische Mittel wenig Aussagekraft. Gepflanzte Arten waren im Durchschnitt häufiger frequentiert als Neophyten. Als Extremwert fällt das Maximum der Gesamtartenzahl auf der Fläche A7 auf, die sich im Vergleich zur Fläche A6 mehr als verdoppelt. Außerdem ist der hohe Anteil gepflanzter Arten zu erwähnen mit einem arithmetischen Mittel von 4,3 Arten und einem Maximum von 84 Arten auf der Fläche A7. Allerdings stellt die Standardabweichung das dreifache des arithmetischen Mittels dar und der Median liegt auch hier bei Null. Die gepflanzten Arten sind also ebenfalls extrem ungleich verteilt.

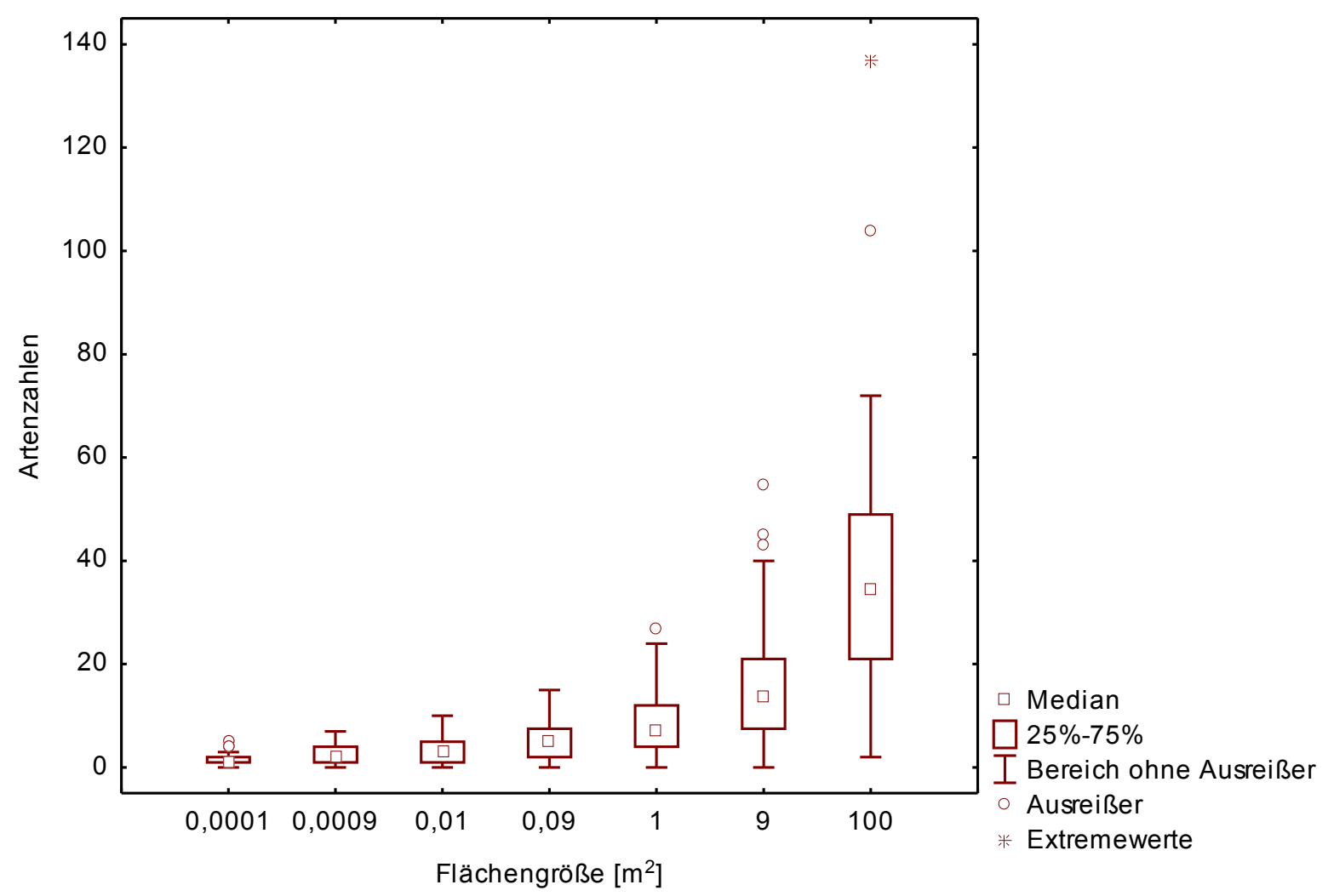

Abbildung 9: Box-Whisker-Plot der absoluten Artenzahlen auf allen Flächengrößen. Ausgenommen der Fläche A7 ( $\mathrm{n}=50)$ gilt: $\mathrm{n}=200$. Die Ausreißer sind größer bzw. kleiner als die Ober- bzw. Unterwerte der Box $+1 *$ Box-Bereich. Die Extremwerte berechnen sich durch die Summe der Ober- bzw. Unterwerte der Box $+2 *$ Box-Bereich. 
Tabelle 4: Arithmetisches Mittel (Ø), Standardabweichung (SD), Median (Med.), Minimum (Min.) und Maximum (Max.) der Artenzahlen insgesamt, differenziert nach Artengruppen, Statusgruppen sowie der gepflanzten Arten.

\begin{tabular}{lrrrrrrrrrrrrrrrrrrrrr}
\hline Artzahlen & $\varnothing$ & $S D$ & Med. & Min. & Max. & $\varnothing$ & SD & Med. & Min. & Max. & $\varnothing$ & SD & Med. & Min. & Max. & $\varnothing$ & SD & Med. & Min. & Max. \\
\hline Gesamt & 1,63 & 1,2 & 1 & 0 & 5 & 2,39 & 1,7 & 2 & 0 & 7 & 3,46 & 2,4 & 3 & 0 & 10 & 5,11 & 3,4 & 5 & 0 & 15 \\
Gefäßpflanzen & 1,35 & 1,0 & 1 & 0 & 5 & 1,90 & 1,4 & 2 & 0 & 7 & 2,71 & 2,0 & 2 & 0 & 9 & 3,92 & 2,8 & 3 & 0 & 13 \\
Moose & 0,27 & 0,6 & 0 & 0 & 3 & 0,43 & 0,8 & 0 & 0 & 5 & 0,67 & 1,0 & 0 & 0 & 5 & 1,03 & 1,3 & 1 & 0 & 6 \\
Flechten & 0,01 & 0,1 & 0 & 0 & 1 & 0,06 & 0,3 & 0 & 0 & 3 & 0,09 & 0,4 & 0 & 0 & 4 & 0,17 & 0,7 & 0 & 0 & 5 \\
Indigene Arten & 1,39 & 1,3 & 1 & 0 & 5 & 2,05 & 1,8 & 2 & 0 & 7 & 2,97 & 2,5 & 3 & 0 & 10 & 4,40 & 3,4 & 4 & 0 & 14 \\
Neophyten & 0,08 & 0,3 & 0 & 0 & 3 & 0,10 & 0,4 & 0 & 0 & 4 & 0,14 & 0,5 & 0 & 0 & 4 & 0,22 & 0,6 & 0 & 0 & 4 \\
Archaeophyten & 0,01 & 0,1 & 0 & 0 & 1 & 0,02 & 0,1 & 0 & 0 & 1 & 0,06 & 0,2 & 0 & 0 & 2 & 0,12 & 0,4 & 0 & 0 & 2 \\
Kultivierte Arten & 0,09 & 0,3 & 0 & 0 & 1 & 0,12 & 0,3 & 0 & 0 & 1 & 0,15 & 0,4 & 0 & 0 & 1 & 0,17 & 0,4 & 0 & 0 & 1 \\
Unbeständige Arten & 0,04 & 0,2 & 0 & 0 & 1 & 0,05 & 0,2 & 0 & 0 & 1 & 0,06 & 0,2 & 0 & 0 & 1 & 0,06 & 0,2 & 0 & 0 & 1 \\
Gepflanzt Arten & 0,22 & 0,5 & 0 & 0 & 3 & 0,27 & 0,5 & 0 & 0 & 4 & 0,34 & 0,7 & 0 & 0 & 5 & 0,41 & 0,8 & 0 & 0 & 5 \\
\hline
\end{tabular}

\begin{tabular}{|c|c|c|c|c|c|c|c|c|c|c|c|c|c|c|c|}
\hline \multirow{2}{*}{$\begin{array}{l}\text { Flächengröße } \\
\text { Artzahlen }\end{array}$} & \multicolumn{5}{|c|}{$1 \mathrm{~m}^{2}$} & \multicolumn{5}{|c|}{$9 \mathrm{~m}^{2}$} & \multicolumn{5}{|c|}{$100 \mathrm{~m}^{2}$} \\
\hline & $\varnothing$ & $S D$ & Med. & Min. & Max. & $\varnothing$ & $S D$ & Med. & Min. & Max. & $\varnothing$ & $S D$ & Med. & Min. & $\overline{\operatorname{Max} .}$ \\
\hline Gesamt & 8,50 & 5,6 & 7 & 0 & 27 & 15,18 & 9,8 & 14 & 0 & 55 & 38,70 & 25,5 & 34,5 & 2 & 137 \\
\hline Gefäßpflanzen & 6,38 & 4,7 & 5 & 0 & 23 & 11,33 & 8,0 & 10 & 0 & 48 & 28,62 & 20,4 & 27,0 & 1 & 117 \\
\hline Moose & 1,56 & 1,6 & 1 & 0 & 7 & 2,56 & 2,2 & 2 & 0 & 12 & 5,78 & 4,6 & 5,5 & 0 & 20 \\
\hline Flechten & 0,47 & 1,2 & 0 & 0 & 7 & 1,29 & 2,2 & 0 & 0 & 12 & 4,30 & 4,4 & 3,0 & 0 & 14 \\
\hline Indigene Arten & 7,17 & 5,4 & 6 & 0 & 26 & 12,63 & 8,3 & 12 & 0 & 43 & 31,28 & 18,2 & 30,0 & 1 & 67 \\
\hline Neophyten & 0,44 & 1,2 & 0 & 0 & 10 & 1,18 & 3,1 & 0 & 0 & 27 & 3,84 & 9,5 & 1,0 & 0 & 60 \\
\hline Archaeophyten & 0,24 & 0,6 & 0 & 0 & 3 & 0,48 & 0,9 & 0 & 0 & 4 & 1,50 & 1,9 & 0,5 & 0 & 6 \\
\hline Kultivierte Arten & 0,20 & 0,4 & 0 & 0 & 2 & 0,23 & 0,5 & 0 & 0 & 2 & 0,34 & 0,6 & 0,0 & 0 & 2 \\
\hline Unbeständige Arten & 0,06 & 0,2 & 0 & 0 & 1 & 0,07 & 0,2 & 0 & 0 & 1 & 0,08 & 0,3 & 0,0 & 0 & 1 \\
\hline Gepflanzte Arten & 0,60 & 1,3 & 0 & 0 & 10 & 1,31 & 3,9 & 0 & 0 & 36 & 4,26 & 13,5 & 0,0 & 0 & 84 \\
\hline
\end{tabular}


Betrachtet man die Artenzahlen der unterschiedlichen Landschaftstypen, befanden sich im Siedlungsbereich die durchschnittlich artenreichsten Plots, auf Agrarflächen (Acker) die durchschnittlich artenärmsten (Abb. 10).

Dabei finden sich im Grünland und im Siedlungsbereich durchschnittlich mehr Flechtenarten als in Wäldern. Die durchschnittlichen Artenzahlen der Flechten und Moose sind im Grünland und im Siedlungsbereich sehr ähnlich. Die Plots mit den durchschnittlich meisten Moosarten befanden sich in den Wäldern. Die Artenzahlen der Waldplots haben insgesamt die geringste Standardabweichung, die der Agrarflächen die relativ zur mittleren Artenzahl höchste.

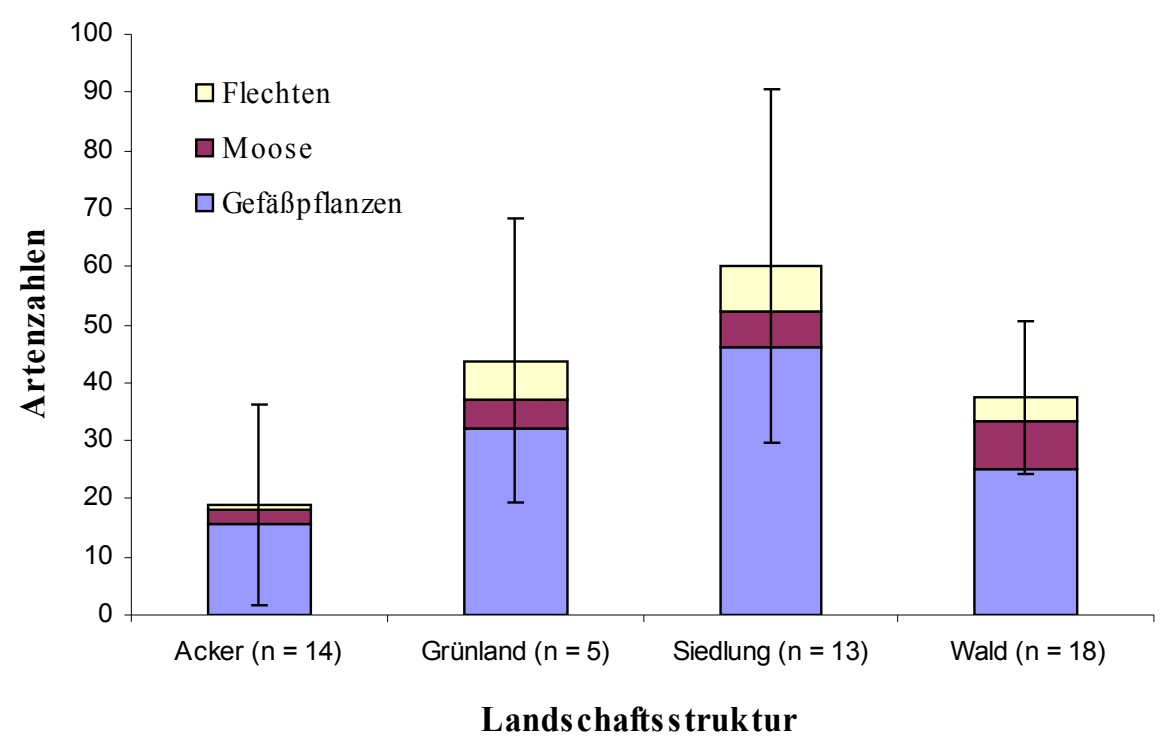

Abbildung 10: Mittlere Artendichten und Standardabweichung der Flechten, Moose und Gefäßpflanzen differenziert nach Landschaftstypen de Fläche A7.

In allen Landschaftstypen sind unter den Gefäßpflanzen die mittleren Artendichten indigener Arten am höchsten (Abb. 11). Während auf den Siedlungsflächen Archaeophyten und Neophyten im Mittel mit 3,5 bzw. 12,3 Gefäßpflanzenarten vertreten sind, macht keine floristische Statusgruppe neben den indigenen Arten auf einem der verbleibenden Landschaftstypen im Mittel mehr als 2 Gefäßpflanzenarten aus. Unter den Neophyten der Siedlungsbereiche waren durchschnittlich 9,9 Gefäßpflanzenarten angepflanzt und nur 2,4 wild wachsend. 


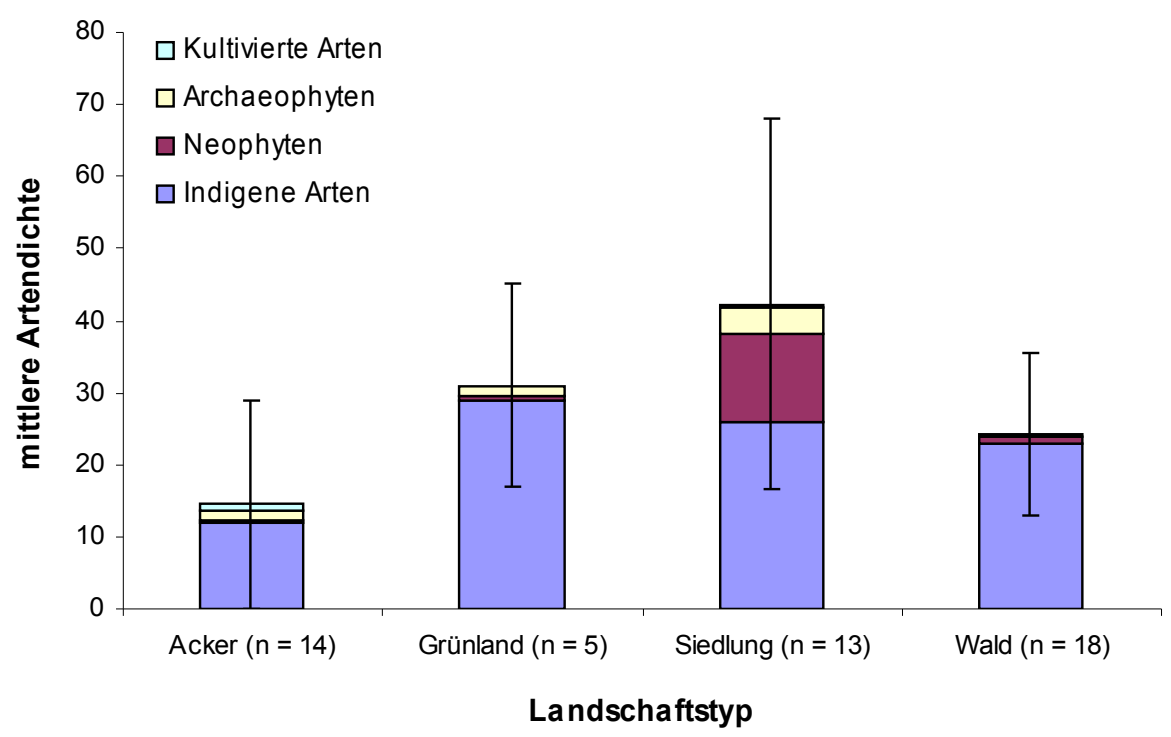

Abbildung 11: Mittlere Artendichten und Standardabweichung der indigenen Arten, Neophyten, Archaeophyten und kultivierten Arten differenziert nach Landschaftstypen der Fläche A7. Berücksichtigt wurden nur Gefäßpflanzen.

Auf keiner der berücksichtigten Flächengrößen sind die Artengruppen untereinander positiv oder negativ korreliert. Das trifft auch nicht zu, wenn man die Artendichten der Gefäßpflanzen mit der Artendichten der vereinigten Artendichten von Flechten und Moosen korreliert.

\subsubsection{Diskussion}

Insgesamt weisen die Artenzahlen eine hohe Variabilität auf mit weiten Bereichen zwischen den Minima und Maxima. Dieser Effekt ist der Art der Stichprobennahme zuzusprechen. Bei absolut zufälliger, also auch nicht stratifizierter, Stichprobenverteilung entspricht die Erfassung von Standorten mit stark divergierenden Artendichten der Erwartung. Je stärker ein Untersuchungsgebiet stratifiziert werden würde, so stärker würden sich die Spannen der Artenzahlen eines Stratums annähern. Eine bereits sehr feine Stratifizierung wäre die Einteilung eines Gebietes nach Vegetationstypen oder Pflanzengesellschaften. So hat Новонм (1998) Vegetationsaufnahmen in Pflanzengesellschaften vorgenommen und gibt für offene Landschaften Mitteleuropas auf einer Fläche von $1 \mathrm{~m}^{2}$ eine durchschnittliche Artenzahl für alle Artengruppen (Samenpflanzen, Farne, Moose und Flechten, was auch den Gesamtartenzahlen der vorliegenden Arbeit entspricht) von 10 Arten an, für eine Fläche von $100 \mathrm{~m}^{2} 25$ Arten. Dies stimmt nicht mit den Ergebnissen der vorliegenden Studie überein. Während die durchschnittliche Artenzahl der kleineren der beiden Flächen über dem von mir ermittelten Wert liegt, ist die von mir ermittelte Durchschnittszahl auf der Fläche A7 größer als die von Новонм (1998: 136) für diese Flächengröße postulierte. Dies liegt daran, dass Новонм (1998) diese Werte nicht explizit auf diesen Flächengrößen gemessen hat. Stattdessen beruhen seine Ergebnisse auf der Berechnung einer Regressionsgeraden für 103 Artenzahl-Fläche-Wertepaare, die er durch Vegetationsaufnahmen in verschiedenen Pflanzengesellschaften ermittelt hat. Die Formel dieser Geraden hat er dann verwendet um die Artenzahlen im logarithmierten Raum auf bestimmten Flächengrößen zu berechnen. Dadurch unterstellt er der Arten-Areal-Beziehung einen linearen Zusammenhang. Dass dies nicht zutreffend ist, wurde inzwischen mehrfach belegt. So wird von den meisten Autoren als 
gesichert angenommen, dass Arten-Areal-Beziehungen am besten von einer Potenzfunktion wiedergegeben werden (Rosenzweig 1995, Crawley \& Harral 2001, Dolnik 2003, Fridley et. al 2005), worauf ich in Kap. 5.1 näher eingehen werde.

Außerdem machen in einer Landschaft, die zu großen Teilen aus Wäldern und Agrarflächen bestehen, die von Новонм (1998) erfassten Pflanzengesellschaften nur einen kleinen Teil aus. Новонм (1998: 161) relativiert seine Ergebnisse hinsichtlich der Artenvielfalt, indem er schreibt, dass aufgrund der genannten Flächenanteile nicht $\mathrm{zu}$ erwarten wäre, dass die von ihm ,,... analysierten Landschaftsausschnitte und Tabellen den Durchschnitt mitteleuropäischer Vegetation repräsentierten“.

In einer Studie aus England, in der ebenfalls mit absolut zufallsverteilter Stichprobenverteilung gearbeitet wurde, sind auf den fraglichen Flächengrößen durchschnittlich 6,38 $\left(1 \mathrm{~m}^{2}\right)$ bzw. 16,23 $\left(100 \mathrm{~m}^{2}\right)$ Gefäßpflanzenarten erfasst worden (CRAWLEY \& HaRRAL, 2001). Allerdings wurden dabei keine gepflanzten Arten berücksichtigt. Um dennoch einen Vergleich mit der vorliegenden Studie zu ermöglichen, müssen dementsprechend die Durchschnittszahlen gepflanzter Arten von denen der gesamten Gefäßpflanzenarten subtrahiert werden. Auch hier sind die durchschnittlichen Artenzahlen, die ich im Rahmen dieser Diplomarbeit ermittelt habe, zum Teil deutlich kleiner als die von Crawley \& Harral (2001). Auf Fläche A1 beträgt der Unterschied 22,5\%. Auf der Fläche A2 sogar 26,6\%. Allerdings ist die Fläche A2 auch $10 \%$ kleiner als die entsprechende Fläche der englischen Studie. Selbiges gilt auch für die Fläche A6, die mit $9 \mathrm{~m}^{2}$ wiederum $10 \%$ kleiner ist als die entsprechende von CRAWLeY \& HaRRAL (2001). Um diesen Flächenunterschied zu berücksichtigen, unterstelle ich, wie von CRAWLEY \& HARRAL (2001) angenommen, der Arten-ArealBeziehung eine Potenzfunktion und berechne die Artenzahlen, die Crawley \& Harral (2001) mit den von ihnen ermittelten Funktionsparametern für die Flächengrößen, die in der vorliegenden Arbeit angewendet wurden, erhalten hätten. Ich reduziere also für die betroffenen Flächengrößen die Artenzahlen der Briten um sie mit den hiesigen Artenzahlen vergleichen zu können. Nach dieser Angleichung beträgt die Differenz der durchschnittlichen Artenzahlen auf der Fläche A2 nur noch 21,1 \%. Auf der Fläche A5 beträgt die Differenz bereits weniger als $10 \%$, während ich auf den beiden größten Flächen durchschnittlich mehr Gefäßpflanzenarten ermittelt habe als CRAWLEY \& HARRAL (2001) (Tab. 5).

Tabelle 5: Vergleich mittlerer Artenzahlen von Gefäßpflanzen auf unterschiedlichen Flächengrößen.

\begin{tabular}{lrrrrr}
\hline Quelle & \multicolumn{5}{c}{ Flächengröße [m } \\
\hline A (Crawley \& Harral 2001) & $0,01 \mathrm{~m}^{2}$ & $0,1 \mathrm{~m}^{2}$ & $1 \mathrm{~m}^{2}$ & $10 \mathrm{~m}^{2}$ & $100 \mathrm{~m}^{2}$ \\
A (Allers) & $0,01 \mathrm{~m}^{2}$ & $0,09 \mathrm{~m}^{2}$ & $1 \mathrm{~m}^{2}$ & $9 \mathrm{~m}^{2}$ & $100 \mathrm{~m}^{2}$ \\
\hline S (Crawley \& Harral 2001) & 3,1 & 4,8 & 6,4 & 10,0 & 16,2 \\
Korrigiertes S (Crawley \& Harral & 3,1 & 4,5 & 6,4 & 9,7 & 16,2 \\
S (Allers) & 2,4 & 3,5 & 5,8 & 10,0 & 24,4 \\
Differenz & $-0,7$ & $-1,3$ & $-0,6$ & 0,0 & 8,1 \\
Differenz [\%] & $-22,5$ & $-26,6$ & $-9,4$ & 0,3 & 50,1 \\
\hline
\end{tabular}

Die durchschnittliche Artenzahl nach Crawley \& Harral (2001) ist auf der Fläche A7 deutlich kleiner, als die von mir ermittelte. Ein Grund für die unterschiedliche Artenzahl könnte ein unterschiedlich hoher regionaler Arten-,,pool“" sein. Allerdings haben Crawley \& HaRral (2001) in ihrer Studie eine durchschnittliche Artenzahl von 801,7 Arten auf $100 \mathrm{~km}^{2}$ ermittelt. Wie bereits in 
der Gebietsbeschreibung (s. Kap. 2.5) angeführt, sind im $126 \mathrm{~km}^{2}$ großen Untersuchungsgebiet der vorliegenden Arbeit 797 Gefäßpflanzenarten nachgewiesen worden (HAEUPLER \& SCHÖNFELDER 1989: 35). Demnach scheint der regionale Artenpool im Untersuchungsgebiet der britischen Studie eher größer zu sein, so dass diese Erklärung für die Trend-Unterschiede der durchschnittlichen Artenzahlen auf größeren Flächengrößen nicht plausibel ist.

Ein weiterer möglicher Lösungsansatz liegt im großen Anteil der Plots im Siedlungsbereich. Zu diesem Schluss komme ich aus mehreren Gründen:

- Sowohl auf öffentlichen als auch auf privaten Flächen werden im Siedlungsbereich oft Pflanzenarten angepflanzt.

- Zusätzlich zu diesen angepflanzten Pflanzenarten kommen meist Wildpflanzen auf den Flächen vor. Der Mensch bringt durch seine gärtnerische Aktivität also Arten in Systeme ein, die dadurch mehr Arten erhalten als es der potentiell natürlichen Vegetation an diesen Standorten entsprechen würde.

- Siedlungsflächen sind kleinräumig vergleichsweise heterogen strukturiert, so dass sich innerhalb eines Plots leicht verschiedene Standorte befinden können. Dadurch haben Siedlungsflächen oft mehr Pflanzenarten als die umliegende Landschaften (vgl. KüHN et al. 2004).

In einer sowohl räumlich als auch methodisch sehr ähnlich angesiedelten Untersuchung in Brandenburg konnten auf allen Flächengrößen durchschnittlich mehr Arten nachgewiesen werden (Dengler \& Allers 2006). Dabei sind zum einen sowohl Flechten und Moose als auch gepflanzte Arten erfasst und zum anderen die gleichen Flächengrößen berücksichtigt worden, was die Daten unmittelbar vergleichbar macht (Tab. 6). Das Untersuchungsgebiet der brandenburgischen Studie lag ca. $320 \mathrm{~km}$ östlich des hiesigen Untersuchungsgebietes nahe der deutsch-polnischen Grenze.

Tabelle 6: Vergleich mittlerer Gesamtartenzahlen auf unterschiedlichen Flächengrößen.

\begin{tabular}{|c|c|c|c|c|c|c|}
\hline \multirow[b]{2}{*}{ Quelle } & \multicolumn{6}{|c|}{ Flächengröße $\left[\mathrm{m}^{2}\right]$} \\
\hline & 0,0009 & 0,01 & 0,09 & 1 & 9 & 100 \\
\hline Dengler \& Allers 2006 & 2,7 & 4,2 & 6,2 & 11 & 18,1 & 42,9 \\
\hline Allers & 2,4 & 3,5 & 5,1 & 8,5 & 15,2 & 38,7 \\
\hline Differenz & $-0,3$ & $-0,7$ & $-1,1$ & $-2,5$ & $-2,9$ & $-4,2$ \\
\hline Differenz [\%] & $-11,1$ & $-16,7$ & $-17,7$ & $-22,7$ & $-16,0$ & $-9,8$ \\
\hline
\end{tabular}

Für diese Unterschiede gibt es mehrere denkbare Erklärungen. In Frage kommen zum Beispiel eine strukturreichere Landschaft in Brandenburg oder eine extensivere Landwirtschaft, was erhöhte Artendichten auf Agrarflächen fördert. Auf diesen Flächen ist die Artenzahl im Rahmen dieser Untersuchung am geringsten (Abb. 10). Außerdem könnte der regionale Artenpool in Brandenburg höher sein als im Nordosten Niedersachsens. Arten mit eher kontinentalem Verbreitungsschwerpunkt sind hier durch das stark subkontinental geprägte Klima stärker verbreitet.

Eine Vergleichsmöglichkeit der mittleren Artendichten der Waldplots bieten Dupré et al. (2002), die in Öland und Uppland in Schweden 22 bzw. 14 Plots mit geschachtelten Serien von Subplots ausgewertet haben. In beiden Fällen wurden wieder nur Gefäßpflanzenarten aufgenommen. Ein Vergleich der Artendichten in Laubwäldern des hiesigen Untersuchungsgebietes zeigt eine deutlich niedrigere mittlere Artenzahl. Auf der Flächengröße A5 haben DupRÉ et al. (2002) für Uppland 9,1 
und für Öland sogar 14,4 Gefäßpflanzenarten ermittelt. Von den von mir untersuchten Plots auf Waldflächen befanden sich fünf Plots in reinen Laubwäldern. Auf diesen habe ich auf der entsprechenden Flächengröße 6,7 Gefäßpflanzenarten registriert. Das liegt daran, dass drei der fünf von mir erfassten Laubwälder $\mathrm{zu}$ artenarmen Wäldern des Verbandes Luzulo-Fagion (Drahtschmielen- und Hainsimsen-Buchen-Wälder) gehören (Preising et al. 2003). Dies sind Verbände, die sich durch dominierende Bestände von Fagus sylvatica und Carpinus betulus und eine sehr artenarme Krautschicht auszeichnen. Da dieser Mittelwert auf einer Anzahl von nur fünf Plots beruht ist seine Aussagekraft allerdings auch gering, da er Extremfälle nicht kompensieren kann. Zwei der Plots wiesen auf einem Quadratmeter sogar nur 2 bzw. 2,25 Arten auf. Das wirkt sich stark auf die mittleren Artendichten der fünf Plots aus.

DieKMANN et al. (2007) haben auf der Flächengröße A5 durchschnittlich sogar nur drei Gefäßpflanzenarten ermittelt. Dieser extrem niedrige Wert kann zum Teil durch den geringeren regionalen Artenpool erklärt werden. Laut HAeupler \& SchöNFelder (1989) kommen auf dem Messtischblatt innerhalb dessen die Studie von Diekmann et al. (2007) durchgeführt wurde 437 Gefäßpflanzenarten vor, während im Gebiet der hiesigen Untersuchung bislang 797 Gefäßpflanzenarten nachgewiesen wurden. Trotzdem wirken diese Artenzahlen sehr niedrig, da Chiarucci et al. (2006) auf Grünlandflächen ebenfalls in der Nähe von Bremen durchschnittliche Gefäßpflanzenartenzahlen ermittelt haben, die den in dieser Studie erfassten sehr ähnlich sind. Über alle sechs vergleichbaren Flächengrößen sind die mittleren Gefäßpflanzenartenzahlen der vorliegenden Studien nur um 4,1 \% größer als die von CHIARUCCI ert al. (2006), während die gleiche mittlere Artenzahl auf den Waldflächen der hiesigen Studie über die drei vergleichbaren Flächengrößen gemittelt 117,6 \% größer ist als die von DiEKMANN et al. (2007) ermittelte.

Obwohl deren Anteil an den absoluten Artenzahlen sehr hoch liegt, konnten auf den Plots durchschnittlich nur wenig Neophyten und Archaeophyten nachgewiesen werden. Offensichtlich kamen die meisten der Arten nur auf wenigen Plots vor (s. Kap. 6). Das Vorkommen der Neophyten ist dabei besonders an gärtnerische Aktivitäten gebunden. Die meisten von ihnen fanden sich im Siedlungsbereich, wo sie zum überwiegenden Teil angepflanzt vorkamen. Dass die Neophyten auch ohne den Anteil gepflanzter Arten im Siedlungsbereich durchschnittlich die meisten Arten aufweisen, ist ein bekanntes Phänomen und begründet sich in der allgemein höheren Artendichte auf Siedlungsflächen (KüHN et al. 2004, WANIA et al. 2006). Tatsächlich lässt sich auf der Fläche A7 ein positiver Zusammenhang zwischen der Artendichte der Neophyten und der Gesamtartenzahl feststellen (Abb. 12). 


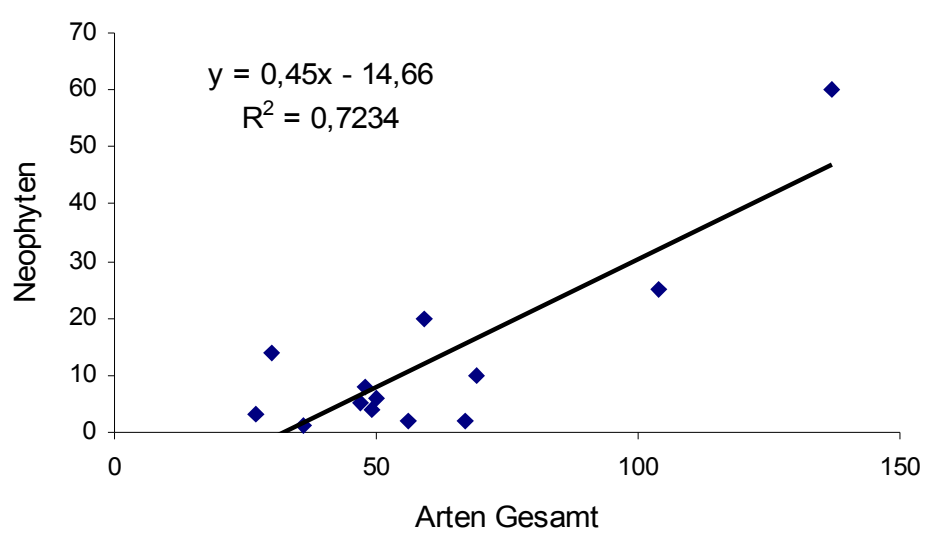

Abbildung 12: Artendichte der Neopyhten in Abhängigkeit zur Gesamtartendichte der Fläche A7.

Die maximale Artenzahl der vorliegenden Studie auf $100 \mathrm{~m}^{2}$ ist sehr hoch und vergleichbar mit der einer Aufnahmefläche gleicher Größe in einer Trockenrasengesellschaft. Trockenrasen gehören zu den artenreichsten Vegetationstypen Europas, was sich nicht nur auf das gesamte Arteninventar bezieht, sondern auch auf die Artendichte auf kleineren Flächengrößen (Новонм 1998, DengleR 2005). So geben Dengler et al. (2006) für einen estnischen Bestand der Gesellschaft CrepidoAllietum eine maximale Artenzahl von 140 Arten auf $100 \mathrm{~m}^{2}$ ermittelt. Das ist selbst für die Trockenrasen in Europa ein sehr hoher Wert (DengLer et al. 2006). Diese Artendichte ist begünstigt durch die hohe Artenzahl von Flechten und Moosen. Nun lässt sich die Artendichte eines intensiv genutzten und gestalteten Ziergartens nicht gut mit der eines natürlichen Trockenrasens vergleichen. Allerdings mag es verdeutlichen, dass Artendichte und Artenvielfalt auf Siedlungsflächen enorme Ausmaße annehmen können und somit bei Biodiversitätsforschungen Siedlungsbereiche nicht vernachlässigt werden dürfen.

Dieser hohe Wert auf dieser Flächengröße entspricht auch den Ergebnissen von Dengler \& Allers (2006). Es gibt mehrere Effekte, die diese Artendichte bedingen können. Generell ist der Anstieg einer Artendichte bis zu einm bestimmten $\mathrm{Ma} ß$ dadurch zu erklären, dass die durchschnittliche Wahrscheinlichkeit seltene Arten zu erfassen mit der Anzahl erfasster Pflanzenindividuen wächst. Räumlich angehäufte Vorkommen bestimmter Arten werden eher erfasst, umso größer die Aufnahmefläche wird (vgl. MAY 1975). Die im Vergleich zur Fläche A6 besonders hohe Artenzahl der Fläche A7 wird dabei zusätzlich durch den bereits erwähnten Effekt der zufälligen Stichprobenverteilung begünstigt.

Dass die Artendichten der Gefäßpflanzen nicht mit den Artendichten der Nicht-Gefäßpflanzen postulieren auch Pнaro et al. (1999). Zwar finden sie eine positive Korrelation der Artendichten von Moosen und Farnen. Letztere stellen aber nur einen Teil der Gefäßpflanzenarten und können die Informationsgehalt von Flechten und Moosen nicht ersetzen. Im Untersuchungsgebiet der vorliegenden Studie ist der Anteil an Farnen zusätzlich gering und nur durch wenige Arten repräsentiert. 


\subsection{Verteilung der Artenzahlen}

In diesem Abschnitt soll die Verteilung der Artenzahlen anhand spezieller Kenngrößen, wie der Schiefe und der Kurtosis (auch Wölbung oder Exzess genannt) untersucht werden. Es wird der Grad der Normalverteilung getestet und wie dieser sich bei einer Transformation der Artenzahlen ändert.

\subsubsection{Ergebnisse}

Während die Tatsache, dass sich arithmetisches Mittel und Median der Gesamtartenzahlen und Gefäßpflanzenarten ähnlich sind, für eine Gleichverteilung zumindest dieser Artenzahlen spricht, weist die Schiefe in fast allen Fällen auf eine schiefe Verteilung der Artenzahlen hin (Tab. 7). Sowohl Schiefe als auch Kurtosis der Gesamtartenzahlen steigen mit zunehmender Flächengröße an. Die Schiefe der Gefäßpflanzen schwankt auf allen Flächengrößen zwischen 0,7 und 1. Die Schiefe der Moose und Flechten hingegen fällt bei zunehmender Flächengröße. Steigende und fallende Tendenzen und Maxima der Kurtosis sind bei den Artengruppen auf den gleichen Flächengrößen zu finden wie bei der Schiefe. Die Werte der Kurtosis sind in den meisten Fällen positiv, die Verteilungen also steilgipflig. Lediglich einige der Gruppierungen mit den höchsten durchschnittlichen Artenzahlen (Gesamtarten, Gefäßpflanzen und indigene Arten) haben auf manchen Flächengrößen einen negativen Kurtosiswert und sind damit flachgipflig verteilt. Während die Kurtosis bei drei der vier untersuchten floristischen Statusgruppen bei zunehmender Flächengröße einem gleich bleibendem Trend folgt, fällt bei den drei Artengruppen auf, dass der Durchschnittswert der Kurtosis der Fläche A6 sich deutlich von dem der Fläche A7 abhebt. Die Schiefe wird bei den Flechten und Moosen sowie den Archaeophyten und kultivierten Arten mit zunehmender Flächengröße kleiner. Bei den Gesamtarten und den Gefäßpflanzen einerseits und den indigenen Arten und Neophyten andererseits wird sie hingegen ein geringfügig größer.

Tabelle 7: Schiefe (obere Tab.) und Kurtosis (untere Tab.) der Gesamtarten-, Gefäßpflanzenarten-, Flechtenarten- und Moosartenzahlen sowie der floristischen Gruppen (mit indigen Arten = Ind., Neophyten $=$ Neo., Archaephythen $=$ Arch. und kultivierten Pflanzen $=$ Kult.) auf den unterschiedlichen Flächengrößen.

\begin{tabular}{lllllllll}
\hline Fächengröße $\left[\mathbf{m}^{2}\right]$ & Gesamt & Gefäßpfl. & Moose & Flechten & Ind. & Neo. & Arch. & Kul. \\
\hline $0,0001(\mathrm{n}=200)$ & 0,5 & 0,7 & 2,3 & 9,9 & 0,5 & 5,3 & 14,1 & 2,9 \\
$0,0009(\mathrm{n}=200)$ & 0,5 & 0,8 & 2,4 & 6,9 & 0,4 & 6,0 & 8,0 & 2,4 \\
$0,01(\mathrm{n}=200)$ & 0,5 & 0,7 & 1,6 & 6,3 & 0,4 & 4,8 & 4,9 & 2,0 \\
$0,09(\mathrm{n}=200)$ & 0,6 & 0,7 & 1,3 & 5,1 & 0,5 & 3,8 & 3,5 & 1,8 \\
$1(\mathrm{n}=200)$ & 0,7 & 0,8 & 1,0 & 3,0 & 0,7 & 5,5 & 2,5 & 2,2 \\
$9(\mathrm{n}=200)$ & 0,9 & 1,0 & 1,0 & 2,0 & 0,5 & 5,3 & 1,9 & 2,0 \\
$100(\mathrm{n}=50)$ & 1,4 & 0,8 & 0,8 & 1,8 & 0,1 & 4,7 & 1,1 & 1,6 \\
\hline & & & & & & & & \\
\hline Fächengröße $\left[\mathbf{m}^{2}\right]$ & Gesamt & Gefäßpfl. & Moose & Flechten & Ind. & Neo. & Arch. & Kul. \\
\hline $0,0001(\mathrm{n}=200)$ & $-0,5$ & 0,3 & 5,8 & 97,5 & $-0,7$ & 34,4 & 200,0 & 6,4 \\
$0,0009(\mathrm{n}=200)$ & $-0,5$ & 0,7 & 6,6 & 54,1 & $-0,9$ & 47,7 & 63,3 & 3,6 \\
$0,01(\mathrm{n}=200)$ & $-0,7$ & $-0,2$ & 2,4 & 48,0 & $-1,0$ & 29,8 & 25,6 & 2,1 \\
$0,09(\mathrm{n}=200)$ & $-0,3$ & $-0,1$ & 1,1 & 27,1 & $-0,6$ & 18,0 & 12,2 & 1,3 \\
$1(\mathrm{n}=200)$ & 0,1 & 0,1 & 0,5 & 8,7 & 0,2 & 37,2 & 5,3 & 4,3 \\
$9(\mathrm{n}=200)$ & 1,1 & 1,8 & 1,6 & 4,2 & 0,1 & 34,4 & 2,8 & 3,3 \\
$100(\mathrm{n}=50)$ & 3,7 & $-0,5$ & 0,5 & 6,1 & $-0,7$ & 25,7 & 0,0 & 1,5 \\
\hline
\end{tabular}


Eine Normalverteilung kann man höchstens bei den Gesamtarten oder den Gefäßpflanzenarten unterstellen. Der Kolmogorov-Smirnow-Test für die Gesamtarten der Fläche A6 ergibt ein Signifikanzniveau von $\mathrm{p}>0,20$, weshalb die Abweichung von der Normalverteilung nicht signifikant ist (Abb. 13, vgl. Dутнам 2003).

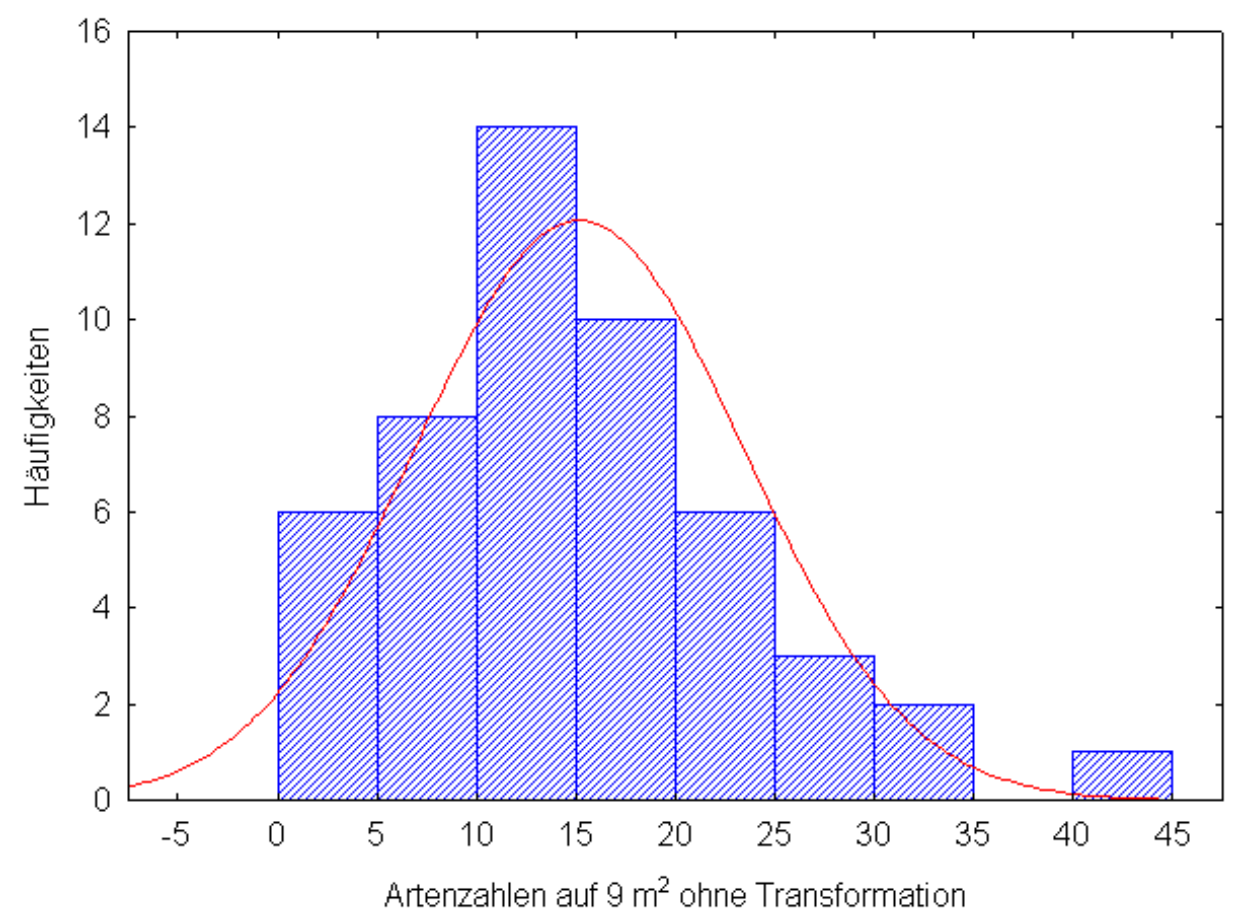

Abbildung 13: Histogramm der absoluten Artenzahlen der Fläche A6 mit Normalverteilungskurve.

Die Verteilung ist allerdings offensichtlich rechtsschief. Deshalb führe ich eine logarithmische Transformation der Artenzahlen durch, die laut LozÁN \& KAusch (2004) oft eine bessere Annäherung an eine Normalverteilung mit sich bringt. Der Kolmogorov-Smirnow-Test zeigt hier allerdings das gleiche Signifikanzniveau mit $p>0,20$. Dadurch weist zwar auch die logarithmierten Artenzahlen keine signifikante Abweichung von der Normalverteilung auf. Das Histogramm für diese transfomierten Daten zeigt aber eine deutlich linksschiefe Verteilung (Abb. 14). 


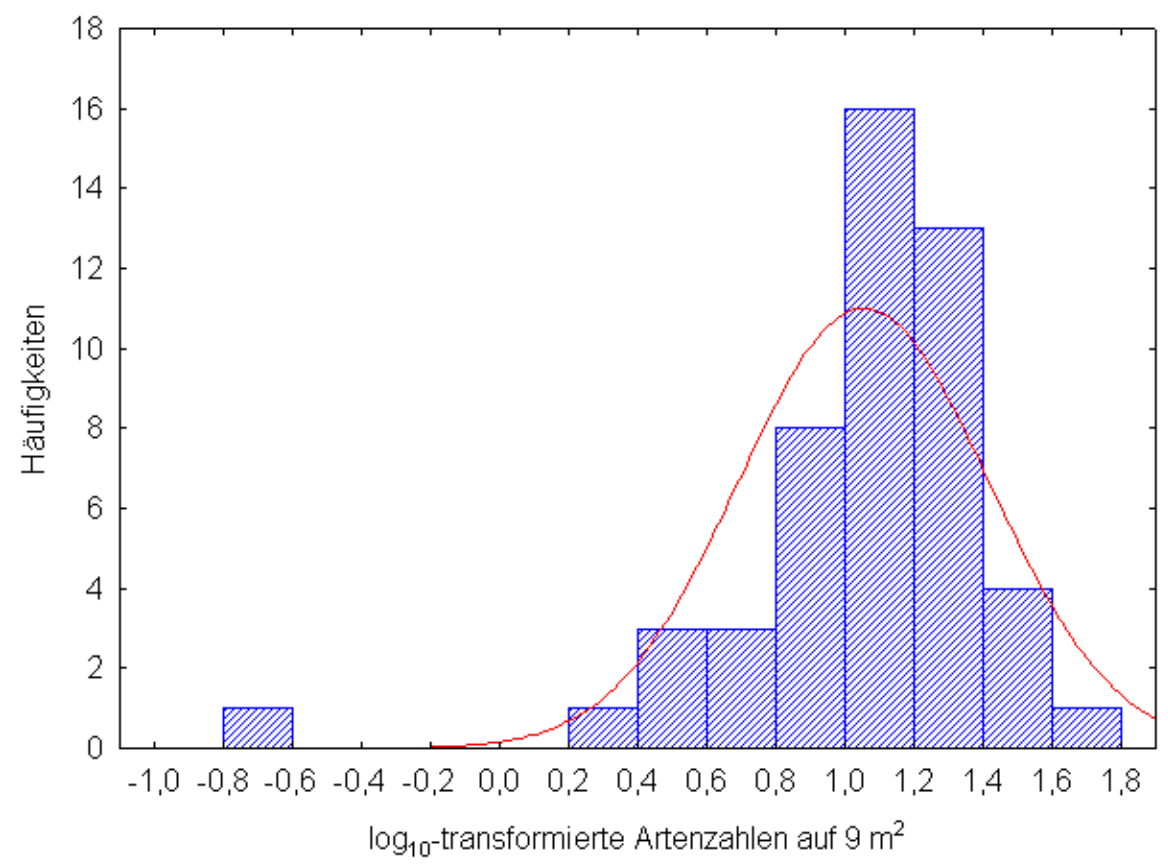

Abbildung 14: Histogramm der logarithmierten Artenzahlen der Fläche A6 mit Normalverteilungskurve.

Da die Verteilung der absoluten Artenzahlen linksschief ist und die der logarithmierten Artenzahlen rechtsschief, teste ich eine weitere Möglichkeit der Daten-Transformation: die Arcus-sinusTransformation. Das Ergebnis ist aber auch hier das gleiche. Das Signifikanzniveau liegt auch hier bei $p>0,20$, weshalb keine signifikante Abweichung von der Normalverteilung angenommen werden kann. Das Histogramm zeigt eine leicht bessere Anpassung der Daten an eine Normalverteilung (Abb. 15).

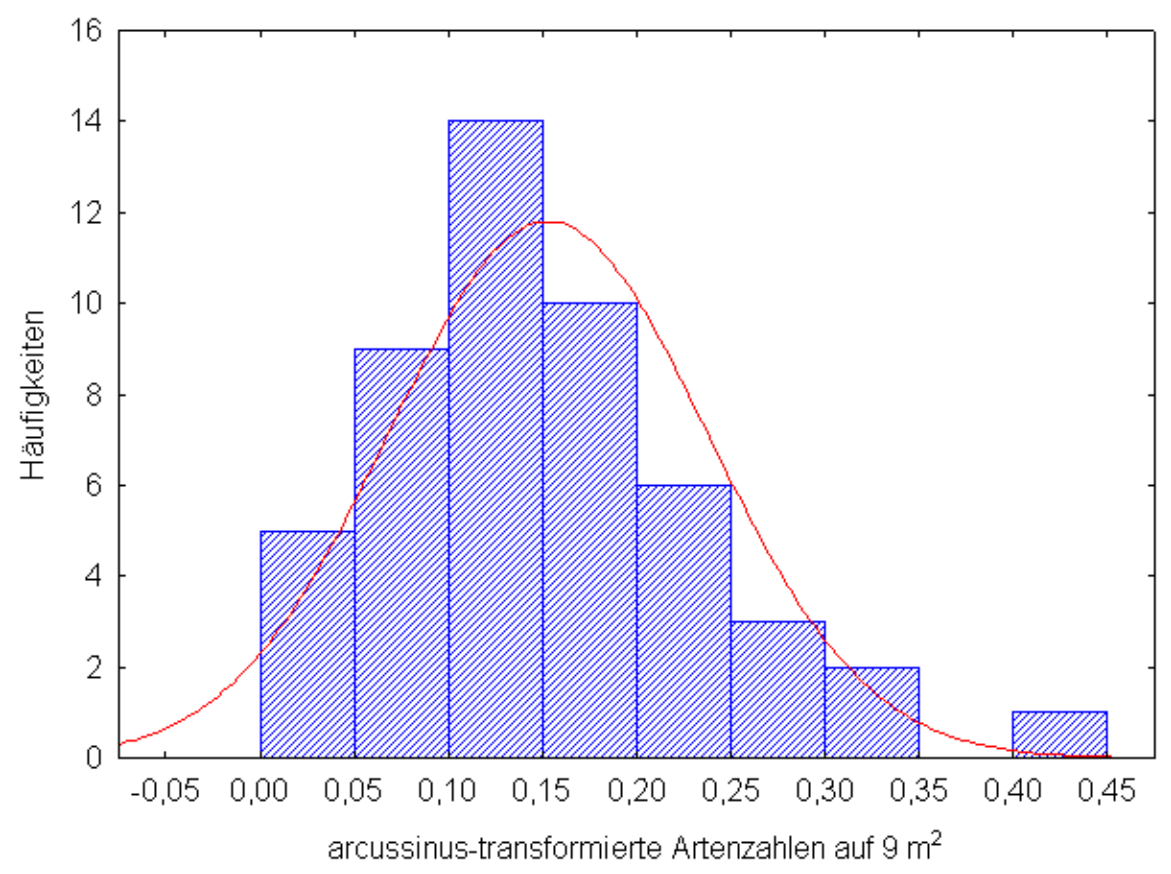

Abbildung 15: Histogramm der arcus-sinus-transformierten Artenzahlen der Fläche A6 mit Normalverteilungskurve.

Während die Artenzahlen der Gefäßpflanzen und Moose nicht signifikant von einer 
Normalverteilung abweichen (in beiden ist $\mathrm{p}>0,20$ ), kann für die Artenzahlen der Flechten mit $\mathrm{p}<0,05$ keine Normalverteilung angenommen werden. Diese Feststellung wird durch das Histogramm in Abb. 16 bestätigt. Auch in diesem Fall ermöglicht eine logarithmische Transformation keine bessere Annäherung an eine Normalverteilung.

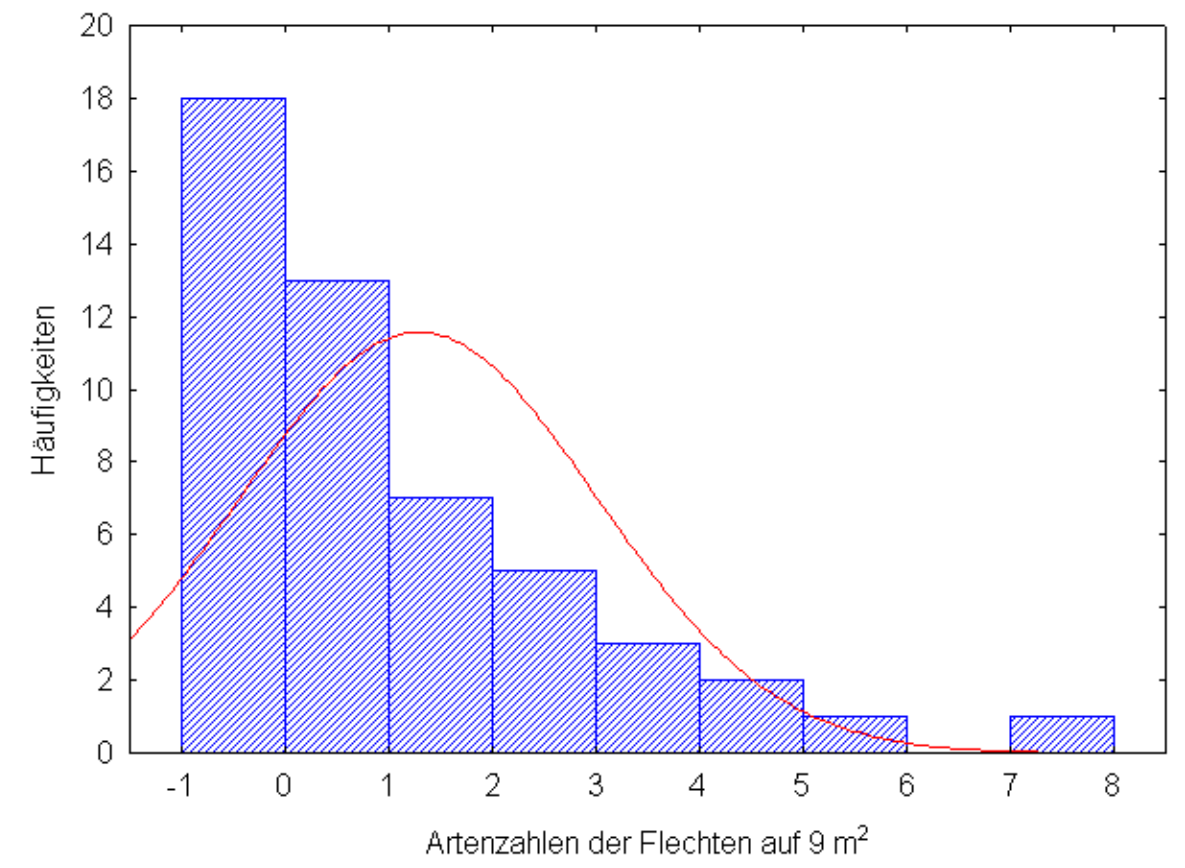

Abbildung 16: Histogramm der absoluten Artenzahlen der Flechten der Fläche A6 mit Normalverteilungskurve.

Außer bei den indigenen Arten können auch bei den floristischen Statusgruppen keine Normalverteilungen angenommen werden.

Die Werte der Kurtosis der Gesamtarten und Gefäßpflanzen liegen bei allen Flächengrößen relativ nahe an Null. Neben diesen Artengruppen zeigen lediglich die Kurtosiswerte der indigenen Arten negative Werte auf. Alle anderen Werte weisen auf steilgipflige Verläufe der Kurven hin.

Flechten und Moose zeigen eine relativ heterogene Verteilung. Bei den Flechten, bei denen das arithmetische Mittel auf allen Flächengrößen kleiner ist als die Standardabweichung, ist der Median nur auf der Fläche A7 größer als Null. Ähnliches gilt für die Artenzahlen der Moose. Das arithmetische Mittel ist nur bei den Flächen A6 und A7 größer als die Standardabweichung. Die Schiefe ist bei den drei größten Flächen eins oder kleiner.

Die Artenzahlen der Gefäßpflanzen zeigen eine homogenere Verteilung. Die Schiefe nimmt auf der Fläche A6 ihren vergleichsweise geringen Maximalwert von eins an. Das arithmetische Mittel ist für alle Flächengrößen größer als die Standardabweichung und der Median mindestens auch bei der Fläche A1 eins.

\subsubsection{Diskussion}

Artenverteilungen zeigen in der Regel einen linksschiefen Verlauf, weshalb in der Praxis die Artenzahlen (z. B. logarithmisch) transformiert werden müssen, um eine möglichst perfekte Normalverteilung zu erreichen (Dутнам 2003). Dass die Artenzahlen bei der vorliegenden 
Untersuchung bei einer logarithmischen Transformation einen rechtsschiefen Verlauf zeigen, ist dagegen eher ungewöhnlich. Wahrscheinlich ist die Abweichung der absoluten Artenzahlen im Sinne der linksschiefen Verteilung nicht stark genug, so dass sich die effektive logarithmische Transformation zu heftig auswirkt. Die bessere Annäherung der Artenzahlen an eine Normalvertielung durch eine Arcus-sinus-Transformation ist dadurch bedingt, dass diese die Artenzahlen weniger stark verändert. Trotzdem konnte im Gegensatz dazu auch durch die logarithmische Transformation der Artenzahlen der Flechten deren Verteilung nicht besser an eine Normalverteilung angenähert werden. Der Grad der Schiefe war in diesem Falle möglicherweise zu stark.

Dутнам (2003) folgend fallen bei einer perfekten Normalverteilung $99 \%$ der beobachteten Werte in den Bereich von

$$
\varnothing \pm 2,576 \cdot S D
$$

$\varnothing$ das arithmetische Mittel und SD die Standardabweichung darstellt. Dieser mathematische Zusammenhang kann genutzt werden, um die Ergebnisse dieser Arbeit mit anderen vegetationskundlichen Daten zu vergleichen. Berechnet man diese Bereiche für die Gesamtarten, Gefäßpflanzen, Moose und Flechten für zwei Trockenrasenverbände von Bосн (2005), zeigt sich, dass zumindest die Gesamtartenzahlen beider Verbände relativ gut einer Normalverteilung entsprechen. Beim Crepido-Allietum sind die Gesamtartenzahlen lediglich etwas steiler, da sein Minimum und Maximum eine kleinere Spanne umfassen, als es bei einer Normalverteilung der Fall wäre. Bei den verschiedenen Artengruppen weichen die oberen und unteren Grenzen der unterstellten Normalverteilung mehr von den von Восн (2005) ermittelten ab, so dass hier größere Abweichungen von einer Normalverteilung angenommen werden können. Dabei ist die Abweichung bei den Moosen größer als bei den Gefäßpflanzen und bei den Flechten wiederum noch größer als bei den Moosen (Tab. 8). Das entspricht weitestgehend den im Rahmen dieser Arbeit ermittelten Ergebnissen, so dass davon ausgegangen werden kann, dass Gefäßpflanzen und Moose auf wenigen Standorten eine verhältnismäßig geringe, auf vielen Standorten eine mittlere und auf wenigen Standorten eine verhältnismäßig hohe Artendichte haben. Die Flechten hingegen sind auf vielen Standorten mit nur geringen Artendichten vertreten und nur auf wenigen Standorten mit hohen Artendichten. Das stimmt zumindest dann, wenn man davon ausgeht, dass mit den Plots die Artendichten der Artengruppen repräsentativ erfasst wurden, worauf ich im Kapitel 4.4 näher eingehen werde. 
Tabelle 8: Arithmetisches Mittel, Standardabweichung, Minimum und Maximum zweier Trockenrasenverbände (Восн 2005) sowie die Bereiche, die $99 \%$ der Werte bei einer unterstellten Normalverteilung enthalten würden.

\begin{tabular}{lrrrrrr}
\hline \multicolumn{7}{c}{ Festucetum polesicae } \\
& $\varnothing$ & $S D$ & Min & Max & $\varnothing-S D_{99 \%}$ & $\varnothing+S D_{99 \%}$ \\
\hline Gesamtarten & 23,1 & 5,6 & 9,0 & 37,0 & 8,7 & 37,5 \\
Gefäßpflanzen & 9,7 & 4,7 & 3,0 & 23,0 & $-2,4$ & 21,8 \\
Moose & 5,8 & 1,8 & 1,0 & 9,0 & 1,2 & 10,4 \\
Flechten & 7,7 & 3,2 & 1,0 & 14,0 & $-0,5$ & 15,9 \\
\hline
\end{tabular}

\begin{tabular}{|c|c|c|c|c|c|c|}
\hline & \multicolumn{4}{|c|}{ Crepido-Allietum } & \multirow[b]{2}{*}{$\varnothing-S D_{99 \%}$} & \multirow[b]{2}{*}{$\varnothing+S D_{99 \%}$} \\
\hline & $\varnothing$ & $S D$ & Min & $\operatorname{Max}$ & & \\
\hline Gesamtarten & 47,7 & 11,4 & 23,0 & 71,0 & 18,3 & 77,1 \\
\hline Gefäßpflanzen & 21,4 & 5,4 & 11,0 & 36,0 & 7,5 & 35,3 \\
\hline Moose & 14,1 & 5,6 & 4,0 & 27,0 & $-0,3$ & 28,5 \\
\hline Flechten & 11,4 & 5,7 & 1,0 & 23,0 & $-3,3$ & 26,1 \\
\hline
\end{tabular}

\subsection{Mittlere Artenanteile}

Während sich das vorherige Kapitel mit den Artendichten bestimmter Artengruppen auf bestimmten Flächengrößen beschäftigt hat, geht es in diesem Abschnitt um die Anteile unterscheidbarer Artengruppen an der mittleren Gesamtartendichte. Es werden die Anteile der verschiedenen Artengruppen sowie der unterschiedlichen floristischen Statusgruppen jeweils insgesamt als auch differenziert nach den Landschaftstypen erörtert werden.

\subsubsection{Ergebnisse}

Der prozentuale Anteil der mittleren Artenzahlen der berücksichtigten Artengruppen variiert innerhalb der verschiedenen Flächengrößen. Der Anteil der Moose an der Artendichte insgesamt nimmt nach anfänglichem Anstieg mit zunehmender Flächengröße ab. Er erreicht dabei ein Maximum von ca. $20 \%$ auf der Fläche A4. Der mittlere Artenanteil der Flechten steigt mit zunehmender Flächengröße von weniger als $1 \%$ auf der kleinsten auf mehr als $11 \%$ auf der Fläche A7 an (Abb. 17). Der Anteil der Gefäßpflanzen sinkt zwar stetig, aber geringfügig. Die größeren Schwankungen gibt es bei den Flechten und Moosen. Was die Moose an Anteilen verlieren, kommt bei den Flechten hinzu. Insgesamt kann man sagen, dass auch zur größten untersuchten Fläche kein Einpendeln, sprich keine Annäherung an eine endgültige Verteilung, der mittleren Artendichten zu erkennen ist. 


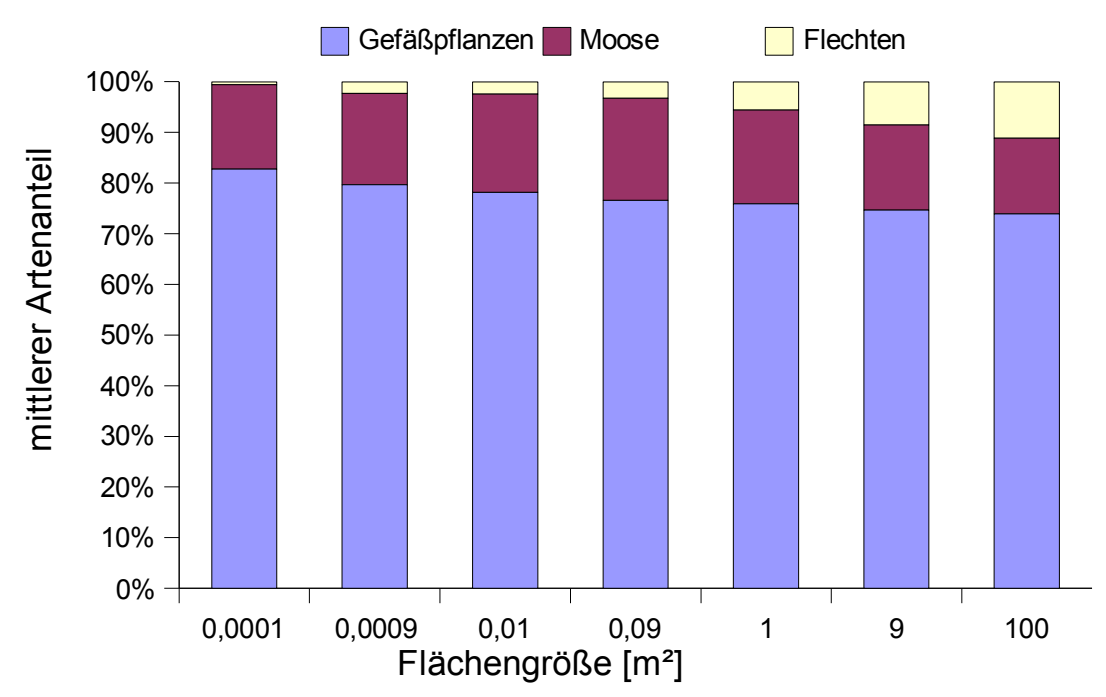

Abbildung 17: Mittlerer Artenanteil der Flechten, Moose und Gefäßpflanzen in Abhängigkeit zur Flächengröße.

Die Artenzahlen der Flechten und Moose werden dabei durch das Vorkommen bestimmter Substrate beeinflusst. Die Wahrscheinlichkeit, dass die Substrat-Straten auf einer Fläche vertreten sind, steigt mit der Größe der Fläche. So steigen die prozentualen Anteile der mittleren Artenzahlen epiphytischer, epixyler und epilithischer Arten von weniger als $5 \%$ auf der Fläche A1 auf fast $60 \%$ auf der Fläche A7. Diese Anteile beziehen sich lediglich auf die Artengruppen Flechten und Moose. Da nur Arten dieser Gruppen in den Substrat-Straten wuchsen, können Gefäßpflanzen bei dieser Betrachtung vernachlässigt werden. Den größten Teil machen auf allen Flächengrößen die Moose auf dem Erdboden aus.

Der mittlere Anteil der Flechten ist auf der Fläche A7 am größten auf den Grünlandflächen (Abb. 19). Gleichzeitig war hier der mittlere Anteil von Moosarten auf der Flächen A1 am größten, der sich aber zur größten Flächengröße hin stetig verringert. Auf den Waldflächen war der mittlere Anteil der Moose auf der Fläche A7 verglichen mit den übrigen unterschiedenen Landschaftstypen am größten, während er auf der Fläche A1 deutlich kleiner war als auf den Siedlungsflächen oder im Grünland. Der Anteil der Flechten ist ausgenommen auf der größten Fläche auf allen Flächengrößen am höchsten auf den Siedlungsflächen. Da der mittlere Anteil der Moose auf den Waldflächen doppelt so hoch ist wie bei den übrigen Landschaftstypen, ist auf den Waldflächen der Anteil von Flechten und Moosen insgesamt am größten. Während die mittleren Anteile der Artendichten von Flechten und Moosen auf den Grünlandflächen eine konkave und auf den Siedlungsflächen eine konvexe Verteilung aufweisen, steigen sie auf den Waldflächen stetig an. 

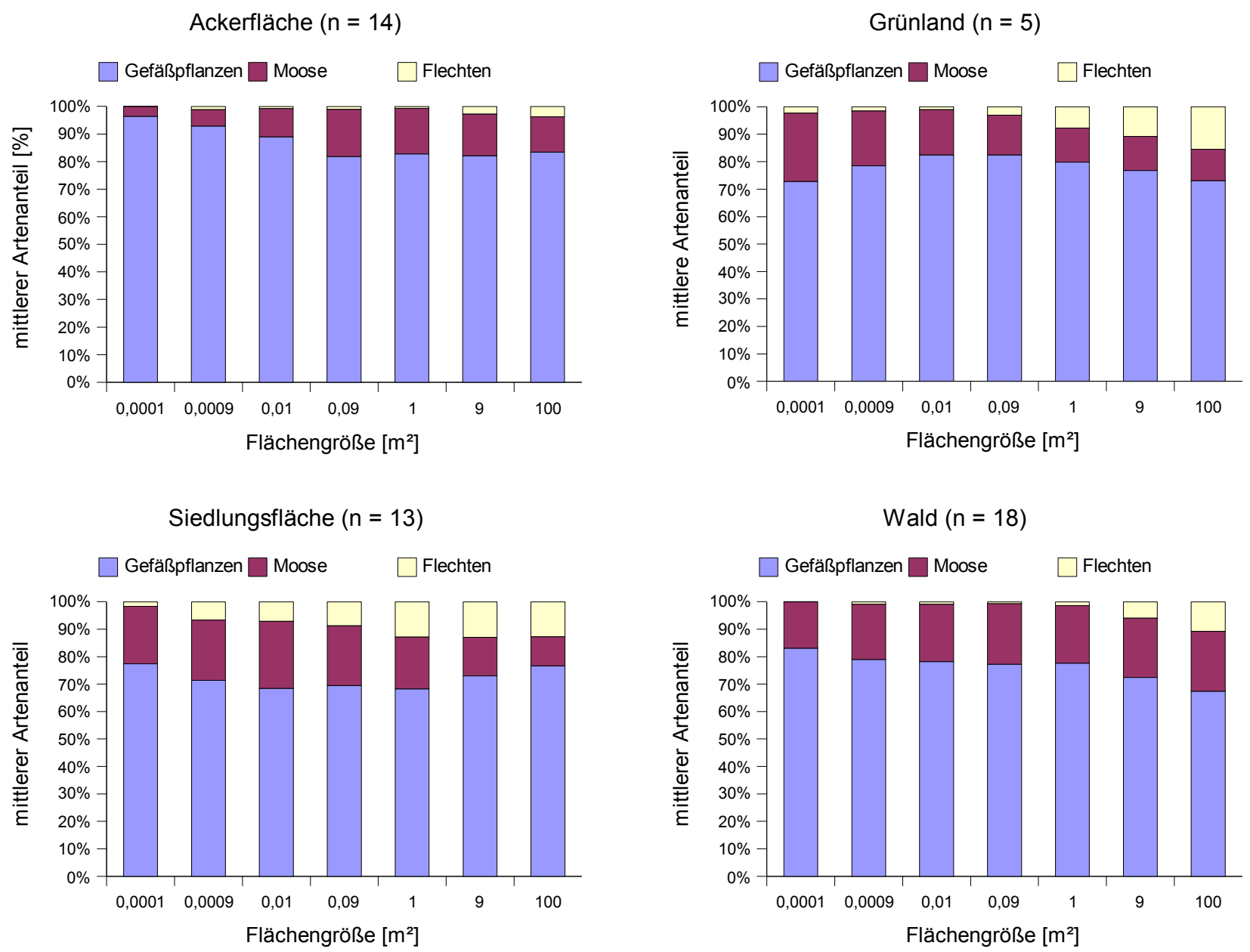

Abbildung 19: Mittlere Artenanteile der Artengruppen differenziert nach den verschiedenen Landschaftstypen in Abhängigkeit zur Flächengröße.

Der mittlere Anteil der Flechten ist auf der Fläche A7 am größten auf den Grünlandflächen (Abb. 19). Gleichzeitig war hier der mittlere Anteil von Moosarten auf der Flächen Al am größten, der sich aber zur größten Flächengröße hin stetig verringert. Auf den Waldflächen war der mittlere Anteil der Moose auf der Fläche A7 verglichen mit den übrigen unterschiedenen Landschaftstypen am größten, während er auf der Fläche A1 deutlich kleiner war als auf den Siedlungsflächen oder im Grünland. Der Anteil der Flechten ist ausgenommen auf der größten Fläche auf allen Flächengrößen am höchsten auf den Siedlungsflächen. Da der mittlere Anteil der Moose auf den Waldflächen doppelt so hoch ist wie bei den übrigen Landschaftstypen, ist auf den Waldflächen der Anteil von Flechten und Moosen insgesamt am größten. Während die mittleren Anteile der Artendichten von Flechten und Moosen auf den Grünlandflächen eine konkave und auf den Siedlungsflächen eine konvexe Verteilung aufweisen, steigen sie auf den Waldflächen stetig an. 


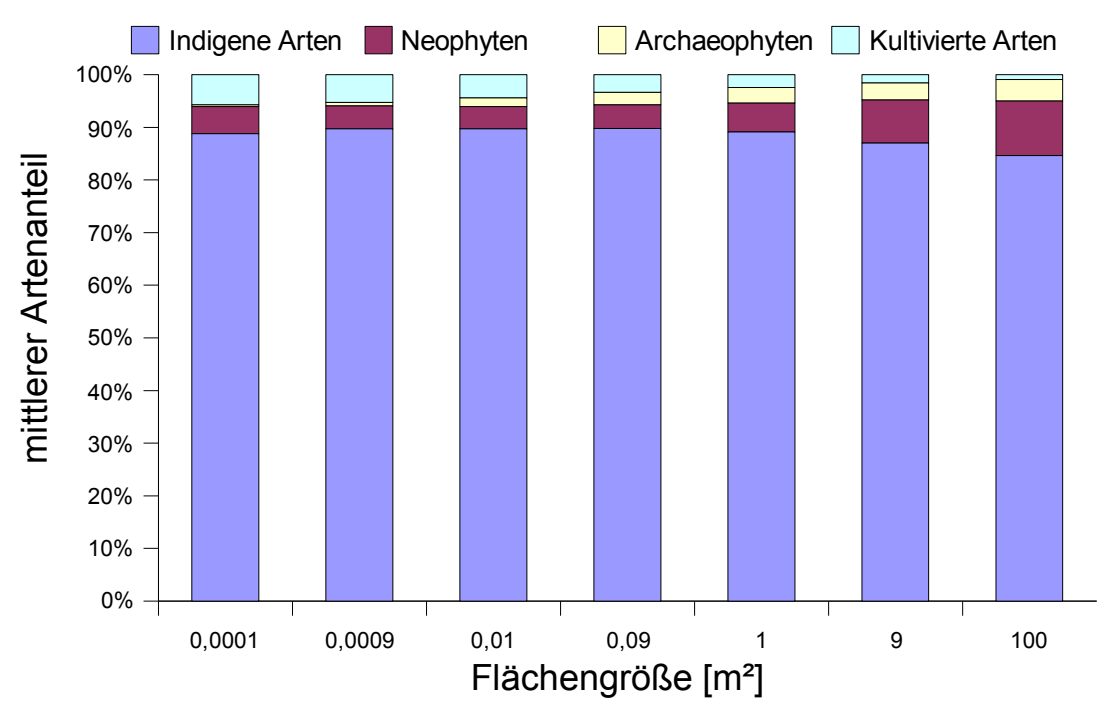

Abbildung 20: Mittlerer Anteil der floristischen Statusgruppen (s. Kap.

3.3 für Definitionen) in Abhängigkeit zur Flächengröße.

Betrachtet man die floristischen Statusgruppen, fällt auf, dass kultivierte Arten auf der Fläche A1 einen Anteil von mehr als $6 \%$ haben, während der Anteil auf der größten Fläche unterhalb eines Prozentes liegt. Während die Anteile der kultivierten Arten mit zunehmender Flächengröße zurückgehen, erhöhen sich die Anteile der Archaeophyten und Neophyten (Abb. 20). Die Anteile der Neophyten sind dabei auf den kleinen Flächen relativ konstant und fangen erst mit der Fläche A5 an deutlich zu steigen. Einen konsistenten Anstieg von der kleinsten zur größten Fläche zeigen hingegen die Anteile der Archaeophyten.

Der mittlere Anteil kultivierter Arten ist am höchsten auf den Agrarflächen. Der abnehmende Verlauf mit zunehmender Flächengröße ist auch hier sehr deutlich. So sinkt der Anteil der kultivierten Arten kontinuierlich von fast $40 \%$ auf der kleinsten auf weniger als $5 \%$ auf der größten Flächengröße. Auf den Grünland- und Waldflächen kamen keine kultivierten Arten vor; auf den Siedlungsflächen liegen ihre mittleren Anteile auf fast allen Flächengrößen unter $2 \%$. Der mittlere Anteil indigener Arten ist auf den Grünland- und Waldflächen am höchsten. Auf allen Flächengrößen liegt er bei beiden Landschaftstypen bei mindestens $95 \%$. Während die mittleren Anteile der Neophyten auf Agrar- und Grünlandflächen auf allen Flächengrößen unter $5 \%$ bleiben, betragen deren Anteile auf den Siedlungsflächen immer mindestens $10 \%$. Auf den Waldflächen liegt der mittlere Anteil der Neophyten nahezu konsistent über alle Flächengrößen bei 2,6\% bis 3,3\%. Die mittleren Anteile der Archaeophyten steigen auf den Agrarflächen stetig an, auf den Siedlungsflächen sind sie auf den kleineren vier Flächengrößen variabel und auf den größeren konsistent bei ca. $6 \%$. Ähnliches gilt für die Grünlandflächen, wo sie ab der Fläche A3 einen mittleren Anteil von ca. $3 \%$ haben. Auf den Siedlungsflächen ist der mittlere Anteil der Neophyten am größten. Bei zunehmender Flächengröße sinkt der Anteil zunächst von ca. $17 \%$ auf der kleinsten auf ca. $11 \%$ auf der Fläche A4 und steigt dann stetig an auf $22 \%$ auf der größten Flächengröße. Dabei ist wie bereits in Kapitel $4.1 \mathrm{zu}$ berücksichtigen, dass der mittlere Anteil der Neophyten auf allen Flächengrößen insgesamt nur zu durchschnittlich 20,9 \% (11,6 - 27,2 \%) aus wildwachsenden Neophyten besteht (Abb. 22). Den weitaus größeren Anteil machen gepflanzte Neophyten aus, bei denen es sich auch um Zuchtformen handeln kann, die bei der Erfassung nicht von den Wildformen unterschieden wurden. 
Agrarfläche ( $n=14)$

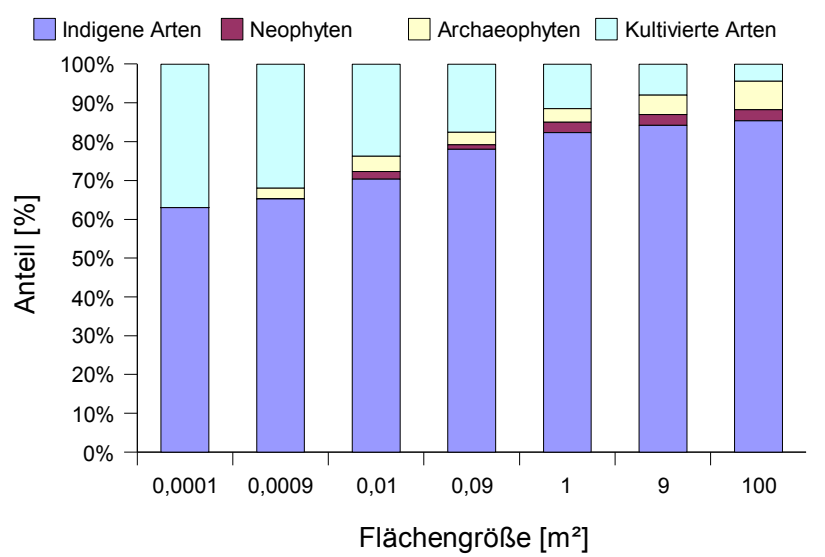

Siedlungsfläche $(n=13)$

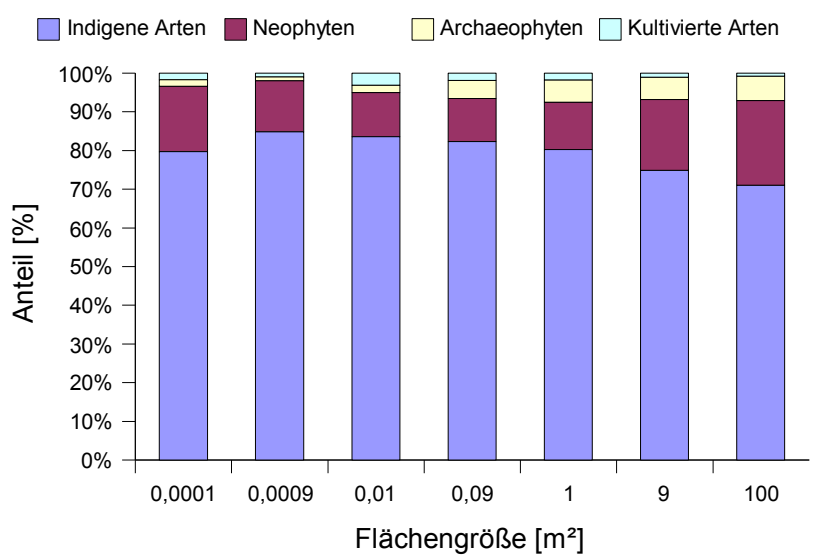

Grünland $(n=5)$

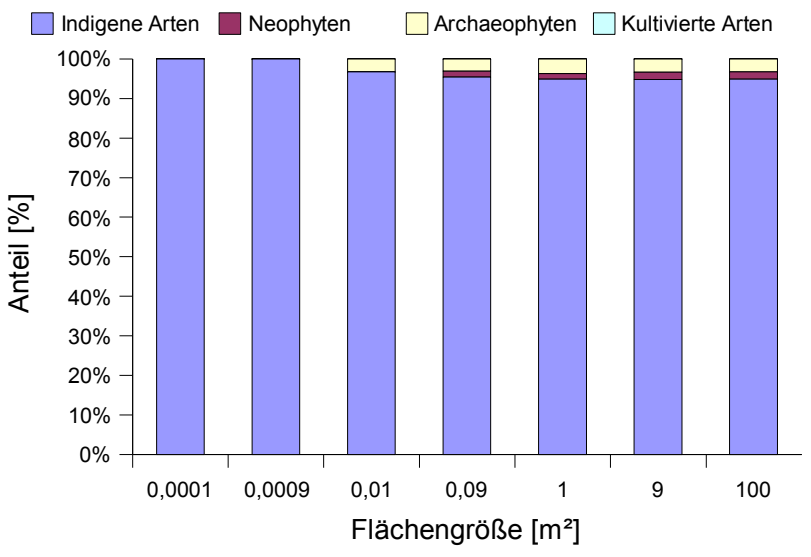

Wald $(n=18)$

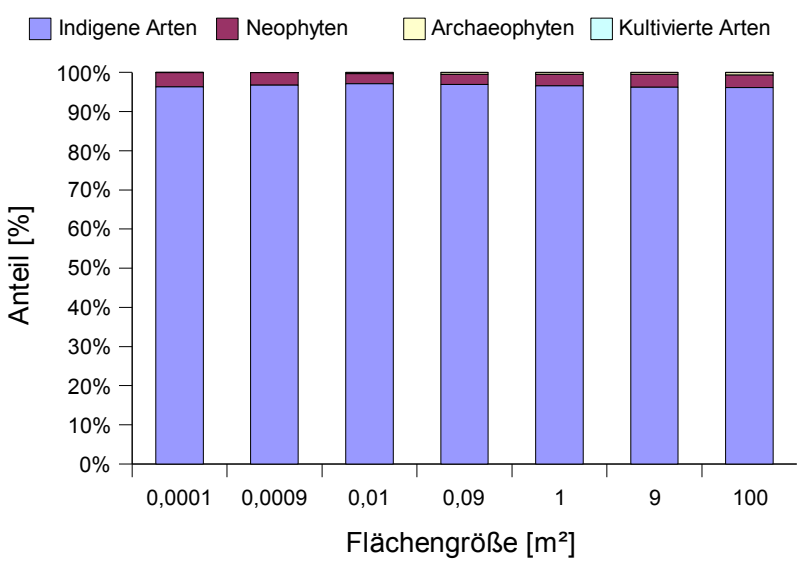

Abbildung 21: Mittlere Artenanteile der floristischen Statusgruppen differenziert nach den verschiedenen Landschaftstypen in Abhängigkeit zur Flächengröße.

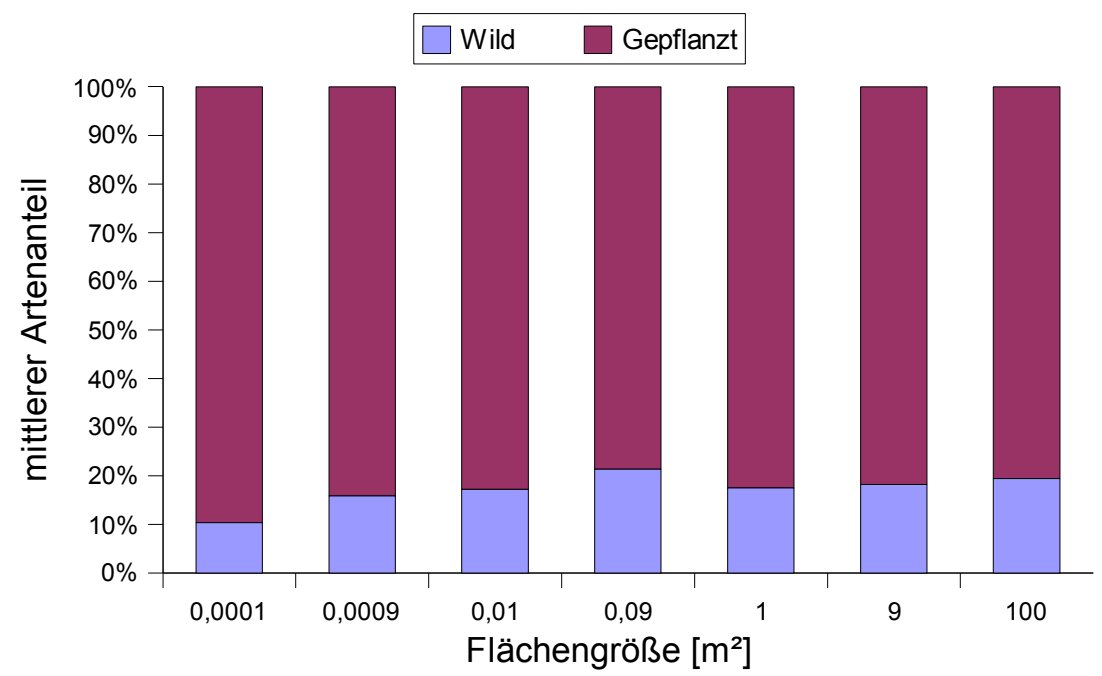

Abbildung 22: Mittlerer Artenanteil wildwachsender und gepflanzter Neophyten in Abhängigkeit zur Flächengröße im Siedlungsbereich. 


\subsubsection{Diskussion}

Die Variabilität der mittleren Anteile von Artengruppen oder floristischen Statusgruppen auf den verschiedenen Flächengrößen ist generell dadurch bedingt, dass diese Gruppen an bestimmte Substrate oder Standorte gebunden sein können. Die meisten heimischen Flechtenarten kommen epixyl, epilithisch oder epiphytisch vor und sind außerdem meist an eine dieser Schichten gebunden bei oft stenöken Standortansprüchen. Unter den Moosarten wachsen viele auch auf dem Erdboden (sogar in Pflasterritzen) und sind zusätzlich oft ubiquitär verbreitet mit euryöken Standortansprüchen. So wächst z. B. das häufige Brachythecium rutabulum. (s. Kap. 6) sowohl epiphythisch, epixyl als auch auf dem Erdboden. Das erklärt, dass die mittleren Artendichten der Moose insgesamt als auch bezogen auf die verschiedenen Landschaftstypen auf allen Flächengrößen mindestens $5 \%$ betragen, meist sogar mehr als $10 \%$. Unter den Flechten wurden nur selten einige bodenbewohnende Arten der Gattung Cladonia registriert. Ansonsten waren die Flechten stärker an das Vorkommen von Bäumen, Gestein oder Totholz gebunden. Diese Substrate tauchen mit höherer Wahrscheinlichkeit auf den größeren Flächen auf (Abb. 18). Dadurch dass im Wald hauptsächlich Bäume und Totholz als Substrat für Flechten in Frage kommen und zumindest erstere nur in gewissen Abständen zueinander stehen, sind die mittleren Anteile der Flechten innerhalb dieses Landschaftstyps auf kleineren Flächen sehr gering. Bei der Untersuchung von verschiedenen Waldtypen im Nationalpark Kuhrische Nehrung in Russland hat DoLNIK (2003a) einen vergleichbaren Trend der mittleren Artenanteile der Flechten herausgefunden. Bei allen Waldtypen steigt der Anteil der epiphytischen und epixylen Flechtenarten mit zunehmender Flächengröße. Allerdings stagniert dieser Anstieg spätestens bei einer Flächengröße von $225 \mathrm{~m}^{2}$. Während der mittlere Anteil epiphytischer und epixyler Flechtenarten zusammnen auf der kleinsten seiner berücksichtigten Flächengrößen $\left(1 \mathrm{~m}^{2}\right)$ zwischen ca. $5 \%$ und ca. $12 \%$ beträgt, liegt deren Anteil auf der Fläche A7 zusammen bei etwa $26 \%$ (ca. $22 \%$ bis ca. $34 \%$ ). Die mittleren Anteile der Flechtenarten der hier untersuchten Waldflächen ist auf beiden Flächengrößen deutlich geringer als die von DolNik (2003a) ermittelte. Der Beitrag der Flechten und Moose zur Artenvielfalt in dem hiesigen Untersuchungsgebiet ist damit geringfügiger.

Die Variation der mittleren Artenanteile hat DolNik (2006) außerdem genutzt um die minimale Flächengröße für die Untersuchung des Artenreichtums zu ermitteln. So geht er davon aus, dass Veränderungen der Artenanteile von der einen Flächengröße zur nächstgrößeren von weniger als $1 \%$ als Hinweis gedeutet werden können, „dass die jeweiligen Artengruppen für den Vegetationstyp [auf dieser größeren Flächengröße] 'repräsentativ' im Aufnahmematerial erfasst wurden“. Bei der Betrachtung der mittleren Anteile der Gesamtartenzahlen dieser Untersuchung (Abb. 17) lässt sich kein Flächenübergang ausmachen, bei dem sich die mittleren Anteile der Artengruppen zu weniger als $1 \%$ verändern. Zwar ändern sich die Artenzahlen der Gefäßpflanzen zur größten Fläche nicht stärker als $1 \%$, der Anteil der Flechten und Moose aber schon, so dass eine Annäherung an eine dauerhafte Gleichverteilung bei weiterer Flächenvergrößerung nicht zu erkennen ist. Im Vergleich zu der von DoLNIK (2003b) empfohlenen Standardflächengröße für alle Vegetationstypen von $400 \mathrm{~m}^{2}$ ist die größte Flächengröße der vorliegenden Untersuchung mit $100 \mathrm{~m}^{2}$ recht klein. Nicht vergessen werden darf dabei die Tatsache, dass Dolnik (2003a, 2003b, 2006) seine Daten in Pflanzengesellschaften erhoben hat und seine Empfehlung für den Vergleich verschiedener Gesellschaften auf ihren Artenreichtum gilt. Trotzdem ist auch im Rahmen dieser Arbeit eine möglichst repräsentative Erfassung des Artenreichtums erwünscht gewesen. Der Arbeitsaufwand, 50 geschachtelte Plots mit einer Flächengröße von $400 \mathrm{~m}^{2}$ innerhalb eines Sommers aufzunehmen, wäre für eine Person sehr groß gewesen. 
Der hohe mittlere Anteil kultivierter Arten auf den kleinen Flächengrößen ist ackerbaulich bedingt durch die systematische Anpflanzung der Arten bei möglichst hoher Individuendichte. Dadurch war bei den Plots auf den Agrarflächen die Wahrscheinlichkeit groß, dass auch auf den kleinsten Flächen Individuen der angebauten Art erfasst werden. Mit zunehmender Flächengröße spielen die kultivierten Arten bei der mittleren Gesamtartendichte keine Rolle mehr, da ich auch insgesamt nur 9 kultivierte Arten erfasst habe und diese nicht gemischt auf einer Fläche vorkommen. Dieser Effekt zeigt sich sogar innerhalb der Plots auf den Agrarflächen. Zu den wenigen kultivierten Arten kommt auf den größeren Flächengrößen eine steigende Anzahl indigener Arten, Neophyten und Archaeophyten. Den Ergebnissen dieser Arbeit zufolge machen Neophyten und Archaeophyten auf den Wald- und Grünlandflächen nur einen kleinen Teil der mittleren Artendichte aus. Die geringe Varianz im mittleren Anteil der Neophyten auf den Waldflächen liegt dabei daran, dass es sich um Neophyten der naturgemäß in Wäldern stark ausgeprägten Baum- und Strauchschicht handelt. Hierzu zählen z. B. Larix kaempferi (Japanische Lärche) und Prunus serotina (Späte Traubenkirsche). Aber auch in der Krautschicht sind einige Arten mit hoher Flächenbedeckung zu finden, wie z. B. Galeobdolon argentatum (Silberblättrige Goldnessel) und Fallopia japonica (Japanischer Staudenknöterich). Auf den Grünlandflächen finden sich hauptsächlich Conyza canadensis (Kanadisches Berufskraut) und Solidago gigantea (Riesen-Goldrute) unter den Neophyten und Plantago lanceolata (Spitz-Wegerich) bei den Archaeophyten.

Der hohe mittlere Anteil an Neophyten in den Siedlungsbereichen entspricht den Erkenntnissen von WANiA et al. (2005). Sie fanden heraus, dass der mittlere Anteil von Neophyten auf Siedlungsflächen signifikant höher ist als der mittlere Anteil auf Agrarflächen.

\subsection{Zur Variabilität der Artenzahlen}

Mit Hilfe der Standardabweichung und des Variationskoeffizienten, welcher das Verhältnis der Standardabweichung zum arithmetischen Mittel darstellt, lassen sich einige interessante Aspekte der klassischen Pflanzensoziologie beleuchten. Dazu zählt die Frage nach der notwendige Anzahl von Replikaten für repräsentative Biodiversitätsuntersuchungen (vgl. Dengler 2006). Man muss hierbei zwei Konzepte unterscheiden. Zum einen kann die Anzahl von Replikaten kleinerer Flächengrößen innerhalb eines Plots untersucht werden. Hierbei geht es darum die Artendichte mit einer bestimmten Genauigkeit zu erfassen, und das trotz steigender Variabilität der Artendichten bei abnehmender Flächengröße. Zum anderen kann man Aussagen über die notwendige Anzahl von Replikaten innerhalb eines Untersuchungsgebietes machen. Dabei steht die Erfassung der Artendichte des Untersuchungsgebietes auf einer bestimmten Flächengröße mit einer bestimmten Genauigkeit im Vordergrund. Beide Konzepte werden im Folgenden untersucht.

\subsubsection{Ergebnisse}

Für die mittleren Gesamtartenzahlen lassen sich zwei Trends feststellen. Die Standardabweichung nimmt mit zunehmender Flächengröße zu, während der Variationskoeffizient, als Maß der relativen Streuung, mit zunehmender Flächengröße zunächst abnimmt und dann wieder steigt (Tab. 11). So liegt die Standardabweichung auf Fläche A1 bei 1,0 und auf der Fläche A7 bei 25,5. Der 
Variationskoeffizient auf der kleinsten Fläche liegt bei 63,2 \%, auf der Fläche A4 bei 54,0 \% und auf der größten Fläche bei 65,9\%. Die Standardabweichung lässt sich in Abhängigkeit zur logarithmierten Flächengröße $(\log 10)$ hervorragend durch eine Exponentialfunktion approximieren (Abb. 23 A). Dieser Zusammenhang ist hoch signifikant mit $p<0,001$. Die Anpassung wird dabei maßgeblich durch den Wert der Fläche A7 gestört. Unter Ausschluss dieses Wertes zeigt die Kurvenanpassung einen besseren Verlauf mit einem höheren Anteil erklärter Varianz (Abb. 23 B).

A

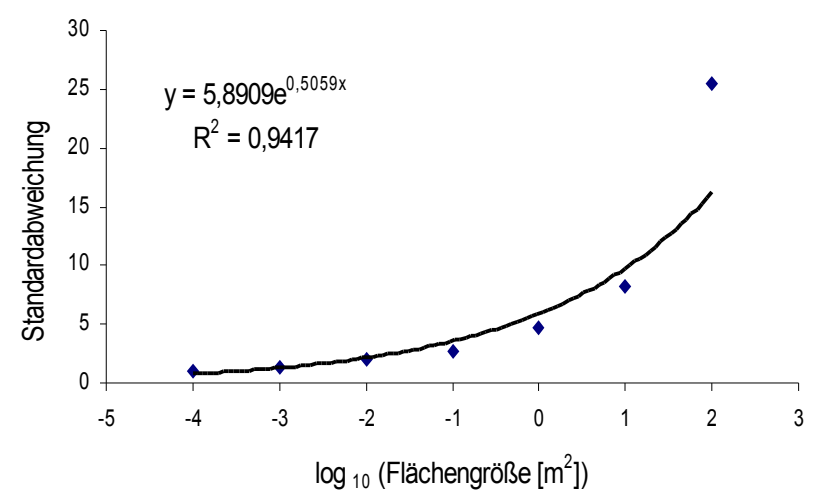

B

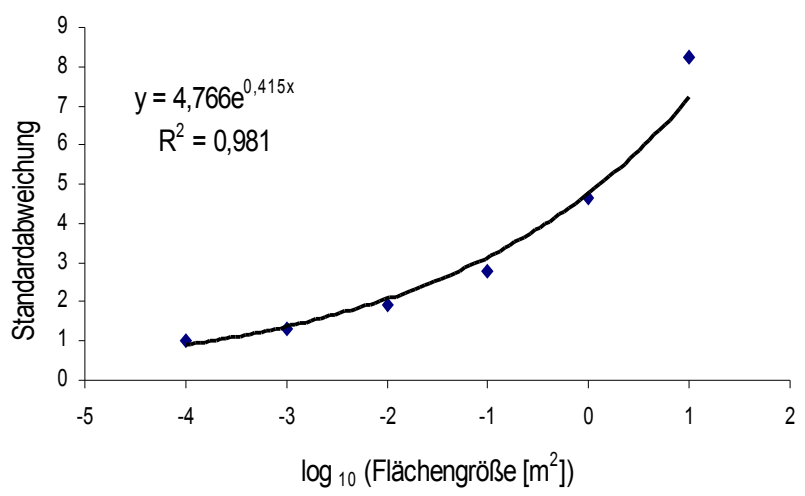

Abbildung 23: Mittlere Standardabweichung der Artenzahl auf unterschiedlich großen Flächen bei logarithmierter Flächengröße unter Berücksichtigung aller Flächengrößen (A) und unter Ausschluss der Fläche A7 (B).

Der Variationskoeffizient in Abhängigkeit zur logarithmierten Flächengröße lässt sich mit keiner Funktion mit nur zwei Funktionsparametern vernünftig approximieren, da er mit zunehmender Flächengröße zunächst fällt und dann wieder steigt. Dieser Verlauf lässt sich am besten mit einer polynomischen Funktion zweiten Grades wiedergeben (s. Kap. 3.4.1). Das Signifikanzniveau bleibt hier aber geringer mit $\mathrm{p}<0,05$.

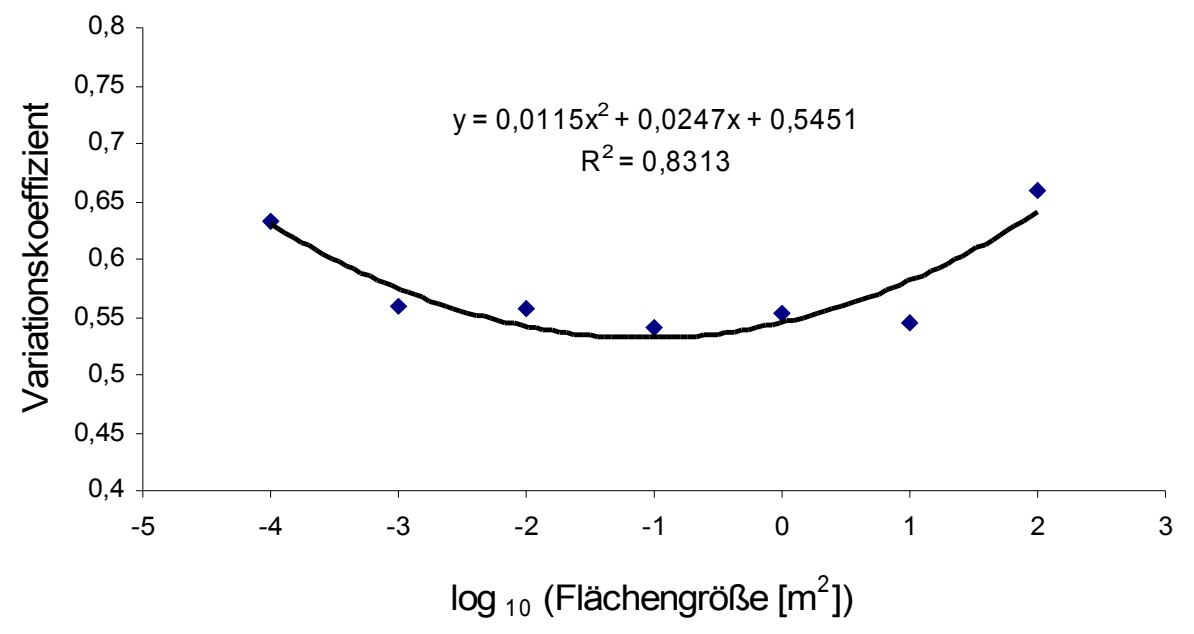

Abbildung 24: Mittlerer Variationskoeffizient der Artenzahl in Abhängigkeit zur logarithmierten Flächengröße.

Bei einer Differenzierung der verschiedenen Landschaftstypen zeigen sich die gleichen Ergebnisse. 
Die Standardabweichung kann in Abhängigkeit zur Fläche auch differenziert nach Artengruppen oder Landschaftstypen signifikant und mit einem hohen Anteil erklärter Varianz mit einer Exponentialfunktion approximiert werden (Tab 9).

Tabelle 9: Mittleres Bestimmtheitsmaß und Signifikanzniveau der Exponentialfunktion der Standardabweichung der Artendichten insgesamt auf den verschiedenen Landschaftstypen sowie der Artengruppen.

\begin{tabular}{lrr}
\hline & $R^{2}$ & $p$ \\
\hline Agrarfläche $(\mathrm{n}=14)$ & 0,981 & $<0,001$ \\
Grünland (n=5) & 0,994 & $<0,001$ \\
Siedlungs fläche $(\mathrm{n}=13)$ & 0,987 & $<0,001$ \\
Wald $(\mathrm{n}=18)$ & 0,961 & $<0,001$ \\
Gefäßpflanzen & 0,981 & $<0,050$ \\
Moose & 0,949 & $<0,001$ \\
Flechten & 0,995 & $<0,001$ \\
\hline
\end{tabular}

Die Werte des Variationskoeffizienten streuen in allen Fällen sehr stark, so dass in kaum einem Fall mit einer Exponentialfunktion ein hoher Anteil erklärter Varianz erreicht wird (Tab 10). Das Signifikanzniveau von c, also der Steigung der Funktion, ist zum Teil trotzdem sehr hoch.

Tabelle 10: Mittleres Bestimmtheitsmaß und Signifikanzniveau der Exponentialfunktion und der polynomischen Funktion des mittleren Variationskoeffizienten der verschiedenen Landschaftstypen sowie der Artengruppen mit den Werten der Funktionsparameter der Exponentialfunktion.

\begin{tabular}{lrrrr}
\hline & \multicolumn{4}{c}{ Exponentialfunktion } \\
& $R^{2}$ & $p$ & $a$ & $b$ \\
\hline Agrarfläche $(\mathrm{n}=14)$ & 0,529 & $>0,050$ & 0,782 & $-0,034$ \\
Grünland $(\mathrm{n}=5)$ & 0,013 & $<0,001$ & 0,551 & $-0,006$ \\
Siedlungs fläche $(\mathrm{n}=13)$ & 0,697 & $<0,001$ & 0,470 & $-0,096$ \\
Wald (n=18) & 0,023 & $<0,001$ & 0,363 & $-0,008$ \\
Gefäßpflanzen & 0,753 & $>0,050$ & 0,613 & $-0,028$ \\
Moose & 0,944 & $<0,001$ & 0,891 & $-0,146$ \\
Flechten & 0,836 & $=0,001$ & 1,849 & $-0,221$ \\
\hline
\end{tabular}

\begin{tabular}{|c|c|c|c|c|c|}
\hline & \multicolumn{5}{|c|}{ Polynomische Funktion } \\
\hline & $R^{2}$ & $p$ & $a$ & $b$ & $c$ \\
\hline Agrarfläche $(\mathrm{n}=14)$ & 0,697 & $>0,050$ & 0,009 & $-0,043$ & 0,76 \\
\hline Grünland $(\mathrm{n}=5)$ & 0,014 & $>0,050$ & 0,000 & $-0,004$ & 0,55 \\
\hline Siedlungs fläche $(\mathrm{n}=13)$ & 0,977 & $<0,050$ & 0,020 & $-0,006$ & 0,42 \\
\hline Wald $(n=18)$ & 0,381 & $>0,050$ & 0,007 & $-0,010$ & 0,38 \\
\hline Gefäßpflanzen & 0,426 & $<0,001$ & 0,008 & $-0,033$ & 0,59 \\
\hline Moose & 0,995 & $<0,001$ & 0,031 & $-0,084$ & 0,84 \\
\hline Flechten & 0,829 & $>0,050$ & 0,039 & $-0,465$ & 1,88 \\
\hline
\end{tabular}


Zur Überprüfung der Methode der zufällig verteilten Plots und der gewählten Anzahl von Plots, sowie zur Ermittlung der notwendigen Anzahl von Replikaten einer bestimmten Flächengröße, um die mittlere Artendichte mit einer bestimmten Genauigkeit zu erfassen, kann die mathematische Beziehung zwischen relativem Fehler und Variationskoeffizient genutzt werden (vgl. LozÁN \& KaUSCH 2004):

$$
S E_{\text {relativ }}=\frac{V}{\sqrt{n}}
$$

Für diese Auswertung wurde von jedem Plot nur eine Artendichte je Teilfläche berücksichtigt. Alle vier Teilflächen der kleineren Flächen zu benutzen, wäre unzulässig, da die Positionen der vier Teilflächen immer durch die systematische Anordnung der Teilflächen innerhalb eines Plots vorgegeben ist. Genau wie der gesamte Plot, kann deshalb nur eine der Teilflächen als zufällig verteilt innerhalb des Untersuchungsgebiets betrachtet werden. Es zeigt sich, dass mit der Wahl von 50 Plots die mittlere Gesamtartendichte mit einer Genauigkeit von durchschnittlich 90,3\% ermittelt wurde (Tab 11). Wollte man den relativen Standardfehler halbieren, müsste man die Anzahl der Replikate vervierfachen.

Tabelle 11: Standardabweichung (SD) und Variationskoeffizient (V) von 50 Stichproben je Flächengröße, die notwendige Anzahl von Replikaten bei einer bestimmten Genauigkeit bei der Erfassung der Artendichte sowie die erzielte Genauigkeit mit 50 Stichproben; SE = relativer Standardfehler.

\begin{tabular}{|c|c|c|c|c|c|c|}
\hline \multirow[b]{2}{*}{ Fläche $\left[\mathrm{m}^{2}\right]$} & \multirow[b]{2}{*}{$S D$} & \multicolumn{4}{|c|}{ Anzahl Replikate bei: } & \multirow{2}{*}{$\begin{array}{l}\text { SE bei } \\
N=50\end{array}$} \\
\hline & & $V K$ & $S E<10 \%$ & $S E<5 \%$ & SE wie $9 \mathrm{~m}^{2}$ & \\
\hline 0,0001 & 1,28 & 0,79 & 62,04 & 248,16 & 76 & $11,1 \%$ \\
\hline 0,0009 & 1,82 & 0,73 & 53,91 & 215,66 & 66 & $10,4 \%$ \\
\hline 0,01 & 2,43 & 0,68 & 46,21 & 184,83 & 56 & $9,6 \%$ \\
\hline 0,09 & 3,25 & 0,65 & 42,03 & 168,12 & 51 & $9,2 \%$ \\
\hline 1 & 5,85 & 0,67 & 44,55 & 178,20 & 54 & $9,4 \%$ \\
\hline 9 & 10,40 & 0,64 & 40,99 & 163,96 & 50 & $9,1 \%$ \\
\hline 100 & 25,51 & 0,66 & 43,46 & 173,85 & 53 & $9,3 \%$ \\
\hline
\end{tabular}

\subsubsection{Diskussion}

Sowohl den Anstieg der Standardabweichung als auch die Abnahme des Variationskoeffizienten bei zunehmender Flächengröße hat DeNGLER (2006) in Trockenrasenverbänden untersucht. In allen Fällen sind die exponentiellen Zusammenhänge signifikant, weshalb er die beiden Zusammenhänge als Gesetzmäßigkeit postuliert unter der Annahme, dass sie auch für andere Vegetationstypen gelten. Im Rahmen dieser Untersuchung lässt sich der Anstieg der Standardabweichung der mittleren Artenzahlen insgesamt als auch differenziert nach den verschiedenen Landschaftstypen und den untersuchten Artengruppen ebenfalls signifikant $(\mathrm{p}<0,001)$ mit einer Exponentialfunktion wiedergeben. Für den Variationskoeffizienten trifft dies jedoch lediglich für die Artenzahlen der Plots auf den Siedlungsflächen zu, sowie für die jeweils gesondert betrachteten Flechten und Moose. Insgesamt streuen die Werte des Variationskoeffizienten viel stärker als die der 
Standardabweichung. Das liegt an der zufälligen Verteilung der Aufnahmeflächen im Untersuchungsgebiet. Dengler (2006) kam deshalb zu signifikanten Ergebnissen bei der Anpassung mit einer Exponentialfunktion, weil er bei seinen Untersuchungen nur in sich homogene Trockenrasenverbände berücksichtigt hat.

Da weder die polynomische Funktion zweiten Grades noch die Exponentialfunktion die Beziehung des Variationskoeffizienten zur Flächengröße gut wiedergeben, können sie nicht herangezogen werden um generelle Vorschläge für die notwendige Anzahl von Replikaten bestimmter Flächengrößen innerhalb eines Plots abzuleiten.

Trotzdem konnte mit Hilfe des Variationskoeffizienten und seiner mathematischen Beziehung zum relativen Standardfehler eine Aussage über die Höhe des für diese Arbeit gewählten Stichprobenumfangs getroffen werden. In einer strukturell heterogenen mitteleuropäischen Landschaft kann man die mittlere Artendichte in einem Untersuchungsgebiet von $126 \mathrm{~km}^{2}$ Größe mit 50 zufälligen Stichproben mit einer Genauigkeit von durchschnittlich $90 \%$ ermitteln. Dabei reicht die Spanne von 41 notwendigen Replikaten bei der Fläche A6 bis 62 notwendigen Replikaten bei der Fläche A1. Für dieses Untersuchungsgebiet hätte man also, um die Artendichten auf allen Flächengrößen mit mindestens $90 \%$ Genauigkeit $\mathrm{zu}$ erfassen, für die beiden kleinsten Flächengrößen noch zwölf bzw. vier weitere Aufnahmen dieser Flächengrößen machen müssen. Für eine gleichbleibende Genauigkeit entsprechend der Flächengröße auf der die Artendichte am genauesten erfasst wurde, hätte man die Replikate den Angaben in Tabelle 11 entsprechend erhöhen müssen.

Insgesamt ist ein relativer Standardfehler von $10 \%$ recht hoch, allerdings ist bei der Wahl der Anzahl von Replikaten natürlich immer die Bearbeitungszeit zu berücksichtigen. Aus dieser Perspektive betrachtet, ist die Verdopplung des relativen Standardfehlers eine vertretbare Reduzierung der Genauigkeit, wenn man dabei die Anzahl notwendiger Replikate um ein Viertel reduzieren kann. 


\section{Arten-Areal-Beziehungen}

In diesem Kapitel wird es um zwei fundamentale Aspekte der Artenzahl-Areal-Beziehungen gehen. Dies sind zum einen Artenzahl-Areal-Kurven und die Anpassungsgüte verschiedener mathematischer Funktionen, die diese Beziehung beschreiben und zum anderen der z-Wert, welcher die Steigung dieser Beziehung wiedergibt.

\subsection{Welche Funktion beschreibt den Zusammenhang von Artenzahl und Arealgröße am besten?}

Es werden die unter Kap. 3.4.2 aufgeführten Funktionen anhand der ebenfalls in diesem Kapitel beschriebenen Gütekriterien auf ihre Güte zur Wiedergabe der Artenzahl-Areal-Beziehung getestet.

\subsubsection{Ergebnisse}

Betrachtet man zunächst alle 350 Artenzahl-Areal-Wertepaare dieser Studie, zeigt sich, dass die Artendichten grundsätzlich mit zunehmender Flächengröße ansteigen. Nur in wenigen Fällen bleiben die Artendichten über mehrere Flächengrößen konstant (Abb. 25).

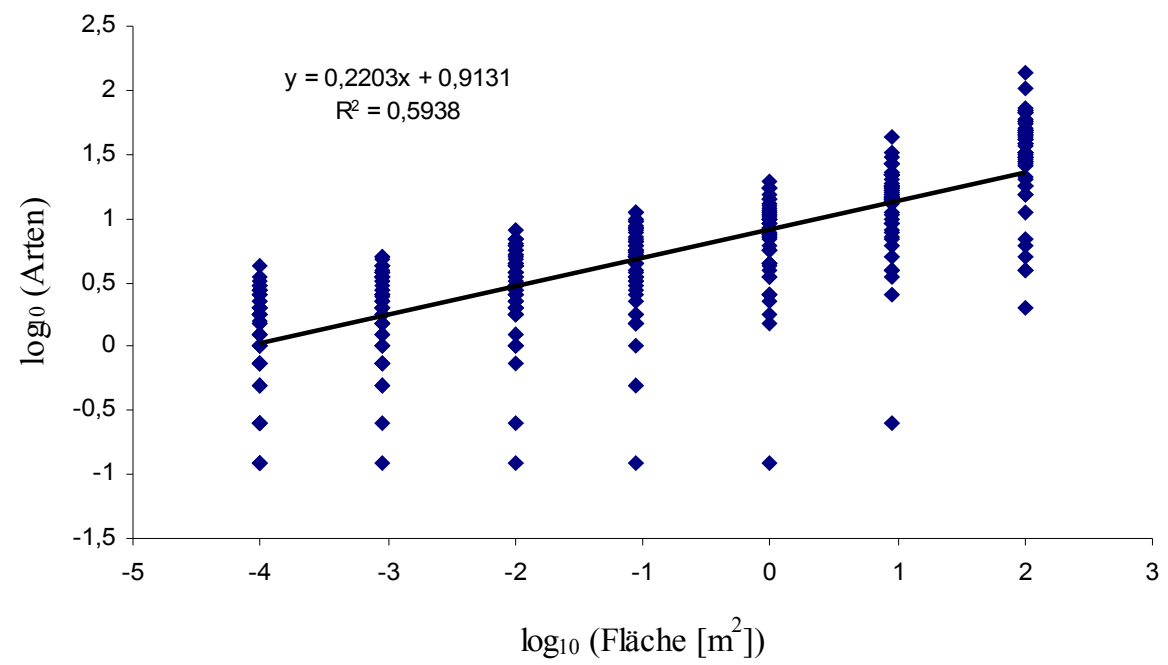

Abbildung 25: Doppelt logarithmische Darstellung der Artenzahl-ArealgrößeWertepaare aller Plots.

Werden die Arten-Areal-Kurven für alle 50 Plots mit den verschiedenen Funktionen approximiert und die Gütekriterien berechnet, können die Werte der vier Gütekriterien zunächst über die 50 Plots gemittelt werden (Tab. 12), wobei aber zu beachten ist, dass sich das Bestimmtheitsmaß einer im logarithmierten Raum gefitteten Kurve nicht mit dem einer im nicht-logarithmierten Raum gefitteten Kurve vergleichen lässt.

Der relative Extrapolationsfehler nimmt lediglich bei den beiden Formen der Potenzfunktion sowohl positive als auch negative Werte an. Während er bei den linearen Funktionen immer positiv 
ist, ist er bei den Logarithmusfunktionen und der Michaelis-Menten-Funktion immer negativ.

Sowohl für die im logarithmierten als auch im nicht-logarithmierten Raum angepassten Kurven ist das Bestimmtheitsmaß der Potenzfunktion über alle Plots gemittelt am höchsten. Das durchschnittliche Bestimmtheitsmaß der Logarithmus-Funktion liegt im nicht-logarithmierten Raum unter dem der linearen Funktion. Im logarithmierten Raum ist dies umgekehrt. Das Bestimmtheitsmaß der linearisierten Form der Michaelis-Menten-Funktion ist nicht mit den anderen Werten vergleichbar. Die Werte stellen Artefakte der Kehrwertwertbildung dar, bei der die Artenzahlen inkonsistent transformiert werden, nämlich durch die Division mit der Flächengröße.

Tabelle 12: Bestimmtheitsmaß $\left(\mathrm{R}^{2}\right)$, relativer Extrapolationsfehler (rel. EE), mittlerer relativer Residuenfehler ( $\varnothing$ rel. RE) und maximaler relativer Residuenfehler (max. rel. RE) der getesteten Funktionen über alle Plots $(\mathrm{n}=50)$ gemittelt mit Standardabweichung.

\begin{tabular}{lrrrr}
\hline Funktion & $R^{2}$ & rel. EE & Ørel. RE & Max. rel. RE \\
\hline Linear(S) & $0,88 \pm 0,10$ & $283 \pm 114 \%$ & $96 \pm 102 \%$ & $358 \pm 551 \%$ \\
Linear(log S) & $0,70 \pm 0,13$ & $435 \pm 173 \%$ & $55 \pm 27 \%$ & $148 \pm 117 \%$ \\
Potenz(S) & $0,98 \pm 0,01$ & $31 \pm 20 \%$ & $31 \pm 12 \%$ & $72 \pm 37 \%$ \\
Potenz(log S) & $0,94 \pm 0,07$ & $41 \pm 18 \%$ & $24 \pm 18 \%$ & $58 \pm 125 \%$ \\
Logarithmus (S) & $0,72 \pm 0,1$ & $56 \pm 15 \%$ & $149 \pm 165 \%$ & $556 \pm 832 \%$ \\
Logarithmus (log S) & $0,77 \pm 0,11$ & $66 \pm 17 \%$ & $45 \pm 29 \%$ & $94 \pm 80 \%$ \\
Michaelis -Menten & $0,98 \pm 0,04$ & $54 \pm 16 \%$ & $69 \pm 8 \%$ & $111 \pm 33 \%$ \\
\hline
\end{tabular}

Wird ein Ranking des Bestimmtheitsmaßes der Kurven durchgeführt, die jeweils im gleichen Raum gefittet werden, kann das durchschnittlich höhere Bestimmtheitsmaß der Potenzfunktion in beiden Fällen bestätigt werden (Tab. 13). Die Standardabweichung beider Potenzfunktionen ist dabei sehr niedrig. Auch die Intensität des Unterschiedes des Bestimmtheitsmaßes der Logarithmusfunktion und der linearen Funktion kann mit dem Ranking unterstrichen werden. Während die lineare Funktion im nicht-logarithmierten Raum einen deutlich besseren mittleren Rang einnimmt als die Logarithmusfunktion im selben Raum, ist die durchschnittlich bessere Position der Logarithmusfunktion im logarithmierten Raum nicht sehr stark. Die im Vergleich zum nichtlogarithmierten Raum höheren Werte der Standardabweichung bestätigen dies.

Tabelle 13: Ranking des Bestimmtheitsmaßes mit Mittelwert (Ø) und Standardabweichung (SD) getrennt nach dem Raum, in dem die Kurven approximiert wurden.

\begin{tabular}{lrrrrrr}
\hline Funktion & $\mathbf{1}$ & $\mathbf{2}$ & $\mathbf{3}$ & $\mathbf{4}$ & $\boldsymbol{\emptyset}$ & SD \\
\hline Linear (S) & 1 & 28 & 15 & 6 & 2,52 & 0,74 \\
Potenz (S) & 47 & 3 & 0 & 0 & 1,06 & 0,24 \\
Logarithmus (S) & 1 & 5 & 3 & 41 & 3,68 & 0,74 \\
Michaelis-Menten (S) & 1 & 14 & 32 & 3 & 2,74 & 0,6 \\
\hline Linear (log S) & 4 & 14 & 32 & - & 2,56 & 0,64 \\
Potenz (log S) & 44 & 6 & 0 & - & 1,12 & 0,33 \\
Logarithmus (log S) & 2 & 30 & 18 & - & 2,32 & 0,55 \\
\hline
\end{tabular}


Die Häufigkeiten der Ränge der übrigen unter 3.4.2 vorgestellten Gütekriterien können unter allen sieben Kurven verglichen werden, da sich diese auf Werte beziehen, die mit den ermittelten Funktionsparametern berechnet werden. Außerdem können diese Werte in allen Fällen an den nicht-logarithmierten Artendichten gemessen werden, da sie nur für diese berechnet wurden. Das bedeutet, dass die Funktionsparameter der Funktionen, die im logarithmierten Raum approximiert wurden (also mit logarithmierter Artendichte und logarithmierter Flächengröße) genutzt werden können, um nicht-logarithmische Schätzwerte für die Artendichte zu berechnen. Dazu nehme ich als Prädiktor- Variable die nicht-logarithmierte Flächengröße. Sowohl der mittlere und relative Fehler der Residuen als auch der relative Extrapolationsfehler beziehen sich auf Abweichungen dieser im nicht-logarithmierten Raum entstandenen Schätzwerte.

Anhand von ANOVAs, durchgeführt für die vier Gütekriterien, zeigt in allen vier Fällen, dass die Werte der sieben Funktion nicht immer signifikant voneinander unterschiedlich sind.

Es zeigt sich bei diesen Kriterien in den meisten Fällen eine weite Streuung über die Funktionen (Tab. 14). Trotzdem scheint es, als würden einige Funktionen ein bestimmtes Kriterium in einem überdurchschnittlichen Ausmaß mit einem bestimmten Rang belegen. Die Frequenz des Ranges dieses Kriteriums bei dieser Funktion ist dementsprechend hoch (Tab. 14, fett gedruckte Angaben). Besonders prägnantes Beispiel ist der Extrapolationsfehler, bei dem die beiden linearen Funktionen jeweils 48 mal einen bestimmten Rang eingenommen haben. Die im nicht-logarithmierten Raum approximierte lineare Funktion nimmt demnach zu 96\% den sechsten Rang ein, während die gleiche Funktion im logarithmierten Raum gefittet, ebenfalls zu 96 \%, auf dem siebten Rang liegt. Während die nicht-logarithmierte Variante der Funktion auch beim mittleren und maximalen relativen Fehler die höchste Frequenz beim Rang sechs hat, liegen die höchsten Frequenzen der logarithmierten Form bei Rang vier (mittlerer Fehler) bzw. bei Rang fünf (maximaler Fehler). Insgesamt streuen die Ranghäufigkeiten bei der logarithmierten linearen Funktion mehr. Die Funktion hat die beiden Kriterien für jeweils drei Plots, am besten erfüllt.

Die beiden Formen der Potenzfunktion haben die höchsten Frequenzen bei den niedrigen Rängen. Dabei vereint die Potenzfunktion in nicht-logarithmierter Form die höchsten Frequenzen des ersten Ranges beim relativen Extrapolationsfehler auf sich, während die logarithmierte Form beim mittleren und maximalen relativen Fehler der Residuen die Mehrheit der ersten Ränge besitzt und zwar zu $78 \%$ und $80 \%$. Beim relativen Extrapolationsfehler liegt die logarithmierte Form mit $66 \%$ auf Platz zwei, was die nicht-logarithmierte Form in keinem der anderen beiden Fällen schafft. Keines der Gütekriterien wird von der Logarithmusfunktion bei nicht-logarithmierter Artendichte mehrheitlich auf einem der ersten drei Ränge erfüllt. Für den mittleren und maximalen relativen Fehler der Residuen hat diese Form die höchsten Frequenzen aller Funktionen bei Rang sieben. Lediglich bei der Extrapolation liegt die Mehrheit der Werte auf den Rängen drei und vier. Die logarithmierte Form schneidet bei diesem Kriterium schlechter ab als die nicht-logarithmierte, bei den beiden übrigen Kriterien besser.

Die Standardabweichung der Michaelis-Menten-Funktion gemittelt über die drei Kriterien ist relativ hoch. Die höchsten Rangfrequenzen befinden sich im mittleren Bereich. 
Tabelle 14: Frequenz der Rangpositionen der getesten Funktionen im logarithmierten und nichtlogarithmierten Raum für die Gütekriterien mittlerer relativer Fehler ( rel. E), maximaler relativer Fehler (max. rel. E) und relativer Extrapolationsfehler (rel. EE), sowie deren Mittelwert (Ø) und Standardabweichung (SD). Die maximal erreichten Frequenzen jedes Kriteriums und jeden Ranges sind fett gedruckt.

\begin{tabular}{|c|c|c|c|c|c|c|c|c|c|c|c|c|c|c|c|c|c|c|}
\hline \multirow[b]{2}{*}{ Rang } & \multicolumn{9}{|c|}{ Funktionen im nicht-logarithmierten Raum } & \multicolumn{9}{|c|}{ Funktionen im logarithmierten Raum } \\
\hline & 1 & 2 & 3 & 4 & 5 & 6 & 7 & $\varnothing$ & SD & 1 & 2 & 3 & 4 & 5 & 6 & 7 & $\varnothing$ & SD \\
\hline & \multicolumn{9}{|c|}{ Linear (S) } & \multicolumn{9}{|c|}{ Linear $(\log S)$} \\
\hline Ø rel. E & 0 & 2 & 1 & 2 & 17 & 23 & 5 & 5,46 & 1,07 & 3 & 3 & 5 & 28 & 6 & 4 & 1 & 3,94 & 1,24 \\
\hline Max. rel. E & 0 & 2 & 0 & 0 & 4 & 34 & 12 & 6,24 & 0,97 & 3 & 4 & 2 & 16 & 22 & 3 & 0 & 4,18 & 1,26 \\
\hline rel. EE & 1 & 0 & 0 & 0 & 0 & 48 & 1 & 5,92 & 0,72 & 0 & 1 & 0 & 0 & 0 & 1 & 48 & 6,88 & 0,72 \\
\hline & \multicolumn{9}{|c|}{ Potenz (S) } & \multicolumn{9}{|c|}{ Potenz $(\log S)$} \\
\hline$\varnothing$ rel. E & 6 & 29 & 10 & 4 & 1 & 0 & 0 & 2,30 & 0,86 & 39 & 6 & 4 & 0 & 1 & 0 & 0 & 1,36 & 0,80 \\
\hline Max. rel. E & 4 & 19 & 23 & 4 & 0 & 0 & 0 & 2,54 & 0,76 & 40 & 5 & 2 & 0 & 2 & 1 & 0 & 1,44 & 1,11 \\
\hline rel. EE & 37 & 8 & 2 & 2 & 1 & 0 & 0 & 1,44 & 0,91 & 9 & 33 & 4 & 1 & 2 & 1 & 0 & 2,14 & 1,01 \\
\hline & \multicolumn{9}{|c|}{ Logarithmus (S) } & \multicolumn{9}{|c|}{ Logarithmus $(\log S)$} \\
\hline Ø rel. E & 0 & 1 & 0 & 4 & 3 & 7 & 35 & 6,40 & 1,12 & 2 & 8 & 29 & 6 & 4 & 0 & 1 & 3,12 & 1,04 \\
\hline Max. rel. E & 0 & 0 & 1 & 0 & 3 & 7 & 39 & 6,66 & 0,77 & 2 & 19 & 18 & 7 & 3 & 0 & 1 & 2,88 & 1,12 \\
\hline rel. EE & 0 & 2 & 18 & 28 & 2 & 0 & 0 & 3,60 & 0,64 & 1 & 0 & 1 & 3 & 44 & 0 & 1 & 4,86 & 0,73 \\
\hline \multicolumn{10}{|c|}{ Michaelis -Menten } & & & & & & & & & \\
\hline Ø rel. E & 0 & 1 & 1 & 6 & 18 & 16 & 8 & 5,42 & 1,09 & & & & & & & & & \\
\hline Max. rel. E & 1 & 1 & 4 & 23 & 16 & 5 & 0 & 4,34 & 0,98 & & & & & & & & & \\
\hline rel. EE & 2 & 6 & 25 & 16 & 1 & 0 & 0 & 3,16 & 0,82 & & & & & & & & & \\
\hline
\end{tabular}

Mittelt man die mittleren Ränge dieser drei Kriterien über alle Funktionen, ist der Potenzfunktion im logarithmierten Raum die höchste Güte bei der Beschreibung der Artenzahl-Areal-Beziehungen zuzuschreiben (Tab. 15).

Die durchschnittlichen Ränge der Funktionen sind beim mittleren und maximalen relativen Residuenfehler meist sehr ähnlich. Diesen Zusammenhang bestätigt auch eine Korrelationsanalyse der Gütekriterien. Demnach korrelieren mittlerer und maximaler relativer Residuenfehler signifikant mit $r=0,94$. Deshalb können beide Werte zuvor unter sich gemittelt werden und zu einem relativen Residuenfehler zusammengeführt werden. Ein mittleres Ranking der auf diese Weise reduzierten Kriterien befürwortet die Potenzfunktion im nicht-logarithmierten Raum als bestgeeignete Funktion für die Beschreibung der Artenzahl-Areal-Beziehungen.

Tabelle 15: Mittleres Ranking der Funktionen für alle Kriterien mit Standardabweichung, sowie der Mittelwert über alle Kriterien.

\begin{tabular}{lrrrrr}
\hline Funktion & $\mathbf{R}^{\mathbf{2}}$ & $\boldsymbol{\varnothing}$ rel. E & Max. rel. E & rel. EE & Mittelwert \\
\hline Potenz (S) & $1,04 \pm 0,20$ & $2,30 \pm 0,86$ & $2,54 \pm 0,76$ & $1,44 \pm 0,91$ & 1,83 \\
Linear (S) & $2,12 \pm 0,39$ & $5,46 \pm 1,07$ & $5,96 \pm 0,97$ & $5,92 \pm 0,72$ & 4,87 \\
Logarithmus (S) & $2,84 \pm 0,42$ & $6,40 \pm 1,12$ & $6,66 \pm 0,77$ & $3,60 \pm 0,64$ & 4,88 \\
\hline Potenz (log S) & $1,06 \pm 0,24$ & $1,36 \pm 0,80$ & $1,44 \pm 1,11$ & $2,14 \pm 1,01$ & 1,5 \\
Logarithmus (log S) & $3,68 \pm 0,74$ & $3,12 \pm 1,04$ & $2,88 \pm 1,12$ & $4,86 \pm 0,73$ & 3,64 \\
Linear (log S) & $2,52 \pm 0,74$ & $3,94 \pm 1,24$ & $4,18 \pm 1,26$ & $6,88 \pm 0,72$ & 4,38 \\
\hline Michaelis-Menten (A/S) & & $5,42 \pm 1,09$ & $4,34 \pm 0,98$ & $3,16 \pm 0,82$ & 4,31 \\
\hline
\end{tabular}


Da man das Bestimmtheitsmaß nur innerhalb des gleichen Raumes miteinander vergleichen kann, werden die Kurven der Potenzfunktion in ihrer nicht-logarithmierten Form jeweils für logarithmierte Artenzahlen und umgekehrt approximiert. Ich beschränke mich dabei auf die beiden Formen der Potenzfunktion, da diese bei allen Kriterien durchschnittlich am besten abgeschnitten haben. Zwar ist das Bestimmtheitsmaß der Potenzfunktion im logarithmierten Raum gefittet für nicht-logarithmierte Artenzahlen höher $\left(\mathrm{R}^{2}=0,873\right)$ als dies umgekehrt der Fall ist $\left(\mathrm{R}^{2}=0,763\right)$, jedoch deutlich schlechter als das Bestimmtheitsmaß derselben Funktion im nicht-logarithmierten Raum gefittet für nicht-logarithmierte Artenzahlen $\left(\mathrm{R}^{2}=0,982\right)$.

Demnach stellt sie den Kriterien entsprechend die beste Funktion für die Approximation der Artendichte in Abhängigkeit zur Flächengröße dar. An zweiter Stelle kommt die logarithmierte Form der Potenzfunktion, die den Kriterien insgesamt fast genauso gut entsprach wie die nichtlogarithmierte Form. Die Logarithmusfunktion mit logarithmierten Artendichten kommt zu einem besseren Ergebnis als bei nicht-logarithmierten Artendichten, liegt aber mit dem mittleren Ranking noch deutlich hinter der Michaelis-Menten-Funktion, die aufgrund ihrer hohen Positionierung beim Bestimmtheitsmaß (Rang zwei) auf den dritten Rang kommt. Die beiden Formen der linearen Funktion liegen dicht beieinander auf den Rängen fünf und sechs.

Abbildung 26 zeigt die Verläufe der Regressionskurven aller berücksichtigter Funktionen für einen Plot auf einer Siedlungsfläche.

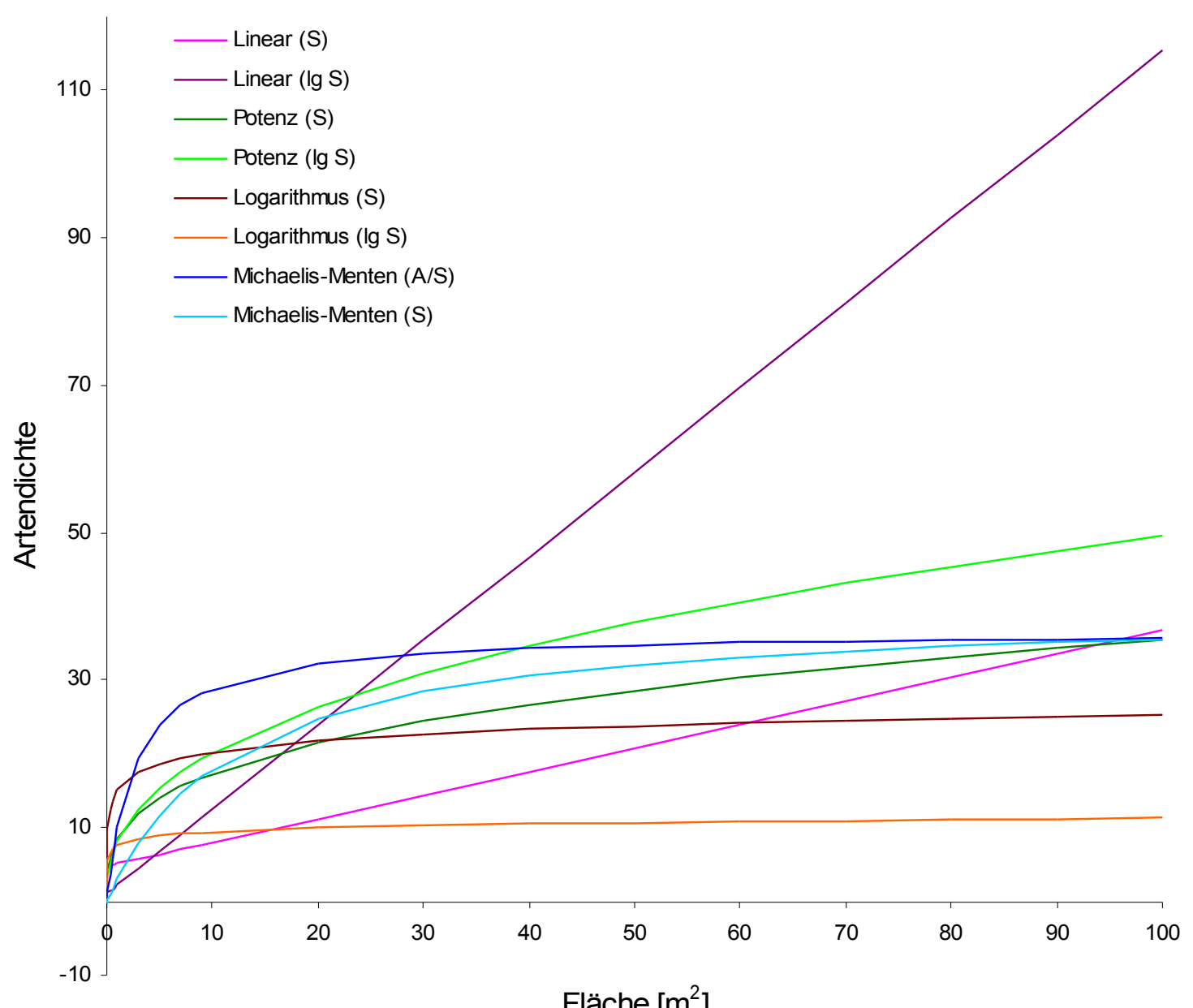

Abbildung 26: Kurvenverlauf der getesteten Funktionen exemplarisch dargestellt anhand eines Plots. 
Korrelationen des Bestimmtheitsmaßes und des relativen Extrapolationsfehlers über alle Funktionen zeigen, dass sowohl die Potenzfunktionen beider Formen als auch die beiden Logarithmusfunktionen signifikant miteinander korreliert sind mit $r=0,79$ bzw. $r=0,80$.

\subsubsection{Diskussion}

Die Untersuchung der Artenzahl-Areal-Beziehung hat in den vergangenen Jahrzehnten nicht nur eine große Anzahl an Funktionen hervorgebracht, die diesen Zusammenhang wiedergeben sollen, sondern in den letzten Jahren auch eine Vielzahl an Kriterien, welche die Approximationsgüte einer Funktion evaluieren können sollen. $\mathrm{Zu}$ den derzeit populärsten zählt das Akaike information criterion (AIC, vgl. Quinn \& Keough 2002), welches z. B. in der Arbeit von FridLey et al. (2005) Anwendung fand. Im folgenden soll zunächst eine kritische Betrachtung der in dieser Arbeit verwendeten Kriterien erfolgen.

Die Kriterien, die in dieser Untersuchung für die Bewertung der hier getesteten Funktionen herangezogen wurden, bieten einfache und direkte Ergebnisse und Vergleichsmöglichkeiten. Dabei stellt das Bestimmtheitsmaß auch in vielen weiteren Arbeiten noch das wichtigste Gütekriterium dar. Alle in dieser Arbeit getesteten Funktionen haben die gleiche Anzahl von Funktionsparametern, so dass ein angepasstes Bestimmtheitsmaß nicht notwendig ist (Quinn \& KeOugh 2002).

Über den relativen Residuenfehler wird ein direkter Vergleich der Schätzwerte mit den empirisch ermittelten Artenzahlen ermöglicht. Die Residuen als Kriterium zu verwenden wäre insofern nicht sinnvoll, als ein Residuum um so größer sein kann, um so größer die ermittelte Artendichte ist, zu der sie in Bezug gesetzt wird. Dass der mittlere relative und der maximale relative Residuenfehler nicht gleichwertig mit den anderen Kriterien zur Evaluation herangezogen werden können, zeigt der hohe Korrelationskoeffizient. Dies zeigt sich in den ermittelten Rängen, die die Funktionen bei diesen Kriterien eingenommen haben. Die Positionen sind bei beiden Kriterien über alle Funktionen die gleichen. Dadurch ist der Informationsgewinn beider Kriterien, wenn man sie getrennt betrachtet nicht groß, bezogen auf diese Untersuchung sogar faktisch nicht vorhanden. Es wäre daher ausreichend mit nur einem dieser beiden Kriterien zu arbeiten. Dabei würde ich den mittleren relativen Residuenfehler dem maximalen vorziehen, weil er die Spanne der relativen Abweichungen wiedergibt und nicht nur einen einzelnen Wert. Dadurch ermöglicht er bessere Rückschlüsse auf die Abweichungen der Schätzwerte von den empirisch ermittelten Artendichten.

Der relative Extrapolationsfehler bezieht sich im Gegensatz zum Bestimmtheitsmaß und dem mittleren bzw. maximalen relativen Residuenfehler nicht auf die Abweichung der Regressionskurve einer Funktion von den empirisch ermittelten Artenzahlen. Er ermöglicht den direkten Vergleich eines mit einer bestimmten Funktion berechneten Schätzwertes einer Artenzahl auf der Fläche A7, nachdem diese Funktion speziell ohne den entsprechenden empirischen Wert approximiert wurde. Die Extrapolation von Artendichten auf größere Flächengrößen, die im Rahmen von Biodiversitätsuntersuchungen nur mit großem Aufwand zu erfassen sind, ist von großem Interesse. Die Simulation solcher Extrapolationen und deren Vergleich mit empirisch erhobenen Daten, daher eine bislang stark vernachlässigte Möglichkeit die Extrapolationsgüte einer Kurve zu beurteilen. Zwar ist die Anpassung einer Regressionskurve mit umso größerer Wahrscheinlichkeit von der Realität entfernt, um so weniger Wertepaare für ihre Berechnung herangezogen werden. Das zeigt sich allein dadurch, dass die Extrapolationen außer bei der linearen Funktion (sowohl in logarithmierter als auch in nicht-logarithmierter Form) in fast allen Fällen deutlich kleiner ausfallen, als der empirisch ermittelte Wert auf dieser Flächengröße. Trotzdem stellt diese Methode eine gute 
Möglichkeit dar die Extrapolationsgüte einer Funktion anhand empirisch erhobener Daten auf kleinen Skalen zu testen.

Insgesamt lässt sich sagen, dass sich mit den hier verwendeten Kriterien ein unkomplizierter und aussagekräftiger Einblick in die Anpassungsgüte verschiedener Funktionen erreicht werden kann.

Viele Funktionen für die Approximation von Artenzahl-Areal-Kurven sind inzwischen postuliert worden und haben intensiven wissenschaftlichen Diskurs hervorgerufen. Eine umfassende Darstellung verschiedener Funktionen findet sich z. B. bei TJøRve (2003), eine umfangreiche Diskussion über die Qualität verschiedener Funktionen findet sich z. B. bei ScheINER (2003, 2004). Die für diese Studie gewählten Funktionen stellen nur eine kleine Auswahl dar, wobei ich mit der Potenzfunktion Arrhenius (1920) und GleAsON (1922) die ältesten und populärsten Vertreter sind. Die lineare Funktion (Connor \& McCoy 1979) und die Michaelis-Menten-Funktion (Schmitт 1999) als Beispiel für eine Sättigungsfunktion finden in der Literatur wenig Verwendung und sollten Extreme, durchschnittlich $\mathrm{zu}$ hoher Approximation einerseits und $\mathrm{zu}$ niedriger Approximation andererseits darstellen.

Anhand der Gütekriterien konnte im Rahmen dieser Arbeit die Potenzfunktion im nichtlogarithmierten Raum als adäquateste Funktion herausgestellt werden. Zu diesem Ergebnis kommen zum Beispiel auch Dolnik (2003, mit Einschränkungen), Drakare et al. 2006 und Dengler (2007, Manuskript für Ecology Letters). In vielen anderen Studien, die sich mit Arten-Areal-Beziehungen beschäftigen wird die Potenzfunktion von vornherein als die passendste Funktion angenommen (z. B. Crawley \& Harral 2001). Sehr populär bei Biodiversitätsuntersuchungen ist allerdings auch die Logarithmusfunktion, die im Rahmen dieser Arbeit keine gute Anpassung an die ArtenzahlAreal-Werte zeigt. Die logarithmierte Form dieser Funktion erreicht beim Ranking der Funktionen zwar den dritten Rang, allerdings mit deutlichem Abstand zu den beiden Funktionen der Potenzfunktion. Dabei wird von einigen postuliert, dass die Logarithmusfunktion auf kleinen Skalen bessere Anpassungen an Arten-Areal-Kurven ermöglicht als die Potenzfunktion (Gleason 1922, Stohlgren et al. 1995, He \& Legendre 1996). Da im Rahmen dieser Arbeit in diesem Sinne nur kleine Skalen untersucht wurden, scheint dies aber nicht stichhaltig zu sein. Die Arbeit von FridLEy et al. (2005) unterstützt dieses Ergebnis. Die Autoren gründen ihre Ergebnisse auf Artendichten von 1472 Plots, in denen ähnliche Flächengrößen untersucht wurden wie in der hiesigen Studie.

Sowohl die Logarithmusfunktion als auch die Sättigungsfunktion zeigen einen asymptotischen Kurvenverlauf, und es gehen viele Autoren davon aus, dass Arten-Areal-Kurven sich ebenfalls asymptotisch einem Sättigungswert nähern (ConNor \& McCoy, He \& Legendre 1996, Schmitt 1999, Lomolino 2000, TJøRve 2003). Jedoch konnten Williamson et al. 2001 anhand Artendichten über eine weite Spanne von Flächengrößen zeigen, dass Arten-Areal-Kurven keine Asymptote haben. Der asymptotische Verlauf der Michaelis-Menten-Funktion ist im Rahmen dieser Studie offensichtlich der Grund für die schlechte Anpassungsgüte dieser Funktion gewesen. Der relative Extrapolationsfehler dieser drei Funktionen liegt bei über $50 \%$. Bei der Logarithmusfunktion im nicht-logarithmierte Raum fällt die extrem hohe Abweichung der Schätzwerte von den empirisch ermittelten Werten auf. Werden die Artenzahl-Areal-Kurven mit logarithmierten Flächengrößen approximiert, so dass für die Logarithmusfunktion eine lineare Regression ermöglicht wird, zeigen die Kurven einen abwärtsgebogenen Verlauf, der sich asymptotisch den kleineren Flächen zu nähern scheint. Die Regressionsgeraden erhalten bei dieser Skalierung einen Verlauf, der auf der linken Seite zum große negative Schätzwerte hervorruft, so dass die Residuen in Relation zu diesen kleinen Artenzahlen stark abweichen. 
Lineare Funktionen werden von keiner aktuellen Studie als geeignet betrachtet, wurden allerdings aufgrund der einfachen Möglichkeit lineare Regressionen anzuwenden angepriesen. So empfehlen Connor \& McCoy (1979) den Gebrauch der Potenzfunktion (in ihrer logarithmierten Form) und anderer linearer Funktionen, aufgrund der guten Vergleichsmöglichkeiten und weil sie in der Vergangenheit und der Gegenwart ihrer Zeit häufig benutzt würden. Lineare Funktionen (abgesehen von der linearisierten Form der Potenzfunktion) würden einer gleichbleibenden Steigung über alle Flächengrößen entsprechen. Dass dies nicht zutreffend ist, wird im nächsten Kapitel noch ausgeführt werden. Aufgrund des starken Anstiegs der Artendichten auf kleinen Flächengrößen, werden lineare Funktionen auf diesen Flächengrößen immer so approximiert werden, dass sie bei Betrachtung größerer Flächen Artendichten vorhersagen, die weit über den tatsächlichen liegen (s. Abb. 26). Dieser Umstand wird eindrucksvoll von den Ergebnissen des relativen Extrapolationsfehlers untermauert, der bei beiden Formen dieser Funktion im Schnitt über $250 \%$ liegt.

\subsection{Zur Steigung der Artenzahl-Areal-Kurven}

\subsubsection{Ergebnisse}

Der z-Wert des Untersuchungsgebietes liegt gemittelt über alle 50 Plots und alle Flächengrößen bei 0,231. Abgesehen vom Übergang der Fläche A1 zur Fläche A2 steigt der durchschnittliche z-Wert mit zunehmender Flächengröße, während die Standardabweichung zunächst fällt, dann aber wieder steigt (Abb. 27).

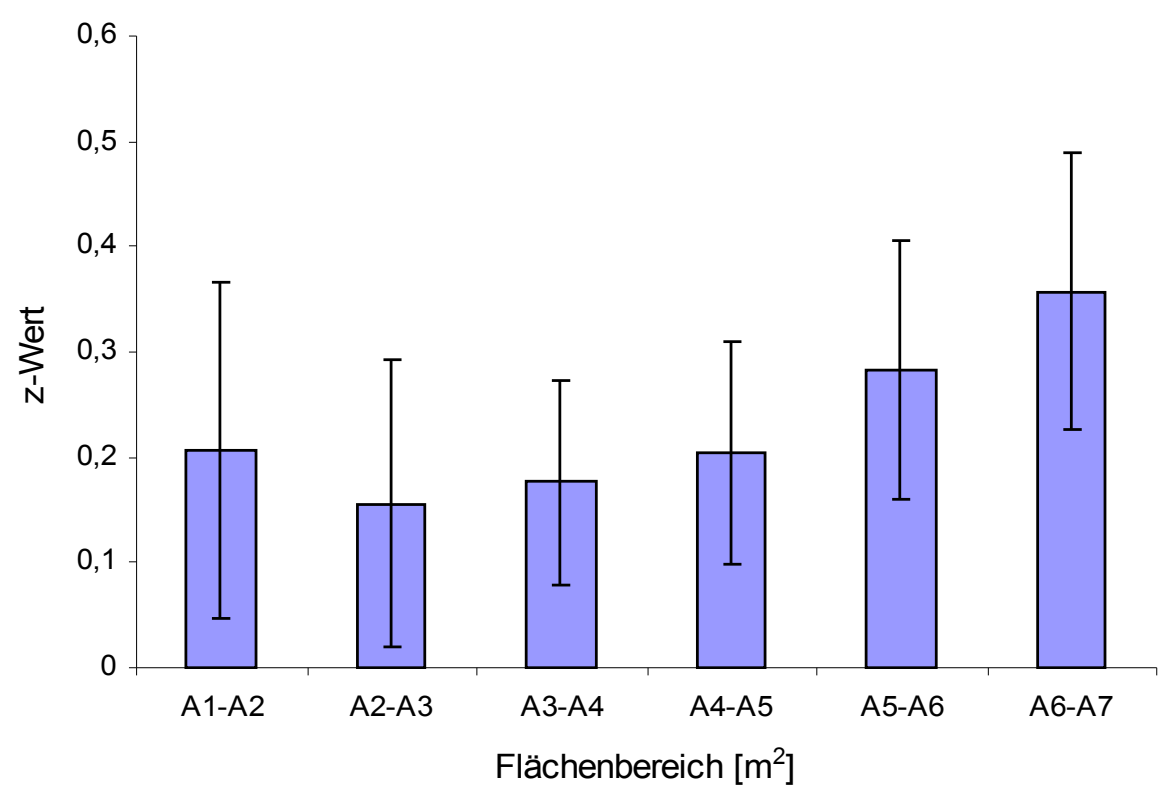

Abbildung 27: Mittlere z-Werte der Gesamtartenzahlen auf allen Plots mit Standardabweichung. 
Der Variationskoeffizient (Standardabweichung im Verhältnis zum arithmetischen Mittel) steigt nur vom ersten zum zweiten Flächenübergang und fällt dann stetig. Um die Unterschiede der Mittelwerte auf Signifikanz zu prüfen, war ein Test der Normalverteilung der Datenreihen notwendig. Schiefe und Kurtosis zeigen zum Teil deutliche Abweichung von einer Normalverteilung. Ein Kolmogorov-Smirnoff-Test zeigt, dass drei der sechs Datenreihen in sich nicht normalverteilt sind. Bei einer logarithmischen Transformation sind vier von sechs Werten normalverteilt und der $\mathrm{p}$-Wert durchschnittlich höher, weshalb ebenfalls mit diesen Werten der Mittelwertvergleich durchgeführt wird.

Die Unterschiede in den Mittelwerten der ersten vier Flächenübergänge sind nach einem post-hocTest auf homogene Gruppen sowohl für die Originalwerte als auch die logarithmisch transformierten Werte nicht signifikant (Tab. 16). So bilden die z-Werte der ersten vier Übergänge eine homogene Gruppe, die nur von den Übergängen A5-A6 und A6-A7 signifikant verschieden sind. Letztere sind bei nicht-transformierten Werten unter sich ebenfalls verschieden, bei transformierten Werten bilden A4-A5 und A5-A6 eine homogene Gruppe, sowie A5-A6 und A6A7.

Tabelle 16: Mittelwert, Schiefe, Kurtosis und p-Wert eines Kolmogorov-Smirnoff-Testes ( $p$ KS) sowie homogene Gruppen der z-Werte $(n=50)$.

\begin{tabular}{lrrrrrr}
\hline & \multicolumn{3}{c}{ Flächenübergang } & & \\
& $A 1-A 2$ & $A 2-A 3$ & $A 3-A 4$ & $A 4-A 5$ & $A 5-A 6$ & $A 6-A 7$ \\
\hline Mittelwert & 0,21 & 0,16 & 0,18 & 0,2 & 0,28 & 0,36 \\
Schiefe & 1,29 & 2,21 & 0,52 & 1,63 & 1,38 & 0,73 \\
Kurtosis & 1,47 & 6,94 & 0,39 & 5,34 & 2,4 & 3,81 \\
p KS & $<0,15$ & $<0,05$ & $>0,20$ & $<0,10$ & $>0,20$ & $>0,20$ \\
homogene Gruppen & $\mathrm{a}$ & $\mathrm{a}$ & $\mathrm{a}$ & $\mathrm{a}$ & $\mathrm{b}$ & $\mathrm{c}$ \\
\hline p KS (log) & $>0,20$ & $>0,20$ & $>0,20$ & $<0,15$ & $>0,20$ & $<0,10$ \\
homogene Gruppen $(\log )$ & $\mathrm{a}$ & $\mathrm{a}$ & $\mathrm{a}$ & $\mathrm{ab}$ & $\mathrm{bc}$ & $\mathrm{c}$ \\
\hline
\end{tabular}

Bei den unterschiedenen Landschaftstypen verlaufen die z-Werte in Abhängigkeit zum Flächenübergang prinzipiell ähnlich zu den z-Werten der Gesamtartenzahlen aller Flächen (Tab. 17). Lediglich der durchschnittliche z-Wert der Artenzahlen der Waldflächen beim Flächenübergang A1-A2 ist nicht größer als die beim Flächenübergang A2-A3. Alle anderen Landschaftstypen zeigen zunächst diesen Rückgang des durchschnittlichen z-Wertes, wonach diese mit zunehmender Flächengröße zunimmt. Dafür sind in keinem der Fälle außer bei den Waldflächen die Mittelwerte der Flächenübergänge signifikant voneinander verschieden, so dass den Änderungen in der Steigung Aussagekraft verloren geht. 
Tabelle 17: Mittlere z-Werte der Artenzahl-Areal-Kurven differenziert nach Landschaftstypen sowie das arithmetische Mittel über alle Flächenübergänge. Die angegeben homogenen Gruppen beziehen sich auf die z-Werte der Artenzahlen auf den Waldflächen.

\begin{tabular}{lccccccc}
\hline & \multicolumn{9}{c}{ Flächenübergang } & & & \\
Landschaftstyp & $A 1-A 2$ & $A 2-A 3$ & $A 3-A 4$ & $A 4-A 5$ & $A 5-A 6$ & $A 6-A 7$ & $\varnothing$ \\
\hline Agrarflächen $(\mathrm{n}=14)$ & 0,211 & 0,112 & 0,178 & 0,179 & 0,225 & 0,309 & 0,202 \\
Grünland $(\mathrm{n}=5)$ & 0,198 & 0,128 & 0,160 & 0,191 & 0,271 & 0,346 & 0,216 \\
Siedlungsflächen $(\mathrm{n}=13)$ & 0,304 & 0,234 & 0,240 & 0,286 & 0,336 & 0,442 & 0,307 \\
Wald $(\mathrm{n}=18)$ & 0,136 & 0,141 & 0,136 & 0,167 & 0,276 & 0,351 & 0,201 \\
\hline homogene Gruppen & $\mathrm{a}$ & $\mathrm{a}$ & $\mathrm{ab}$ & $\mathrm{ab}$ & $\mathrm{bc}$ & $\mathrm{bc}$ & $\mathrm{c}$ \\
\hline
\end{tabular}

Differenziert nach Artengruppen zeigen die durchschnittlichen z-Werte der Gefäßpflanzen und Flechten das gewohnte Bild zunächst steigender, dann fallender Werte. Die z-Werte der Gefäßpflanzen sind bei allen Flächenübergängen normalverteilt. Die homogenen Gruppen zeigen auch hier, dass nicht alle Mittelwerte signifikant voneinander verschieden sind. Bei den Moosen steigen die z-Werte durchgehend. Moose und Gefäßpflanzen zeigen über alle Flächenübergänge gemittelt den gleichen z-Wert. Der durchschnittliche z-Wert über alle Flächenübergänge der Flechten ist doppelt so hoch, was besonders durch die z-Werte der Flächenübergänge A5-A6 und A6-A7 bedingt wird.

Die z-Werte der floristischen Statusgruppen zeigen im Vergleich zu allen anderen Bezugsgruppen die größte Streuung. Während der durchschnittliche z-Wert der indigenen Arten den gewohnten Verlauf zunächst fallender und dann kontinuierlich steigender Werte zeigt, steigen die Werte der Neophyten vom kleinsten zum größten Flächenübergang stetig. Archaeophyten besitzen auf allen Flächenübergängen die höchsten durchschnittlichen z-Werte außer auf dem Übergang A6-A7, wo diese verglichen mit den anderen floristischen Statusgruppen am niedrigsten sind. Über alle Flächengrößen gemittelt sind die z-Werte der Archaeophyten am höchsten, die der kultivierten Arten am niedrigsten.

Tabelle 18: Mittlere z-Werte auf den berücksichtigten Flächenübergängen differenziert nach Artengruppen und floristischen Statusgruppen. Angegeben sind arithmetisches Mittel und sofern vorhanden Standardabweichung sowie das arithmetische Mittel über alle Flächenübergänge $(n=50)$.

\begin{tabular}{lccccccc}
\hline & & \multicolumn{7}{c}{ Fä̈chenübergang } & & & \\
Artengrupppe & $A 1-A 2$ & $A 2-A 3$ & $A 3-A 4$ & $A 4-A 5$ & $A 5-A 6$ & $A 6-A 7$ & $\varnothing$ \\
\hline Gefäßpflanzen & $0,17 \pm 0,12$ & $0,14 \pm 0,12$ & $0,17 \pm 0,10$ & $0,19 \pm 0,11$ & $0,26 \pm 0,11$ & $0,36 \pm 0,13$ & 0,220 \\
homogene Gruppen & $\mathrm{a}$ & $\mathrm{a}$ & $\mathrm{a}$ & $\mathrm{ab}$ & $\mathrm{b}$ & $\mathrm{c}$ & - \\
Moose & $0,13 \pm 0,17$ & $0,17 \pm 0,20$ & $0,19 \pm 0,25$ & $0,20 \pm 0,23$ & $0,27 \pm 0,24$ & $0,33 \pm 0,18$ & 0,217 \\
Flechten & $0,08 \pm 0,16$ & $0,03 \pm 0,12$ & $0,07 \pm 0,15$ & $0,23 \pm 0,35$ & $0,51 \pm 0,41$ & $0,62 \pm 0,32$ & 0,443 \\
\hline Indigene Arten & 0,177 & 0,154 & 0,179 & 0,203 & 0,258 & 0,377 & 0,224 \\
Neophyten & 0,102 & 0,140 & 0,206 & 0,288 & 0,298 & 0,490 & 0,254 \\
Archaeophyten & 0,315 & 0,456 & 0,315 & 0,288 & 0,315 & 0,136 & 0,304 \\
Kultivierte Arten & 0,131 & 0,093 & 0,057 & 0,067 & 0,064 & 0,162 & 0,096 \\
\hline
\end{tabular}




\subsubsection{Diskussion}

Besonders auffällig bei der Betrachtung des Verlaufs der z-Werte scheint zunächst der Rückgang des z-Wertes vom Flächenübergang A1-A2 nach A2-A3. Wie herausgestellt werden konnte ist dieser Unterschied zwar in keinem der untersuchten Fällen signifikant, da zumindest immer die ersten drei Flächenübergänge eine homogene Gruppe gebildet haben. Trotzdem findet sich dieser Rückgang des z-Wertes vom ersten zum zweiten Flächenübergang sowohl bei CRAwLEY \& HarraL (2001) und Chiarucci et al. (2006) als auch bei Dengler \& Allers (2006, s. Tab. 19). Allerdings sind die kleinsten Flächenübergänge dieser Studien auf anderen Flächengrößen, so dass es scheint als wäre der Abfall in der Steigung ein Artefakt des geringsten Flächenübergangs selbst bzw. der Effekte die zu den Artenzahlen dieser Flächen führen.

Tabelle 19: Z-Werte der Gefäßpflanzenartenzahlen in verschiedenen Untersuchungsgebieten bei zufallsveteilten Stichproben, sowie das arithmetische Mittel über die vier Flächen von $0,01 \mathrm{~m}^{2}$ bis $100 \mathrm{~m}^{2}$. (1) Diekmann et al. (2007), (2) Chiarucci et al. (2006), (3) Crawley \& Harral (2001).

\begin{tabular}{lcccc}
\hline & \multicolumn{4}{c}{ Unters uchungsgebiet } \\
Flächenübergang $\left[\mathrm{m}^{2}\right]$ & TK $2728(D)$ & TK $2720 / 2(D)^{1}$ & Bremen $(D)^{2}$ & Berkshire $(U K)^{3}$ \\
\hline $0,0001-0,0009$ & 0,171 & - & - & - \\
$0,0009-0,01$ & 0,142 & - & 0,291 & - \\
$0,01-0,09$ & 0,172 & - & 0,173 & 0,180 \\
$0,09-1$ & 0,192 & - & 0,162 & 0,100 \\
$1-9$ & 0,258 & 0,386 & 0,202 & 0,200 \\
$9-100$ & 0,361 & 0,148 & 0,178 & 0,200 \\
$100-1000$ & - & - & 0,130 & 0,370 \\
$\varnothing$ & 0,246 & - & 0,179 & 0,170 \\
\hline
\end{tabular}

Wurden z-Werte vor zehn Jahren noch über weite Skalenbereiche hinweg oft relativ undifferenziert für größere Regionen angegeben (z. B. Rosenzweig 1995, Новонм 1998), belegen inzwischen immer mehr Autoren die Notwendigkeit einer differenzierten Betrachtung der z-Werte (z. B. CRAWLeY \& Harral 2001, Fridley et al. 2005, Chiarucci et al. 2006). Die Ergebnisse dieser Arbeit unterstützen diese Entwicklung, da der z-Wert auch hier erheblich sowohl über verschiedene Skalenbereiche insgesamt, als auch differenziert nach Landschaftstypen oder Artengruppen stark variiert. Dabei verhalten sich die z-Werte anders auf Inseln als in kontinuierlicher Vegetation des Festlands und wieder unterschiedlich in borealen Nadelwäldern Skandinaviens als im tropischen Regenwald Afrikas (Rosenzweig 1995, Новонм 1998). Neben diesen räumlichen Effekten auf die Arten-ArealKurven gibt es einige andere Faktoren, die die Akkumulation von Arten auf kleinen oder großen Skalen beeinflussen. Wie Williamson (2003) herausstellt, fangen die Einflüsse auf die Ergebnisse, die bei Untersuchungen der Arten-Areal-Kurven und deren Einflussfaktoren erhoben werden, schon bei der Wahl der Methoden bzw. der Art der Datenerhebung an. So werden Artenzahl-ArealErhebungen immer noch auf einer breiten Spanne von Flächengrößen und zum Teil nur für bestimmte Landschaftstypen oder Pflanzengesellschaften erhoben und z-Werte auf unterschiedliche Arten berechnet. Prinzipiell gibt es nach wie vor wenig Arbeiten, die z-Werte anhand empirischer Daten quantifizieren (DraKare et al. 2006). Deshalb sind für die folgenden Vergleiche zum Teil zWerte einiger Studien anhand der angegebenen durchschnittlichen Artenzahlen auf bestimmten Flächengrößen berechnet worden.

Die Variation der z-Werte an sich sowie die variantenreiche Methodik bei der Erfassung der Werte 
macht den z-Wert als Instrument weiterführender Forschung natürlich schwer greifbar. Es gibt bereits einige Arbeiten, die den z-Wert für Flächenbereiche mit größeren Spannen ermitteln, wobei es gängig ist zu unterteilen in kleine Flächenbereiche (kleiner als $100 \mathrm{~m}^{2}$ ), mittlere Flächenbereiche (zwischen $100 \mathrm{~m}^{2}$ und einigen Hektar) und großen Flächenbereiche (größer als $10^{5} \mathrm{~m}^{2}$, z. B. Crawley \& Harral [2001], Fridley et al. [2005], Chiarucci et al. [2006]).

Vergleicht man die z-Werte der Gefäßpflanzenarten der vorliegenden Studie über mehrere Flächengrößen mit denen anderer Autoren, fällt auf, dass im Rahmen dieser Studie für die meisten Flächenübergänge durchweg höhere z-Werte ermittelt wurden (Tab. 19). Weder DiekmanN et al. (2007) noch CHIARUCCI et al. (2006) geben z-Werte für die erfassten Flächengrößen an. In beiden Fällen habe ich eine Berechnung der z-Werte anhand der angegeben durchschnittlichen Artenzahlen durchgeführt. Während CHIARUCCI et al. (2006) sowohl im Untersuchungsgebiet um Bremen als auch in Sienna (Italien) durchschnittlich größere z-Werte auf kleineren Skalen $\left(0,001 \mathrm{~m}^{2}\right.$ bis $\left.10 \mathrm{~m}^{2}\right)$ ermittelten als auf größeren Skalen $\left(0,1 \mathrm{~m}^{2}\right.$ bis $\left.1000 \mathrm{~m}^{2}\right)$, kommen Crawley \& Harral (2001) und Dengler \& Allers (2006) zum gleichen gegensätzlichen Ergebnis wie die vorliegende Studie. Auch sie ermittelten einen Anstieg des z-Wertes über die kleineren Flächen. Allerdings zeigt ein Vergleich der z-Werte, dass CHIARUCCI et al. (2006) für den Flächenbereich zwischen den Flächen A2 und A6 einen mittleren z-Wert ermittelt haben, der demjenigen der vorliegenden Studie für diesen Bereich sehr ähnlich ist. So liegt dieser Wert in Bremen bei 0,198 (0,101 - 0,291) und derjenige dieses Untersuchungsgebietes bei 0,194 (0,049-0,418). Die höhere Streuung der Werte der vorliegenden Untersuchung ist dadurch bedingt, dass CHIARUCCI et al. (2006) für ihre Erhebungen die Plots nur innerhalb eines Landschaftstypes platziert haben. Demnach ist es auch nicht überraschend, dass die z-Werte ihrer Studie auf den größeren Flächen geringer ausfallen als auf den kleineren. Die Homogenität des Bestandes beeinflusst bei Arten-Areal-Erhebungen auch die Steigung der Arten-Areal-Kurve. Новонм (1998) hat die $z$-Werte für verschiedene Pflanzengesellschaften in der räumlich nicht weit vom Untersuchungsgebiet der vorliegenden Studie entfernten Elbtalniederung ermittelt. Dafür hat er die Arten (Gefäßpflanzen, Moose und Flechten) jeder Pflanzengesellschaft für die Flächengrößen A3, A4 und A5 mit jeweils zehn Replikaten erfasst. Über alle untersuchten Pflanzengesellschaften gemittelt ergibt sich ein z-Wert von 0,205. Die z-Werte der vorliegenden Arbeit für diesen Flächenbereich berechnet, liegen leicht darunter mit $\mathrm{z}=0,195$. Bei diesem Vergleich werden allerdings zwei unterschiedliche Untersuchungsgebiet mit in ihren jeweiligen Anteilen völlig unterschiedlichen Pflanzengesellschaften verglichen. DiEKMANN et al. (2007) haben innerhalb eines Untersuchungsgebietes ein Datenset zufallsverteilter Plots und ein weiteres nach den Anforderungen der klassischen Pflanzensoziologie in homogenen Beständen erhoben. Die Berechnung der z-Werte anhand der angegebenen Medianwerte zeigt, dass zufallsverteilte Plots im Schnitt einen höheren z-Wert haben als die nicht-zufallsverteilten, obwohl sie dieser Studie folgend durchschnittlich artenärmer sind.

Die Tatsache, dass z-Werte auch über diese Flächengrößen hinaus steigen, widerspricht der Vorstellung, dass sich Artenzahl-Areal-Kurven bei bestimmten Flächengrößen abflachen und die Artendichten eine Sättigung erreichen (Braun-Blanquet 1952, Dierschke 1994, Lomolino 2000). Die erwartete Abflachung der Artenzahl-Areal-Kurve auf Flächen unterhalb von $1000 \mathrm{~m}^{2}$ einerseits stellt ein Skalierungsproblem der Ordinaten- und Abzissenachse dar (Barkman 1989). In doppeltlogarithmischer Darstellung zeigt sich der Effekt, der in dieser Studie erhobenen Artendichten: die Steigung und damit der Zugewinn an Arten nimmt zu.

Die Abflachung der Kurve auf großen Skalen ist nicht einmal bis zur globalen Betrachtung der 
Arten-Areal-Beziehung zu erwarten, wie WilLiAmson et al. (2001) ausführen. Zwar sei die Artenzahl begrenzt, aber das gleiche gelte auch für die Erdoberfläche. So konnten CRAWLEY \& HARRAL (2001) für den größten in ihrer Studie berücksichtigten Flächenübergang einen z-Wert von 0,208 ermitteln, was bezogen auf Gefäßpflanzenarten sogar noch über dem durchschnittlichen Wert für Flächen bis $100 \mathrm{~m}^{2}$ liegt.

Dass die Artendichten innerhalb kleinerer Flächen mit zunehmender Flächengröße weniger stark steigen als auf größeren, begründen CRAWLEY \& HARRAL (2001) mit den Interaktionen zwischen einzelnen Pflanzenindividuen. Demnach ist der Zugewinn an Arten auf diesen Flächengrößen mit zunehmender Flächengröße gering aufgrund von interspezifischer Konkurrenz und geringen Ausbreitungsraten. Im Rahmen dieser Studie konnten auf den Flächenübergängen A5-A6, sowie A6-A7 innerhalb deren interspezifische Konkurrenz und Ausbreitungsmechanismen eine wichtige Rolle spielen, deutlich höhere z-Wert ermittelt werden als von CRAWLEY \& HARRAL (2001). Deshalb würde ich bei den geringeren z-Werte der Gefäßpflanzenarten eine andere Begründung heranziehen, die Crawley \& Harral (2001) ebenfalls angeführt haben. Da sich die Größe der Pflanzenindividuen nicht proportional zur Flächengröße verhält, ist die Anzahl der Arten auf den kleinen Flächen trotz der Vergrößerung der Flächen um jeweils eine Zehnerpotenz begrenzt. Im Rahmen dieser Arbeit konnte der starke Zugewinn an Arten auf den Flächen A6 und A7 schon mehrfach festgestellt werden.

Mögliche Erklärungen für die unterschiedlichen z-Werte und deren Verlauf mit steigender Flächengröße bieten sich allerdings viele. CHIARUCCI et al. (2006) fanden eine positive Korrelation der z-Werte mit der Biomasse, welche ein Maß der Produktivität darstellt. Mit steigender Biomasse würden demnach die Werte der Kurvensteigung kleiner werden. Das treffe allerdings nur für die zWerte über den gesamten Skalenbereich oder über kleine Skalenbereiche zwischen $0,001 \mathrm{~m}^{2}$ und $10 \mathrm{~m}^{2} \mathrm{zu}$. Die Autoren schließen daraus, dass die Steigung der Artenzahlen auf Flächen mit hoher Produktivität generell niedriger ist. Die Produktivität kann außerhalb von Pflanzenbeständen ausschließlich krautiger Vegetation nicht gut über die Biomasse gemessen werden, weil dort das Sukzessionsstadium die Biomasse wesentlich mitbestimmt (AARSEN et al. 2003). Dass die Steigung innerhalb der Gefäßpflanzenarten auf den Grünlandflächen der vorliegenden Studie größer ist, als die von ChIARUCCI et al. (2006), würde demnach für eine geringere Produktivität der Bestände auf diesen Flächen sprechen.

Sind die Artenzahlen von Dengler \& Allers (2006) über alle Flächengrößen höher als die der vorliegenden Studie (s. Kap. 4.1.2), trifft dies auf die z-Werte nicht zu (Tab. 20). Auf drei von fünf vergleichbaren Flächenübergängen wurden mit dieser Arbeit höhere z-Werte ermittelt, darunter die der größten Flächenübergänge A5-A6 und A6-A7. Das könnte entsprechend der Diskussion unter Kap. 4.1.2 daran liegen, dass die Aufnahmeflächen kleinräumig heterogener in ihren Strukturen waren, so dass sich in den Flächen A7 die Serien der Flächen A1 bis A6 auf zum Teil stark unterschiedlichen Nutzungsformen mit jeweils sehr unterschiedlicher Artenzusammensetzung befanden. 
Tabelle 20: Mittlere z-Werte der Gesamtartenzahlen in verschiedenen Untersuchungsgebieten bei zufallsveteilten Stichproben.

\begin{tabular}{lccccc}
\hline & \multicolumn{5}{c}{ Flächenübergang } \\
Untersuchungsgebiet & $A 2-A 3$ & $A 3-A 4$ & $A 4-A 5$ & $A 5-A 6$ & $A 6-A 7$ \\
\hline TK 2728 $(\mathrm{n}=50)$ & 0,156 & 0,177 & 0,205 & 0,282 & 0,357 \\
TK 3049* $(\mathrm{n}=16)$ & 0,178 & 0,172 & 0,231 & 0,234 & 0,346 \\
\hline
\end{tabular}

* Dengler \& Allers (2006)

Anhand der Daten anderer Studien und der Ergebnisse dieser Arbeit lässt sich insgesamt feststellen, dass die z-Werte auf kleinen Skalen mit zunehmender Flächengröße steigen und in Mitteleuropa über mehrere Skalen hinweg bis $100 \mathrm{~m}^{2}$ bei Gefäßpflanzen einen Wert von durchschnittlich 0,2 $(0,18-0,22)$ annehmen. 


\section{Häufigste Arten und Häufigkeitsverteilungen}

Dieses Kapitel enthält eine umfassende Darstellung der häufigsten Arten auf den unterschiedlichen Flächengrößen. Für die Fläche A7 wird außerdem die Häufigkeitsverteilung dargestellt, mit der die Anzahl häufiger und vergleichsweise seltener Arten visualisiert werden kann.

\subsubsection{Ergebnisse}

Die häufigsten Arten variieren je nach betrachteter Flächengröße, so dass sie differenziert betrachtet werden müssen. Auf allen Flächengrößen außer der kleinsten ist das Moos Brachythecium rutabulum (Krückenförmiges Kurzbüchsenmoos) die häufigste Art (Tab. 21). Auf fünf von sieben Flächengrößen ist Lolium perenne (Deutsches Weidelgras) als damit häufigste Gefäßpflanzenart die zweithäufigste Art insgesamt. Die Differenz der Frequenzen zwischen Brachythecium rutabulum und Lolium perenne beträgt dabei $36 \%$. Damit ist Brachytehcium rutabulum auf 18 Plots mehr vorhanden gewesen als Lolium perenne.

Während auf der Fläche A6 Agrostis capillaris (Rot-Straußgras) die dritthäufigste Art ist, ist die dritthäufigste Art auf der Fläche A7 Holcus lanatus (Wolliges Honiggras), welches sich auf allen anderen Flächen nicht unter den ersten fünf befindet. Agrostis capillaris ist auf der größten Fläche die vierthäufigste Art, womit drei der fünf häufigsten Arten auf allen Plots zu den Süßgräsern gehören. Unter den häufigsten zehn sind es fünf. Es kommen Elymus repens (Gewöhnliche Quecke, siebthäufigste Art) und Festuca rubra agg. (Rot-Schwingel, achthäufigste Art) hinzu.

Es fällt auf, dass Fagus sylvatica und Pinus sylvestris auf der Fläche A1 mit jeweils 20 Registrierungen die häufigsten Arten sind und mit zunehmender Flächengröße aus der Rangliste der fünf häufigsten Arten verschwinden. Quercus robur als dritte Baumart mit großer Abundanz steht auf der kleinsten Flächengröße an dritter Stelle, ist auf den mittleren Flächengrößen nicht mehr unter den ersten fünf, aber dann auf den Flächen A6 und A7 an vierter bzw. fünfter Position der häufigsten Arten. Hypnum cuppressiforme var. Cuppressiforme (Zypressen-Schlafmoos) als zweithäufigstes Moos ist erst auf der Flächen A5 eine der fünf häufigsten Arten. Auf der größten Fläche stellt sie zusammen mit Quercus robur sogar die vierthäufigste (Tab. 21) dar.

Unter den zwanzig häufigsten Arten auf den 50 Plots befindet sich noch ein weiteres Moos. Es handelt sich dabei um Rhytidiadelphus squarrosus (Sparriges Kranzmoos). Dicranoweissia cirrata (Lockiges Gabelzahnperlmoos) nimmt mit einer Frequenz von 13 Registrierungen die Position 24 ein. Neben Lepraria incana zählt keine weitere Flechte zu den 20 häufigsten Arten. Die 21 häufigsten Arten sind indigen, erst an 22. Stelle findet sich mit ebenfalls 13 Registrierungen auf der Fläche A7 der Archaeophyt Veronica arvensis (Feld-Ehrenpreis).

An nächster Stelle kommt (auch 13 mal frequentiert) der häufigste Neophyt, Conyza canadensis. Der häufigste Farn ist Dryopteris carthusiana an Position 33, der auf 11 Plots gefunden wurde. Der häufigste Neophyt auf der kleinsten Flächengröße ist ein Phanerophyt. Es handelt sich um Larix kaempferi, die auf dieser Flächengröße vier mal frequentiert war. Die häufigste kultivierte Pflanze auf allen Flächengrößen war Hordeum vulgare (Saat-Gerste). 
Tabelle 21: Prozentualer Anteil der Frequenz der häufigsten Arten bei zunehmender Flächengröße, sortiert nach den Prozenten der Artenfrequenzen auf der Fläche A7.

\begin{tabular}{lccccccc}
\hline & \multicolumn{6}{c}{$\mathbf{N}=\mathbf{2 0 0}$} & \multicolumn{3}{c}{$\mathbf{N}=\mathbf{5 0}$} \\
Art & 0,0001 & 0,0009 & 0,01 & 0,09 & 1 & 9 & 100 \\
\hline Brachythecium rutabulum & 8 & 15 & 23,5 & 33 & 48,5 & 65 & $\mathbf{8 4}$ \\
Lolium perenne & 7 & 12 & 13 & 16,5 & 21 & 27,5 & $\mathbf{4 8}$ \\
Holcus lanatus & 2,5 & 4 & 4,5 & 8,5 & 14 & 21,5 & $\mathbf{4 6}$ \\
Agrostis capillaris & 5 & 6,5 & 12 & 15,5 & 16,5 & 25,5 & $\mathbf{4 4}$ \\
Hypnum cupressiforme var. cup. & 2,5 & 3 & 4,5 & 7,5 & 14,5 & 24 & $\mathbf{4 4}$ \\
Quercus robur & 7,5 & 8 & 8,5 & 8,5 & 13,5 & 24 & $\mathbf{4 4}$ \\
Elymus repens & 3,5 & 5 & 7,5 & 12,5 & 19 & 25 & $\mathbf{4 2}$ \\
Festuca rubra agg. & 6 & 10,5 & 14 & 15 & 16,5 & 23,5 & $\mathbf{4 2}$ \\
Taraxacum spec. & - & 1 & 1,5 & 3,5 & 7 & 18,5 & $\mathbf{4 0}$ \\
Lepraria incana & - & - & - & - & - & 6,5 & $\mathbf{4 0}$ \\
\hline Stellaria media & 1 & 1,5 & 4 & 6,5 & 12 & 23 & 38 \\
Scleropodium purum & 3 & 5 & 6,5 & 10 & 14,5 & 18,5 & 34 \\
Pinus sylvestris & 10 & 12 & 12,5 & 13,5 & 14,5 & 19 & 28 \\
Fagus sylvatica & 10 & 10,5 & 11 & 11 & 12,5 & 19,5 & 22 \\
Pleurozium schreberi & 3,5 & 5 & 6 & 7 & 9 & 10,5 & 18 \\
Hordeum vulgare & 3,5 & 5,5 & 6 & 6,5 & 7 & 8,5 & 10 \\
Brassica napus & 4 & 5 & 6 & 6 & 6 & 6,5 & 8 \\
\hline
\end{tabular}

Die Frequenzen der häufigsten Arten entwickeln sich mit zunehmender Flächengröße zum Teil ganz unterschiedlich (Abb. 28). Die Verteilung der Häufigkeiten der Arten zeigt einen starken Abfall derer, die auf 1-10\% der Plots zu finden waren und derjenigen, die sich auf $11-20 \%$ der Plots befanden (Abb. 28). Die Sprünge danach fallen geringer aus, wobei auffällt, dass mehr Arten auf $41-50 \%$ der Plots zu finden waren als auf 31-40\%. Nur Brachythecium rutabulum kam auf mehr als $50 \%$ aller Plots vor. Fast Die Hälfte aller registrierten Arten (290 von 585) kam nur auf einem Plot vor, weitere 92 lediglich auf zwei Plots (Abb. 29).

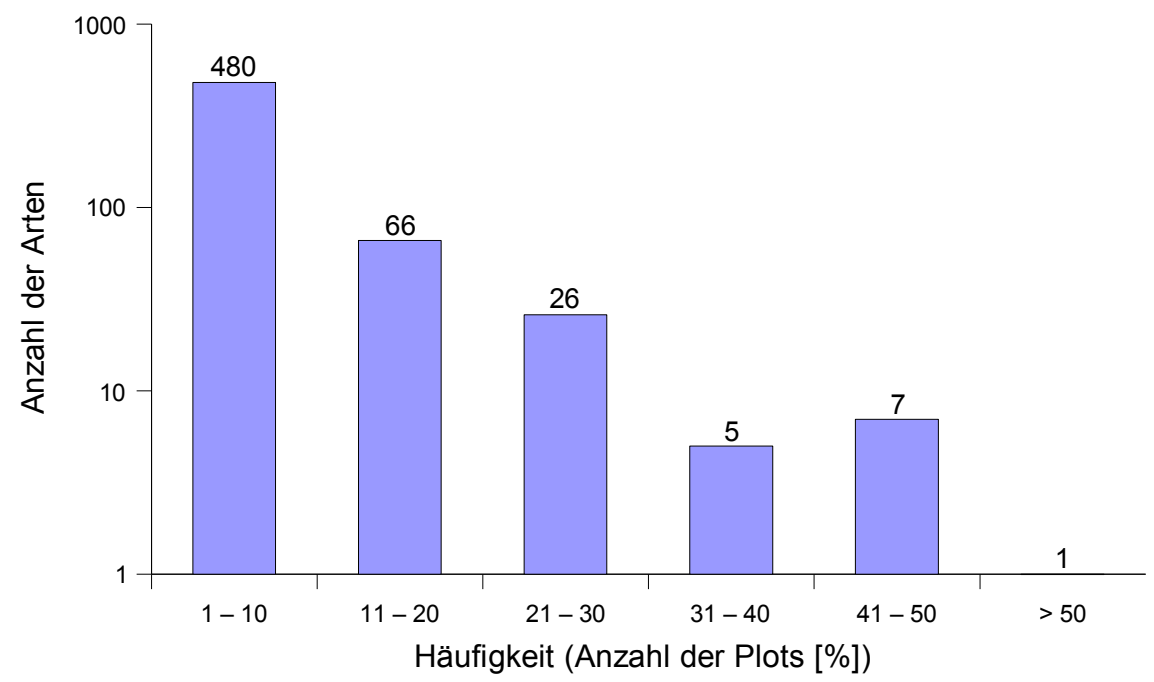

Abbildung 28: Häufigkeitsverteilung der Arten im Untersuchungsgebiet bei logarithmierter Skalierung der Ordinatenachse. 


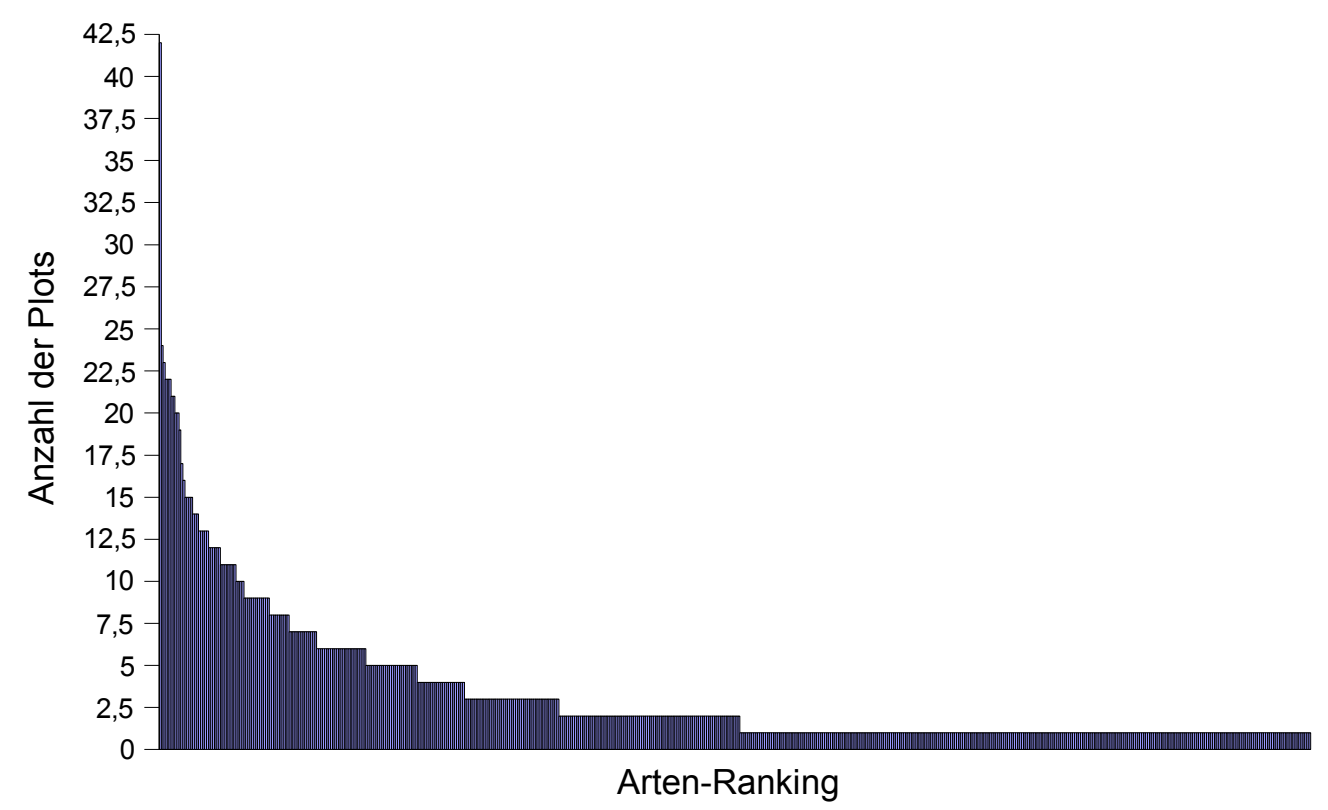

Abbildung 29: Muster der Häufigkeitsverteilung der Arten auf den 50 Plots.

Die Frequenzen aller Arten auf den Flächen A6 und A7 sind im Anhang 1 zu finden.

\subsubsection{Diskussion}

Bei einer zufälligen Stichprobenverteilung, bei der viele unterschiedliche Pflanzengesellschaften beprobt werden, sind zwangsläufig die Arten am häufigsten, die eine ubiquitäre Verbreitung aufweisen und in ihren Standortansprüchen euryök sind, also im Bezug auf abiotische Umweltfaktoren über eine große Toleranzspanne verfügen.

In einer bereits zitierten Studie von Dengler \& Allers (2006) im Osten Brandenburgs konnte ebenfalls Brachytehcium rutabulum als häufigste Art innerhalb der Fläche A7 nachgewiesen werden (Tab. 22). Der Unterschied in der prozentualen Häufigkeit von $3 \%$ ist sehr geringfügig. Bei Lolium perenne, der zweithäufigsten Art der vorliegenden Untersuchung, unterscheidet sich die prozentuale Häufigkeit mit der von Dengler \& Allers (2006) ermittelten ebenfalls kaum. Allerdings ist Lolium perenne in Brandenburg nicht die zweithäufigste Art gewesen. Außerdem beträgt der prozentuale Rückgang von der häufigsten zur zweithäufigsten Art nur $18 \%$, also die Hälfte weniger als dieser Unterschied im hier untersuchten Gebiet beträgt. Hypnum cupressiforme var. cupressiforme, Elymus repens ssp. repens, Stellaria media, Taraxacum spec. und Impatiens parviflora waren in Brandenburg prozentual höher frequentiert als Lolium perenne. Damit konnte mit Hypnum cupressiforme var. cupressiforme als zweithäufigste Art in Brandenburg ein weiteres Moos ermittelt werden. Diese Art kommt laut Schulz \& Dengler (2006) epiphytisch, epilithisch, epigäisch und epixyl vor und ist kosmopolitisch verbreitet. Im Rahmen der vorliegenden Untersuchung war das Vorkommen von Hypnum cupressiforme var. cupressiforme hingegen weitestgehend auf Wälder beschränkt. Es kam sowohl epigäisch, epiphytisch als auch epixyl vor, nicht jedoch epilithisch. Die Frequenz dieser Art war in Brandenburg um fast ein Drittel höher. Das 
deutlich höhere Vorkommen dieser Art in Brandenburg kann darin begründet sein, dass in dieser Studie Standorte, die Hypnum cupressiforme var. cupressiforme nicht besiedelt (z. B. Äcker), einen geringeren Flächenanteil haben und damit auch weniger erfasst wurden. So befanden sich acht der sechzehn Plots, die Dengler \& Allers (2006) untersuchten, in Wäldern, drei auf Äckern, vier auf Grünland und nur einer in einem Garten. Das erklärt die im Vergleich höheren Frequenzen von Hypnum cupressiforme var. cupressiforme, Impatiens parviflora, Fagus sylvatica, Lophocolea heterophylla, Dicranella heteromalla und Pinus sylvestris. Während Fagus sylvatica und Pinus sylvestris als Baumarten erwartungsgemäß am häufigsten auf Waldflächen wachsen, sind die Standort von Impatiens parviflora laut JäGER \& WERNER (2005) vor allem Waldsäume, Waldwege, Gebüsche, Hecken sowie Laub- und Nadelholzforste. Alle diese Arten sind im Rahmen der hiesigen Untersuchung häufiger in Wäldern bzw. an Waldrändern nachgewiesen worden als auf allen anderen Landschaftstypen.

Tabelle 22: Frequenzen der 20 häufigsten Arten der vorliegenden Studie im Vergleich mit den Angaben von Dengler \& Allers (2006) in Brandenburg mit $\mathrm{AG}=$ Artengruppe, $\mathrm{FS}=$ floristische Statusgruppe und $\mathrm{F}=$ Frequenz [\%].

\begin{tabular}{llllllll}
\hline \multicolumn{1}{c}{ TK 2728 Lüneburg / Niedersachsen $(\mathbf{n}=\mathbf{5 0})$} & \multicolumn{3}{c}{ TK3049 Groß Ziethen / Brandenburg (n= 16) } \\
Art & $A G$ & $F S$ & $F$ & Art & $A G$ & $F S$ & $F$ \\
\hline Brachythecium rutabulum & M & I & $84 \%$ & Brachythecium rutabulum & M & I & $81 \%$ \\
Lolium perenne & G & I & $48 \%$ & Hypnum cupressiforme var. cup. & M & I & $63 \%$ \\
Holcus lanatus & G & I & $46 \%$ & Elymus repens ssp. repens & G & I & $56 \%$ \\
Agrostis capillaris & G & I & $44 \%$ & Stellaria media & G & I & $56 \%$ \\
Hypnum cupressiforme var. cup. & M & I & $44 \%$ & Taraxacum spec. & G & I & $56 \%$ \\
Quercus robur & G & I & $44 \%$ & Impatiens parviflora & G & N & $50 \%$ \\
Elymus repens & G & I & $42 \%$ & Bryum spec. & M & I & $44 \%$ \\
Festuca rubra agg. & G & I & $42 \%$ & Fagus sylvatica & G & I & $44 \%$ \\
Taraxacum spec. & G & I & $40 \%$ & Lepraria incana & F & I & $44 \%$ \\
Lepraria incana & F & I & $40 \%$ & Loliumperenne & G & I & $44 \%$ \\
Stellaria media & G & I & $38 \%$ & Lophocolea heterophylla & M & I & $44 \%$ \\
Scleropodiumpurum & M & I & $34 \%$ & Poa trivialis ssp. trivialis & G & I & $44 \%$ \\
Dactylis glomerata ssp. glomerata & G & I & $32 \%$ & Urtica dioica & G & I & $44 \%$ \\
Rubus corylifolius agg. & G & I & $30 \%$ & Cerastium holosteoides & G & I & $38 \%$ \\
Sorbus aucuparia & G & I & $30 \%$ & Chenopodium album & G & A/I & $38 \%$ \\
Poa trivialis ssp. trivialis & G & I & $30 \%$ & Dicranella heteromalla & M & I & $38 \%$ \\
Poa angustifolia & G & I & $30 \%$ & Pinus sylvestris & G & I & $38 \%$ \\
Pinus sylvestris & G & I & $28 \%$ & Plantago major & G & I & $38 \%$ \\
Galium aparine & G & I & $28 \%$ & Polygonum aviculare agg. & G & I & $38 \%$ \\
Rhytidiadelphus squarrosus & M & I & $28 \%$ & Rubus idaeus & G & I & $38 \%$ \\
\hline
\end{tabular}

Thompson et al. (2004) konnten Poa trivialis als häufigste Art und Eurhynchium praelongum als häufigstes Moos in britischen Gartenfloren ermitteln. Die prozentuale Frequenz dieses Mooses (83\%) unterscheidet sich ebenfalls kaum von der, die Brachythecium rutabulum in der vorliegenden Studie oder bei Dengler \& Allers 2006 erreichte. Damit war in allen drei Untersuchungen zumindest eine Moosart mit mehr als $80 \%$ im jeweiligen Untersuchungsgebiet frequentiert. Brachythecium rutabulum war in der britischen Studie mit $56 \%$ allerdings deutlich weniger frequentiert, als in den deutschen Untersuchungsgebieten. Das ist insofern überraschend, als dass ThOMPson et al. (2004) nur Rasenflächen in Gärten untersucht haben, in denen bei dieser Untersuchung Rhytidiadelphus squarrosus das häufigste Moos darstellt, während Eurhynchium praelongum hauptsächlich in Wäldern zu finden war. 
Betrachtet man die Häufigkeitsangaben der Gefäßpflanzenarten in ElLENBERG et al. (1991), fallen außer Impatiens parviflora alle Gefäßpflanzenarten der 20 häufigsten Arten in Brandenburg bei der Messtischblattfrequenz in die höchste Kategorie 9 (Tab. 23). Das bedeutet, dass diese Arten auf $75 \%$ aller Rasterfelder Deutschlands zu finden sind. Für die häufigsten Gefäßpflanzenarten dieser Studie gilt selbiges. Allerdings ist diese auf das gesamte Bundesgebiet bezogene Angabe wenig aussagekräftig. Die lokale Dominanz einer Art gibt einen besseren Anhaltspunkt für die Frequenz einer Art innerhalb der Untersuchungsgebiete der hier verglichenen Studien. Dieser Wert reicht ebenfalls von 1 (stets vereinzelt auftretend) bis 9 (fast immer in großen Herden flächendeckend) (Ellenberg et al. 1991). Diese Angabe soll „eine Vorstellung von der lokalen Bestandsdichte geben“ (ElLENBERG et al. 1991) und ist dadurch auch nur eingeschränkt als Referenz für die ermittelten Arthäufigkeiten verwendbar. Allerdings mögen diese Angaben sehr wohl als Anhaltspunkt für lokale Häufigkeiten dienen. Elymus repens ssp repens, welches sowohl bundesweit sehr häufig ist als auch lokal in großen Herden flächendeckend auftritt, wurde bei der vorliegenden Studie im Vergleich zu Dengler \& Allers (2006) nur selten erfasst. Sorbus aucuparia hingegen, welches nach ElLENBERG et al. (1991) örtlich nur in geringen Beständen auftritt, belegt beim Artenranking einen vergleichsweise hohen Rang. Bei diesen Vergleichen ist zu beachten, dass die prozentualen Frequenzen der häufigsten Arten bei Dengler \& Allers (2006) insgesamt deutlich höher sind.

Tabelle 23: Die häufigsten Gefäßpflanzenarten der vorliegenden Untersuchung und in Brandenburg Dengler \& Allers (2006) entsprechend, mit deren Rang (R), Messtischblattfrequenz (M), Dominanz im Gelände (D).

\begin{tabular}{|c|c|c|c|c|c|c|c|}
\hline \multicolumn{4}{|c|}{ TK2728 Lüneburg / Niedersachsen $(n=50)$} & \multicolumn{4}{|c|}{ TK3049 Groß Ziethen / Brandenburg $(n=16)$} \\
\hline$R$ & Art & $M$ & $D$ & $R$ & Art & $M$ & $D$ \\
\hline 2 & Loliumperenne & 9 & 6 & 3 & Elymus repens ssp. repens & 9 & 9 \\
\hline 3 & Holcus lanatus & 9 & 6 & 4 & Stellaria media & 9 & 6 \\
\hline 4 & Agrostis capillaris & 9 & 6 & 5 & Taraxacum spec. & 9 & 4 \\
\hline 6 & Quercus robur & 9 & 6 & 6 & Impatiens parviflora & 7 & 5 \\
\hline 7 & Elymus repens ssp. repens & 9 & 9 & 8 & Fagus sylvatica & 9 & 9 \\
\hline 8 & Festuca rubra agg. & 9 & 7 & 10 & Lolium perenne & 9 & 6 \\
\hline 9 & Taraxacumspec. & 9 & 4 & 12 & Poa trivialis ssp. trivialis & 9 & 6 \\
\hline 10 & Stellaria media & 9 & 6 & 13 & Urtica dioica & 9 & 6 \\
\hline 12 & Dactylis glomerata ssp. glomerata & 9 & 5 & 14 & Cerastium holosteoides & - & - \\
\hline 13 & Rubus corylifolius agg. & - & - & 15 & Chenopodium album & 9 & 4 \\
\hline 14 & Sorbus aucuparia & 9 & 3 & 17 & Pinus sylvestris & 9 & 7 \\
\hline 15 & Poa trivialis ssp. trivialis & 9 & 6 & 18 & Plantago major & 9 & 4 \\
\hline 16 & Poa angustifolia & - & - & 19 & Polygonum aviculare agg. & 9 & 4 \\
\hline 17 & Pinus sylvestris & 9 & 7 & 20 & Rubus idaeus & 9 & 5 \\
\hline 18 & Galium aparine & 9 & 4 & & & & \\
\hline
\end{tabular}

Die Verteilung der Artenzahlen zeigt ein typisches Muster für Pflanzengesellschaften mit einer geringen Anzahl häufiger Arten und einer hohen Anzahl seltener Arten (Grime 1998). Dass bei einer zufälligen Stichprobenverteilung Arten mit ubiquitärer Verteilung, wie Brachytehcium rutabulum besonders häufig sind, entspricht bei einer zufälligen Stichprobenverteilung ebenfalls den Erwartungen. In homogenen Beständen ist die Artenzusammensetzung relativ konsistent. Natürlich kann je nach betrachteter Pflanzengesellschaft die Anzahl häufiger Arten und seltener Arten variieren. Trotzdem bleiben diese Verhältnisse erwartungsgemäß gleich. Eine Art die an einem Standort einer Pflanzengesellschaft häufig ist, ist an einem anderen Standort ebenfalls häufig. 
Bei einer zufälligen Stichprobenverteilung im Umfang der vorliegenden Studie, bei der viele verschiedene Pflanzengesellschaften beprobt werden, sind die Arten, die zur Artenzusammensetzung vieler Gesellschaften gehören, die häufigsten. So registrierten DieKMANN et al. (2007) auf zufallsverteilten Plots ebenfalls fast $40 \%$ der Arten nur auf einem Plot, jeweils $20 \%$ der Arten auf zwei bzw. drei Plots. Mit steigender Anzahl Plots fällt die Anzahl registrierter Arten, die auf ihnen allen registriert wurden. So war keine Art auf $30 \%$ der Plots, $2 \%$ aller Arten waren auf $33 \%$ der Plots. Dieser Verlauf entspricht den in dieser Untersuchung festgestellten Artenhäufigkeiten (Abb. 28). 


\section{Zusammenfassung}

Im Rahmen dieser Diplomarbeit wurde die durchschnittliche Pflanzenartenvielfalt der mitteleuropäischen Kulturlandschaft auf verschiedenen räumlichen Skalenebenen insgesamt und differenziert nach Landschaftstypen und Artengruppen untersucht. Durch Berücksichtigung in der Literatur bislang weitestgehend vernachlässigter Aspekte bei der Erhebung von Artenzahl-ArealDaten konnten dabei einige Lücken geschlossen und deren Bedeutung durch ein großes Datenset herausgestellt oder relativiert werden.

Das Untersuchungsgebiet entsprach dem Messtischblatt 2728 (Lüneburg) der TK 25. In diesem ca. $126 \mathrm{~km}^{2}$ großen Gebiet finden sich zu ca. $35 \%$ Waldflächen, 30 \% Agrarflächen, 30 \% Siedlungsund Industrie- bzw. Gewerbefläche und zu $10 \%$ Grünlandflächen. Das gesamte Gebiet ist sehr flach und hat subatlantisches, gemäßigtes Klima. Die Florenausstattung ist entsprechend des Übergangscharakters des Gebietes von ozeanischen zu kontinentalen Bedingungen üppig mit bislang 797 registrierten Gefäßpflanzenarten.

Innerhalb dieses Gebietes wurden 50 Plots absolut zufällig und ungeachtet der Homogenität der Vegetation platziert. Die Koordinaten dieser Plots wurden dazu per Zufallsgenerator generiert und anschließend mit einem GPS-Empfänger aufgesucht. Die Plots waren $100 \mathrm{~m}^{2}$ groß und enthielten jeweils vier Serien von Subplots folgender Flächengrößen: 0,0001 $\mathrm{m}^{2}, 0,0009 \mathrm{~m}^{2}, 0,01 \mathrm{~m}^{2}, 0,09 \mathrm{~m}^{2}$, $1 \mathrm{~m}^{2}$ und $9 \mathrm{~m}^{2}$. Auf allen Flächengrößen wurden die Arten der Gefäßpflanzen, Moose und Flechten erfasst.

Auf einer Fläche von $1 \mathrm{~cm}^{2}$ konnte eine durchschnittliche Artendichte von 1,63 Arten festgestellt werden, auf $100 \mathrm{~m}^{2}$ waren es durchschnittlich 38,7. Das ist mehr als in ähnlichen Studien westlich des Untersuchungsgebietes ermittelt wurde. In einer östlich des Untersuchungsgebites durchgeführten Studie fanden sich hingegen durchschnittlich mehr Arten. Hier machen sich Effekte der Kontinentalität bemerkbar. Die höchste Artendichte von 137 auf $100 \mathrm{~m}^{2}$ befand sich auf einer Siedlungsfläche. Siedlungsflächen waren auch durchschnittlich am artenreichsten, was unter anderem am menschlichen Eingreifen in die Artenzusammensetzung sowie der großen innerstädtischen Strukturheterogenität liegt. Die im Vergleich zu den $9 \mathrm{~m}^{2}$-Flächen sehr hohe Artendichte auf den $100 \mathrm{~m}^{2}$-Flächen, die durch den hohen mittleren z-Wert zwischen diesen Flächen bestätigt wird, ist als Effekt der Stichprobenverteilung zu betrachten. In Kulturlandschaften finden sich besonders in und um Städten sehr heterogenen Strukturen, die umso wahrscheinlicher erfasst werden, je größer die Aufnahmefläche ist.

Moose machen dieser Studie zufolge auf $100 \mathrm{~m}^{2} 15 \%$ der Artendichte aus, Flechten $11 \%$. Die Anteile aller Artengruppen schwanken dabei stark über die verschiedenen Flächengrößen und die unterschiedenen Landschaftstypen (Agrarflächen, Grünland, Siedlungsflächen und Wald). Flechten waren im Vergleich zu Moosen stärker an das Vorkommen bestimmter Substrate gebunden. Der kontinuierliche Anstieg der mittleren Anteile der Flechten bei zunehmender Flächengröße bestätigt dies. Insgesamt machen Moose und Flechten einen wesentlichen Anteil der Phytodiversität aus und sollten daher in vegetationskundlichen Arbeiten nicht vernachlässigt werden. Für die Untermauerung dieser These wären weiterführend Korrelationen der Grfäßpflanzendichten und der Nicht-Gefäßpflanzendichten sinnvoll. Bezüglich der untersuchten floristischen Statusgruppenzeigte sich, dass kultivierte Arten erwartungsgemäß auf Agrarflächen beschränkt. Neophyten haben den größten Anteil auf den $100 \mathrm{~m}^{2}$-Flächen, wobei sie durchschnittlich am häufigsten auf den Siedlungsflächen sind, wo sie überwiegend aus Gartenpflanzen bestehen. Außerdem ist die 
Neophytenzahl positiv mit der Gesamtartenzahl korreliert. Dadurch kann bestätigt werden, dass Neophyten nicht per se durch menschliche Eingriffe gefördert wird.

Bei der Beschreibung der Artenzahl-Areal-Beziehung zeigte die Potenzfunktion die beste Anpassungsgüte. Sie schnitt bei allen verwendeten Kriterien deutlich besser ab als die Logasrithmusfunktion, mit der bei der Approximation aufgrund des scheinbar exponentiellen Verlaufs der Artenzahl-Areal-Kurven bei logarithmierter Flächengröße hohe negative Schätzwerte auf kleinen Flächen entstanden. Sogar die Michaelis-Menten-Funktionschnitt, die bislang in wenigen Studien favorisiert wurde, schnitt durchschnittlich besser ab als die Logarithmusfunktion. Die Ergebnisse dieser Arbeit mit denen anderer Studien zu vergleichen ist schwierig, da sich die Erhebungsmethoden zum Teil stark unterscheiden und es vom Aufnahmedesign bis zur Datenauswertung viele Effekte gibt, die die Ergebnisse beeinflussen.

Der z-Wert variiert je nach betrachtetem Flächenabschnitt, Landschaftstyp oder Artengruppe zum Teil stark. Er liegt über alle Plots gemittelt bei 0,231, was im Vergleich zu anderen Studien einen hohen Wert für diesen Flächenbereich darstellt. Der Verlauf der mittleren z-Werte über die untersuchten Flächengrößen zeigt einen nahezu kontinuierlichen Anstieg und liegt beim größten Flächenübergang mit $\mathrm{z}=0,361$ deutlich über dem Mittelwert, was den Ergebnissen vieler anderen Arbeiten widerspricht. Vergleichsmöglichkeiten dieser Werte sind allerdings gering, da es auch hier an einheitlichen Methoden fehlt.

Häufigste Art über alle Flächen außer der kleinsten war das Moos Brachythecium rutabulum. Es kommt im Untersuchungsgebiet epigäisch, epiphytisch und epixyl vor und hat eine deutlich höhere Frequenz auf $100 \mathrm{~m}^{2}$ als die zweithäufigste Art Lolium perenne. 


\section{Literatur}

Aarsen, L. W., Laird, R. A., Pither, J. (2003): Is the productivity of vegetation plots higher or lower when there are more species? Variable predictions from interaction of the sampling effect and competitive dominance effect on the habitat templet. - Oikos 102: 427-432, Lund.

Ackermann, W., Durka, W. (1998): SORT 4.0 - Programm zur Bearbeitung von Vegetationsaufnahmen und Artenlisten - Handbuch. - 138 S., Mskr., München [u. a.].

ArrhenIus, O. (1921): Species and area. - Journal of Ecology 9: 95-99.

ArrhenIUs, O. (1922): Distribution of the species over the area. - Medd. K. Vetenskapsakad. Nobelinst. 4(7): $1-6$, Uppsala [u. a.].

Barkman, J. J., Doing, H., Segal, S. (1964): Kritische Bemerkungen und Vorschläge zur quantitativen Vegetationsanalyse. - Acta Bot. Neerl. 13: 394-419, Oxford [u. a.].

Barkman, J. J. (1989a): A critical evaluation of minimum area concepts. - Vegetatio 85: 105-116, Dordrecht.

Benkert, D., Fukarek, F., Korsch, H. (1996) [Hrsg.]: Verbreitungsatlas der Farn- und Blütenpflanzen Ostdeutschlands. -615 S., Fischer, Jena [u. a.].

Bосн, S. (2005): Phytodiversität, Charakterisierung und Syntaxonomie der Trockenrasen auf Saaremaa (Estland). - II + 89 + IX S., Beilagemappe, Diplomarb., Institut für Ökologie und Umweltchemie, Univ. Lüneburg.

Boess, J. (1997): Böden. - Cordes, H., Kaiser, T., Lancken, H. von der, Lütkepohl, M., Prüter, J. [Hrsg.]: Naturschutzgebiet Lüneburger Heide - Geschichte - Ökologie - Naturschutz: 29-32, Verl. H. M. Hauschild, Bremen.

Bohn, U., Gollub, G., Hettwer, C. (2000): Karte der natürlichen Vegetation Europas - Maßstab $1: 2500000$ - Legende. - 153 S., Bundesamt für Naturschutz, Bonn.

Bohn, U., Gollub, G., Hettwer, C., Neuhäuslová, Z., Raus, T., Schlüter, H., Weber, H., Hennekens, S. (2004) [Hrsg.]: Karte der natürlichen Vegetation Europas - Maßstab $1: 2500000$ - Erläuterungstext, Legende, Karten [dt./engl.]. - CD-ROM + 19-seitiges Booklet, Bundesamt für Naturschutz, Bonn.

Bohn, U., Gollub, G., Hettwer, C., Neuhäuslová, Z., Schlüter, H., Weber, H. (2003) [Hrsg.]: Karte der natürlichen Vegetation Europas - Maßstab 1:2 500000 - Erläuterungstext. - 655 S., 13 Kt., CD-ROM, Bundesamt für Naturschutz, Bonn.

Boнn, U., NeuhäusL, R. (2000) [Hrsg.]: Karte der natürlichen Vegetation Europas - Maßstab $1: 2500000$ Karten. - 10 Kt., 1 Legende, Bundesamt für Naturschutz, Bonn.

Braun-Blanquet, J. (1951): Pflanzensoziologie - Grundzüge der Vegetationskunde. - 2. Aufl., XI + 631 S., Springer, Wien.

Bundesamt Für Naturschutz (1996) [Hrsg.]: Rote Listen gefährdeter Pflanzen Deutschlands. Schriftenr. Vegetationskd. 28: 744 S., Bonn.

Chiarucci, A., Viciani, D., Winter, C., Diekmann, M. (2006): Effects of productivity on species-area curves in herbaceous vegetation: evidence from experimental and observational data. - Oikos, Lund.

Conert, H. J. (2000): Pareys Gräserbuch. - 592 S., Blackwell Wissenschafts-Verl., Berlin [u. a.].

Connor, E. F., McCoy, E. D. (1979): The statistics and biology of the species-area relationship. - Am. Nat. 113: 791-833, Chicago, Ill.

Crawley, M. J. (1997): Biodiversity. - Crawley, M. J. [Hrsg.]: Plant Ecology. - 2. Aufl.: 595-632, Blackwell, Malden, MA [u. a.].

Crawley, M. J. (1997) [Hrsg.]: Plant Ecology. - 2. Aufl., XVII + 717 S., Blackwell, Malden, MA [u. a.].

Crawley, M. J., Harral, J. E. (2001): Scale Dependence in Plant Biodiversity. - Science 291: 864-868, Washington, DC.

Csapody, V. (1968): Keimlingsbestimmungsbuch der Dikotyledonen. - 286 S., Akademiai Kiado, Budapest.

Dengler, J. (2003): Entwicklung und Bewertung neuer Ansätze in der Pflanzensoziologie unter besonderer Berücksichtigung der Vegetationsklassifikation. - Arch. Naturwiss. Diss. 14: 297 S., Galunder, Nümbrecht.

Dengler, J. (2005): Zwischen Estland und Portugal - Gemeinsamkeiten und Unterschiede der 
Phytodiversitätsmuster europäischer Trockenrasen. - Tuexenia 25: 387-405, Göttingen.

Dengler, J. (2006): Variabilität von Artendichte und Artenzusammensetzung auf unterschiedlichen räumlichen Skalenebenen - Exemplarische Untersuchungen aus Trockenrasen und Konsequenzen für das Probedesign von Biodiversitätsuntersuchungen. - Bültmann, H., Fartmann, T., Hasse, T. [Hrsg.]: Trockenrasen auf unterschiedlichen Betrachtungsebenen - Berichte einer Tagung vom 26.-28. August in Münster. - Arb. Inst. Landschaftsökol. Münster 15: 73-81, Münster.

Dengler, J., Allers, M.-A. (2006): Plant species richness of the central European landscape on different spatial scales measured with a new approach. - Verh. Ges. Ökol. 36: 159, Berlin.

Dengler, J., Boch, S. (2006): Vegetation des NSG Kalkberg in Lüneburg. - Härdtle, W., Horst, K., Prüter, J. [Hrsg.]: Flora und Vegetation im nordöstlichen Niedersachsen. - Jahrb. Naturwiss. Ver. Fürstentum Lüneburg Sonderh. 1: 49-55, Lüneburg.

Dengler, J., Löbel, S., Boch, S. (2006): Dry grassland communities of shallow, skeletal soils (SedoScleranthenea) in northern Europe. - Tuexenia 26: 159-190, 6 Tab., Göttingen.

Diekmann, M., Kühne, A., Isermann, M. (2007): Random vs Non-Random Sampling: Effects on Patterns of Species Abundance, Species Richness and Vegetation-Environment Relationships. - Folia Geobot. 42: 179-191, Průhonice.

DierschKe, H (1994): Pflanzensoziologie. - 683 S., Eugen Ulmer, Stuttgart.

Dierssen, K. (1990): Einführung in die Pflanzengesellschaft. - 241 S., Wissenschaftliche Buchgesellschaft, Darmstadt.

Dobson, F. S. (2000): Lichens: An illustrated guide to the British and Irish species. - 431 S., Richmond Publ., Slough.

DolNIK, C. (2003a): Artenzahl-Areal-Beziehungen von Wald- und Offenlandgesellschaften - Ein Beitrag zur Erfassung der botanischen Artenvielfalt unter besonderer Berücksichtigung der Flechten und Moose am Beispiel des Nationalparks Kurischen Nehrung (Russland). - Mitt. Arbeitsgem. Geobot. SchleswigHolstein Hamb. 62: 183 S., Kiel.

DolniK, C. (2003b): Erfassung der Artenvielfalt auf Standardflächen am Beispiel der Phytodiversität. - Kiel. Not. Pflanzenkd. Schleswig-Holstein Hamb. 31: 72-83, Kiel.

DolnIK, C. (2006): Artenreichtum in Küsten-Sandtrockenrasen der Kurischen Nehrung und des Samlandes auf unterschiedlichen räumlichen Skalenebenen. - Bültmann, H., Fartmann, T., Hasse, T. [Hrsg.]: Trockenrasen auf unterschiedlichen Betrachtungsebenen - Berichte einer Tagung vom 26.-28. August in Münster. - Arb. Inst. Landschaftsökol. Münster 15: 83-95, Münster.

Drachenfels, O. VON (2004): Kartierschlüssel für Biotoptypen in Niedersachsen unter besonderer Berücksichtigung der nach $\S 28 \mathrm{a}$ und $\S 28 \mathrm{~b}$ NnatG geschützten Biotope sowie der Lebensraumtypen von Anhang I der FFH-Richtlinie, 6. Fassung. - Naturschutz Landschaftspflege Niedersachsen A/4, NLÖ, Hildesheim.

Drakare, S., Lennon, J. J., Hillebrand, H. (2006): The imprint of the geographical, evolutionary and ecological context on species-area relationships. - Ecol. Lett. 9: 215-227, Oxford.

Driesner, T. (1988): Geologie des Kalkbergs, in Der Kalkberg in Lüneburg, S. 5-7, BUND, Lüneburg.

Dunger, W., Fiedler, H. J. [Hrsg.] (1997): Methoden der Bodenbiologie. - 539 S., Gustav Fischer Verl., Jena.

Dytham, C. (2003): Choosing and Using Statistics - A Biologist's Guide. - 2. Aufl., XIII + 248 S., Blackwell, Malden [u. a.]

Eisele, W., ZäHringer, E. (1997): Vorläufiger Schlüssel zur Bestimmung der Seggen (Carex spec.) BadenWürttembergs nach vegetativen Merkmalen. - Flor. Rundbr. Beih. 5: 26 S., Verl. E. Golze, Göttingen.

Ellenberg, H. (1996): Vegetation Mitteleuropas mit den Alpen in ökologischer, dynamischer und historischer Sicht. - 5. Aufl., 1096 S., Ulmer, Stuttgart.

Ellenberg, H., Weber, H. E., Düll, R., Wirth, V., Werner, W., Paulissen, D. (1991): Zeigerwerte von Pflanzen in Mitteleuropa. - Scr. Geobot. 18: 248 S., Goltze, Göttingen.

Fitschen, J. (1994): Gehölzflora. - 10. Aufl., Quelle \& Meyer Verl., Wiesbaden.

Frahm, J.-P., Frey, W. (1992): Moosflora. - UTB 1250: 3. Aufl., 528 S., Ulmer, Stuttgart.

Fridley, J. D., Peet, R. K., Wentworth, T. R., White, P. S. (2005): Connecting fine- and broad-scale speciesarea relationships of southeastern U. S. flora. - Ecology 86: 1172-1177, Washington, DC.

Garve, E. (1994): Atlas der gefährdeten Farn- und Blütenpflanzen in Niedersachsen und Bremen. Kartierung 
1982-1992. - Naturschutz Landschaftspflege Niedersachsen 30: 2 Bd., 897 S., Niedersächsisches Landesamt für Ökologie, Hannover.

Garve, E. (2004): Rote Liste und Florenliste der Farn- und Blütenpflanzen in Niedersachsen und Bremen -

5. Fassung, Stand 1. 3. 2004. - Informationsdienst Naturschutz Niedersachsen 24: 1-76, Hildesheim.

Glavac, V. (1996): Vegetationsökologie - Grundfragen, Aufgaben, Methoden. - 358 S., Gustav Fischer, Jena.

Gleason, H. A. (1922): On the relation between species and area. - Ecology 3: 158-162.

GleAson, H. A. (1925): Species and area. - Ecology 6: 66-74.

GosLeE, S. C. (2006): Behaviour of vegetation sampling methods in the presence of spatial autocorrelation. J. Plant Ecol. 187: 203-212, Nanxincun [u. a.]

Grime, J. P. (1998): Benefits of plant diversity to ecosystems: immediate filter and founder effects. - J. Ecol. 86: 902-910, Oxford.

Haeupler, H., Schönfelder, P. (1989) [Hrsg.]: Atlas der Farn- und Blütenpflanzen der Bundesrepublik Deutschland. - 2. Aufl., 768 S., Ulmer, Stuttgart.

Haeupler, H., Muer, T. (2000): Bildatlas der Farn- und Blütenpflanzen Deutschlands. - Haeupler, H. [Hrsg.]: Die Farn- und Blütenpflanzen Deutschlands 2: 757 S., Ulmer, Stuttgart.

HANF, M. (1990): Ackerunkräuter Europas mit ihren Keimlingen und Samen. - 3. Aufl., 496 S., BLV Verlagsgesellschaft mbH, München.

Haversath, J.-B. (1997): Deutschland - Der Norden. - 193 S., Westermann Schulbuchverlag, Braunschweig.

He, F., Legendre, P. (1996): On species-area relations. - The American Naturalist 148: 719-737.

Henningsen, D., Katzung, G. (2002): Einführung in die Geologie Deutschlands. - 6. Aufl., VIII + $214+$ XVI S., Spektrum Akademischer Verl., Heidelberg [u. a.].

Новонм, С. (1998): Pflanzensoziologie und die Erforschung der Artenvielfalt - Überarbeitete und erweiterte Fassung der an der Universität Lüneburg eingereichten und angenommenen Habilitationsschrift. - Arch. Naturwiss. Diss. 5: 231 S., Galunder, Wiehl.

Новонм, C. (2000): Biodiversität. 214 S., Quelle \& Meyer Verl., Wiebelsheim.

Horst, K. (1983): Der „Kalkberg“ in Lüneburg - Refugium wärmeliebender und anderer seltener Pflanzen. - Eine floristisch-vegetationskundliche Dokumentation. - Jahrb. Naturwiss. Ver. Fürstentum Lüneburg 36: 197-234, Lüneburg.

JäGER, E. J., Werner, K. (2005) [Hrsg.]: Gefäßpflanzen: Kritischer Band. - Rothmaler, W. [Begr.]: Exkursionsflora von Deutschland 4: 10. Aufl., 980 S., Spektrum Akademischer Verl., Heidelberg [u. a.].

Koperski, M., Sauer, M., Braun, W. \& Gradstein, R. (2000): Referenzliste der Moose Deutschlands. 519 S., Schriftenreihe für Vegetationskunde, Heft 34, Bundesamt für Naturschutz 2000, Bonn - Bad Godesberg.

KowariK, I. (2003): Biologische Invasionen - Neophyten und Neozoen in Mitteleuropa. - 380 S., Ulmer, Stuttgart.

KüHn, I., BrandL, R., KLotz, S. (2004): The flora of German cities is naturally species rich. - Evol. Ecol. Res. 6: 749-764.

Lancken, H. von der (1997): Lage, naturräumliche Einheiten und Klima. - CoRdes, H., Kaiser, T., LancKen, H. von der, LütKepohl, M., Prüter, J. [Hrsg.]: Naturschutzgebiet Lüneburger Heide - Geschichte Ökologie - Naturschutz: 11-18, Verl. H. M. Hauschild, Bremen.

Landesamt für Bergbau, Energie und Geologie (2007): Startseite des LEBG Kartenservers. - URL: http://www.lbeg.de/grundwasser/produkte/kartenserver_startseite.htm [15.02.2007].

LaNDESVERMESSUNG UND GEOBASISINFORMATION NiEDERSAChSEN (2003): TOP50 Niedersachsen /Bremen - Version 4.0. - CD-ROM, Landesvermessung und Geobasisinformation Niedersachsen, Hannover.

LANDwEHR, J. (1966): Atlas van de nederlandse Bladmossen. - 504 S., ERLA, Amsterdam - Zuid.

LePš, J. (2005): Diversity and ecosystem function. - MAAREL, E. VAN DER [Hrsg.]: Vegetation Ecology: 199237, Blackwell Publishing, Oxford [u. a.].

LÉvêQue, C., Mounolou, J.-C. (2003): Biodiversity. 296 S., Wiley, Chichester.

Lomolino, M. V. (2000): Ecology's most general, yet protean pattern: the species-area relationship. - J. Biogeogr. 27: 17-26, Oxford.

LozÁn, J. L., Kausch, H. (2004): Angewandte Statistik für Naturwissenschaftler. - 3. Aufl., 300 S., Wissenschaftliche Auswertungen, Hamburg.

Lütkepohl, M., Hanstein, U. (1997): Spuren der Geschichte. - Cordes, H., Kaiser, T., Lancken, H. von der, 
LÜTKEPOHL, M., PrÜTER, J. [Hrsg.]: Naturschutzgebiet Lüneburger Heide - Geschichte - Ökologie Naturschutz: 11-18, Verl. H. M. Hauschild, Bremen.

MaARel, E. van Der (2005): Vegetation ecology - an overview. - MaArel, E. van DeR [Hrsg.]: Vegetation Ecology: 1-51, Blackwell Publishing, Oxford [u. a.].

Malcolm, B., Malcolm, N. (2000): Mosses and other bryophytes - an illustrated glossary. - 220 S., MicroOptics Pr., Nelson.

Meynen, E., Schmithüsen, J., Gellert, J., Neef, E., Müller-Miny, H., Schultze, J. H. (1962): Handbuch der naturräumlichen Gliederung Deutschlands. -2 Bd., 1339 S., 1 Kt., Bundesanstalt für Landeskunde und Raumforschung, Bonn.

Michaelis, L ., Menten, M. (1913): Die Kinetik der Invertinwirkung. - Biochemiche Zeitschrift 49: 333-369.

Muller, F. M. (1978): Seedlings of the North-Western European Lowland. - 654 S., Dr. W. Junk B.V. Publ., The Hague, Boston.

Mueller-Dombois, D., Ellenberg, H. (1974): Aims and methods of vegetation ecology. - XI + 547 S. + Beilage, Wiley, New York [u. a.].

NiEdERSÄChSISCHES LANDESAMT FÜR STATISTIK (2007): Bevölkerungsveränderungen in den kreisfreien Städten und Landkreisen im November $2006 . \quad$ URL: http://www.nls.niedersachsen.de/Tabellen/Bevoelkerung/Bev veraend 11 06.html [09.03.2007].

Oberdorfer, E. (2001): Pflanzensoziologische Exkursionsflora für Deutschland und angrenzende Gebiete. 8. Aufl., 1051 S., Ulmer, Stuttgart.

Palmer, M. W., White, P. S. (1994): On the existence of ecological communities. - J. Veg. Sci. 5: 279-282, Uppsala.

Peet, R. K., Wentworth, T. R., White, P. S. (1998): A flexible, multipurpose method for recording vegetation composition and structure. - Castanea 63: 262-274.

Pharo, E. J., Beattie, A. J., Binns, D. (1999): Vascular Plant Diversity as a Surrogate for Bryophyte and Lichen Diversity. - Conserv. Biol. 13: 282-292, Boston, Mass.

Pimm, S. L., Russell, G. J., Gittelman, J. L., Brooks, T. M. (1995): The future of biodiversity. - Science 269: 347-350, Washington, D. C.

Ротт, R. (1999): Lüneburger Heide - Wendland und Nationalpark Mittleres Elbtal. - 256 S., Verl. Eugen Ulmer, Stuttgart.

Preising, E., Weber, H. E., Vahle, H.-C. (2003): Die Pflanzengesellschaften Niedersachsens Bestandsentwicklung, Gefährdung und Schutzprobleme - Wälder und Gebüsche. - Naturschutz Landschaftspflege Niedersachsen 20(2). - 139 S., Niedersächsisches Landesamt für Ökologie, Hildesheim.

Primack, R. B. (1995): Naturschutzbiologie. - 713 S., Spektrum Akademischer Verl., Heidelberg.

Pullin, A. S. (2002): Conservation Biology. - 345 S., University Press, Cambridge.

Purvis, O. W., Coppins, B. J., Hawkswort, D. L., James, P. W., Moore, D. M. (1992) [Hrsg.]: The Lichen Flora of Great Britain and Ireland. - IX +710 S., British Lichen Society, London.

Reinhardt, U. (1982): Ur- und Frühgeschichte. - Kühlhorn, E. [Hrsg.]: Historisch-Landeskundliche Exkursionskarte von Niedersachsen - Blatt Lüneburg - Erläuterungsheft: 6-18, Kommissionsverlag August Lax, Hildesheim.

Rosenzweig, M. L. (1995): Species diversity in space and time. - XXI + 436 S., Cambridge Univ. Pr., Cambridge [u. a.].

Scheiner, S. M. (2003): Six types of species-area curves. - Global Ecol. Biogeogr. 12: 441-447, Oxford.

Scheiner, S. M. (2004): A mélange of curves - further dialogue about species-area relationships. - Global Ecol. Biogeogr. 13: 479-484, Oxford.

Schläpfer, F., Schmid, B., Seidl, I. (1999): Expert estimates about affects of biodiversity on ecosystem processes and services. - Oikos 52: 3-18.

Sснмітт, J. A. (1999): Neues zum Informationsgehalt von Arten/Areal-Kurven - Die Ermittlung von ArtenDiversität R, Minimum-Areal M und Mittlerer Arten-Densität D aus Teilflächen-Untersuchungen eines Gebietes über die Statistische, Hyperbolische, Kumulative Arten/Areal-Kurve am Beispiel Höherer Pilze. - Abh. Delattinia 25: 67-210, Saarbrücken.

Schulz, F., Dengler, J. (2006) [Hrsg.]: Verbreitungsatlas der Moose in Schleswig-Holstein und Hamburg. 402 S., Landesamt für Natur und Umwelt des Landes Schleswig-Holstein, Flintbek. 
Semmel, A. (1984): Geomorpholgie der Bundesrepublik Deutschland. - 4. Aufl., 192 S., Franz Steiner Verl. Wiesbaden, Stuttgart.

Sмiтн, A. J. (2006): The moss flora of Britain and Ireland. - 2. Aufl., 1012 S., Cambridge University Press, Cambridge.

Stohlgren, T. J., Falkner, M. B., Schell, L. D. (1995): A Modified-Whittaker nested vegetation sampling method. - Vegetatio 117: 113-121, Dordrecht [u. a.].

Thompson, K., Hodgson, J. G., Smith, R. M., Warren, P. H., Gaston, K. J. (2004): Urban domestic gardens (III): Composition and diversity of lawn floras. - J. Veg. Sci. 15: 373-378, Uppsala.

TuøRve, E. (2003): Shapes and functions of species-area curves: a review of possible models. - J. Biogeogr. 30: 827-835, Oxford.

Tonne, F. (1954): Besser Bauen mit Besonnungs- und Tageslichtplanung.- 41 S., Verlag Karl Hoffmann Schorndorf.

Turner, W. R., Tృørve, E. (2005): Scale-dependence in species-area relationships. - Ecography 28: 721-730, Copenhagen.

Tutin, T.G. (1980): Umbellifers of the British Isles. - 197 S., B.S.B.I. Handbook, No. 2, Botanical Society of the British Isles, London.

VDLUFA (1991): Die Untersuchung von Böden. - VDLUFA-Methodenbuch, Band I, VDLUFA-Verl., Darmstadt.

Vitousek, P. M., D'Antonio, C. M., Loope, L. L., Rejmánek, M., Westbrooks, R. (1997): Introduced species: A significant component of human-caused global change. - New Zealand Journal of Ecology 21: 1-16.

VölKsEN, G. (1984): Die Lüneburger Heide - Entstehung und Wandel einer Kulturlandschaft. - 52 S., Kommissionsverlag Göttinger Tageblatt, Göttingen \& Hannover.

WaniA, A., KÜHN, I., KLOTZ, S. (2005): Plant richness pattern in agricultural and urban landscapes in Central Germany - spatial gradients of species richness. - Landscape Urban Plann. 75"97-110.

Wellinghausen, N. (1997): Farnpflanzen. - 7. Aufl., 109 S., Deutscher Jugendbund für Naturbeobachtung, Hamburg.

Winter, R. Beckel, L. (1992) [Hrsg.]: Geo Satellitenbild Atlas Deutschland. - 2. Aufl., 239 S., Reise- und Verkehrsverlag, Berlin [u. a.].

WILDI, O . (1986): Analyse vegetationskundlicher Daten - Theorie und Einsatz statistischer Methoden. - 232 S., Veröff. Geobot. Inst. Eidg. Tech. Hochsch. Stift. Rübel 90: 226 S., Zürich.

Williamson, M. (2003): Species-area relationships at small scales in continuum vegetation. - J. Ecol. 2003: 904-907, Oxford.

Williamson, M., Gaston, K. J., Lonsdale, W. M. (2001): The species-area relationship does not have an asymptote! - J. Biogeogr. 28: 827-830, Oxford.

WIRTH, V. (1995): Flechtenflora. - UTB 1062: 2. Aufl., 661 S., Ulmer, Stuttgart.

Wisskirchen, R., Haeupler, H. (1998): Standardliste der Farn- und Blütenpflanzen Deutschlands. - Haeupler, H. [Hrsg.]: Die Farn- und Blütenpflanzen Deutschlands 1: 765 S., Ulmer, Stuttgart. 


\section{Tabellenverzeichnis}

Tabelle 1: Verwendete Kürzel für die unterschiedenen Flächengrößen und die Anzahl der Replikate

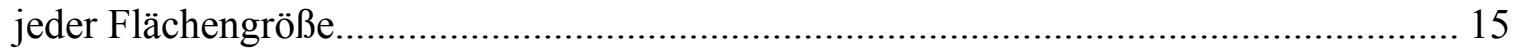

Tabelle 2: Definitionen verwendeter Größen deskriptiver Statisitik sowie des Bestimmtheitsmaßes.

Tabelle 3: Name, Funktion und Quelle der im Rahmen der Untersuchung zur Arten-ArealBeziehung getesteten Kurven.

Tabelle 4: Arithmetisches Mittel (Ø), Standardabweichung (SD), Median (Med.), Minimum (Min.) und Maximum (Max.) der Artenzahlen insgesamt, differenziert nach Artengruppen, Statusgruppen sowie der gepflanzten Arten.

Tabelle 5: Vergleich mittlerer Artenzahlen von Gefäßpflanzen auf unterschiedlichen Flächengrößen.

Tabelle 6: Vergleich mittlerer Gesamtartenzahlen auf unterschiedlichen Flächengrößen......

Tabelle 7: Schiefe (obere Tab.) und Kurtosis (untere Tab.) der Gesamtarten-, Gefäßpflanzenarten-, Flechtenarten- und Moosartenzahlen sowie der floristischen Gruppen (mit indigen Arten $=$ Ind., Neophyten $=$ Neo., Archaephythen $=$ Arch. und kultivierten Pflanzen $=$ Kult.) auf den unterschiedlichen Flächengrößen.

Tabelle 8: Arithmetisches Mittel, Standardabweichung, Minimum und Maximum zweier Trockenrasenverbände (Boch 2005) sowie die Bereiche, die $99 \%$ der Werte bei einer unterstellten Normalverteilung enthalten würden.

Tabelle 9: Mittleres Bestimmtheitsmaß und Signifikanzniveau der Exponentialfunktion der Standardabweichung der Artendichten insgesamt auf den verschiedenen Landschaftstypen sowie der Artengruppen.....

Tabelle 10: Mittleres Bestimmtheitsmaß und Signifikanzniveau der Exponentialfunktion und der polynomischen Funktion des mittleren Variationskoeffizienten der verschiedenen Landschaftstypen sowie der Artengruppen mit den Werten der Funktionsparameter der Exponentialfunktion.

Tabelle 11: Standardabweichung (SD) und Variationskoeffizient (V) von 50 Stichproben je Flächengröße, die notwendige Anzahl von Replikaten bei einer bestimmten Genauigkeit bei der Erfassung der Artendichte sowie die erzielte Genauigkeit mit 50 Stichproben; SE $=$ relativer Standardfehler.

Tabelle 12: Bestimmtheitsmaß (R2), relativer Extrapolationsfehler (rel. EE), mittlerer relativer Residuenfehler ( $\varnothing$ rel. RE) und maximaler relativer Residuenfehler (max. rel. RE) der getesteten Funktionen über alle Plots $(\mathrm{n}=50)$ gemittelt mit Standardabweichung.......... 58

Tabelle 13: Ranking des Bestimmtheitsmaßes mit Mittelwert (Ø) und Standardabweichung (SD) getrennt nach dem Raum, in dem die Kurven approximiert wurden.

Tabelle 14: Frequenz der Rangpositionen der getesten Funktionen im logarithmierten und nichtlogarithmierten Raum für die Gütekriterien mittlerer relativer Fehler ( $\varnothing$ rel. E), maximaler relativer Fehler (max. rel. E) und relativer Extrapolationsfehler (rel. EE), sowie deren Mittelwert (Ø) und Standardabweichung (SD). Die maximal erreichten Frequenzen jedes Kriteriums und jeden Ranges sind fett gedruckt.

Tabelle 15: Mittleres Ranking der Funktionen für alle Kriterien mit Standardabweichung, sowie der Mittelwert über alle Kriterien

Tabelle 16: Mittelwert, Schiefe, Kurtosis und p-Wert eines Kolmogorov-Smirnoff-Testes (p KS) sowie homogene Gruppen der z-Werte $(\mathrm{n}=50)$.

Tabelle 17: Mittlere z-Werte der Artenzahl-Areal-Kurven differenziert nach Landschaftstypen sowie das arithmetische Mittel über alle Flächenübergänge. Die angegeben homogenen Gruppen beziehen sich auf die z-Werte der Artenzahlen auf den Waldflächen........ 
Tabelle 18: Mittlere z-Werte auf den berücksichtigten Flächenübergängen differenziert nach

Artengruppen und floristischen Statusgruppen. Angegeben sind arithmetisches Mittel und sofern vorhanden Standardabweichung sowie das arithmetische Mittel über alle

Flächenübergänge $(\mathrm{n}=50)$.

Tabelle 19: Z-Werte der Gefäßpflanzenartenzahlen in verschiedenen Untersuchungsgebieten bei zufallsveteilten Stichproben, sowie das arithmetische Mittel über die vier Flächen von 0,01 m2 bis $100 \mathrm{~m} 2$. (1) Diekmann et al. (2007), (2) Chiarucci et al. (2006), (3) Crawley

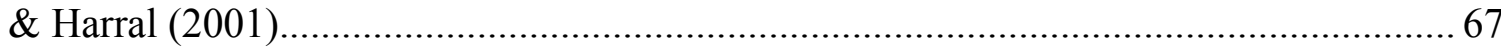

Tabelle 20: Mittlere z-Werte der Gesamtartenzahlen in verschiedenen Untersuchungsgebieten bei zufallsveteilten Stichproben

Tabelle 21: Prozentualer Anteil der Frequenz der häufigsten Arten bei zunehmender Flächengröße, sortiert nach den Prozenten der Artenfrequenzen auf der Fläche A7.....

Tabelle 22: Frequenzen der 20 häufigsten Arten der vorliegenden Studie im Vergleich mit den Angaben von Dengler \& Allers (2006) in Brandenburg mit AG = Artengruppe, $\mathrm{FS}=$ floristische Statusgruppe und $\mathrm{F}=$ Frequenz [\%].

Tabelle 23: Die häufigsten Gefäßpflanzenarten der vorliegenden Untersuchung und in Brandenburg Dengler \& Allers (2006) entsprechend, mit deren Rang (R), Messtischblattfrequenz (M),

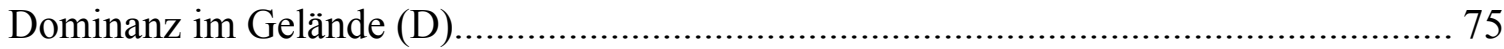

\section{Abbildungsverzeichnis}

Abbildung 1: Lage des Untersuchungsgebietes in einer Karte aus Winter \& Beckel (1992).............. 4

Abbildung 2: Lufttemperatur in Lüneburg aus Müller \& Westermeier (1996)................................5

Abbildung 3: Luftfeuchte in Lüneburg aus Müller \& Westermeier (1996).................................... 5

Abbildung 4: Niederschlag in Lüneburg aus Müller \& Westermeier (1996)..................................6

Abbildung 5: Sonnenschein und Bewölkung in Lüneburg aus Müller \& Westermeier (1996)........... 6

Abbildung 6: Das Untersuchungsgebiet im Nordosten Niedersachsens aus

TOP50 Niedersachsen/Bremen (Landesvermessung und Geobasisinformation

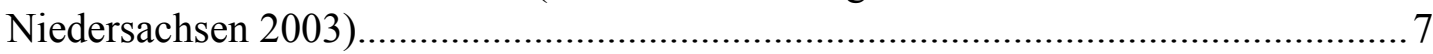

Abbildung 7: Schematische Darstellung des Aufnahmedesigns (nicht maßstabsgetreu)..................16

Abbildung 8: Diagrammscheibe für $53^{\circ}$ nördlicher Breite......................................................... 20

Abbildung 9: Box-Whisker-Plot der absoluten Artenzahlen auf allen Flächengrößen. Ausgenommen der Fläche A7 $(n=50)$ gilt: $n=200$. Die Ausreißer sind größer bzw. kleiner als die Ober- bzw. Unterwerte der Box $+1 *$ Box-Bereich. Die Extremwerte berechnen sich durch die Summe der Ober- bzw. Unterwerte der Box + 2*Box-Bereich...................31

Abbildung 10: Mittlere Artendichten und Standardabweichung der Flechten, Moose und Gefäßpflanzen differenziert nach Landschaftstypen de Fläche A7.

Abbildung 11: Mittlere Artendichten und Standardabweichung der indigenen Arten, Neophyten, Archaeophyten und kultivierten Arten differenziert nach Landschaftstypen der Fläche A7. Berücksichtigt wurden nur Gefäßpflanzen.

Abbildung 12: Artendichte der Neopyhten in Abhängigkeit zur Gesamtartendichte der Fläche A7.41

Abbildung 13: Histogramm der absoluten Artenzahlen der Fläche A6 mit Normalverteilungskurve.

Abbildung 14: Histogramm der logarithmierten Artenzahlen der Fläche A6 mit Normalverteilungskurve.

Abbildung 15: Histogramm der arcus-sinus-transformierten Artenzahlen der Fläche A6 mit Normalverteilungskurve.

Abbildung 16: Histogramm der absoluten Artenzahlen der Flechten der Fläche A6 mit

Normalverteilungskurve...

: Mittlerer Artenanteil der Flechten, Moose und Gefäßpflanzen in Abhängigkeit zur 
Flächengröße.

Abbildung 18: Mittlere Verteilung der Moose $\left(\mathrm{M}^{*}\right)$ und Flechten $\left(\mathrm{F}^{*}\right)$ auf die Substrat-Straten

Boden $\left({ }^{*} \mathrm{~B}\right)$, Epiphyten $\left({ }^{*} \mathrm{E}\right)$, Totholz $\left({ }^{*} \mathrm{~T}\right)$ und Stein $\left({ }^{*} \mathrm{~S}\right)$ bei zunehmender

Flächengröße.

Abbildung 19: Mittlere Artenanteile der Artengruppen differenziert nach den verschiedenen

Landschaftstypen in Abhängigkeit zur Flächengröße.

Abbildung 20: Mittlerer Anteil der floristischen Statusgruppen (s. Kap. 3.3 für Definitionen) in

Abhängigkeit zur Flächengröße.

Abbildung 21: Mittlere Artenanteile der floristischen Statusgruppen differenziert nach den verschiedenen Landschaftstypen in Abhängigkeit zur Flächengröße.

Abbildung 22: Mittlerer Artenanteil wildwachsender und gepflanzter Neophyten in Abhängigkeit zur Flächengröße im Siedlungsbereich.

Abbildung 23: Mittlere Standardabweichung der Artenzahl auf unterschiedlich großen Flächen bei logarithmierter Flächengröße unter Berücksichtigung aller Flächengrößen (A) und unter Ausschluss der Fläche A7 (B).

Abbildung 24: Mittlerer Variationskoeffizient der Artenzahl in Abhängigkeit zur logarithmierten Flächengröße

Abbildung 25: Doppelt logarithmische Darstellung der Artenzahl-Arealgröße-Wertepaare aller Plots.

Abbildung 26: Kurvenrverlauf der getesteten Funktionen exemplarisch dargestellt anhand eines Plots.

Abbildung 27: Mittlere z-Werte der Gesamtartenzahlen auf allen Plots mit Standardabweichung...65

Abbildung 28: Häufigkeitsverteilung der Arten im Untersuchungsgebiet bei logarithmierter

Skalierung der Ordinatenachse.

Abbildung 29: Muster der Häufigkeitsverteilung der Arten auf den 50 Plots.

\section{Abkürzungsverzeichnis}

$\begin{array}{llll}\text { Abb. } & \text { Abbildung } & \mathrm{n} & \text { Anzahl } \\ \text { Aufl. } & \text { Auflage } & \mathrm{NN} & \text { Normal Null } \\ \text { GPS } & \text { Global Positioning System } & \mathrm{R}^{2} & \text { Erklärte Varianz } \\ \text { Hrsg. } & \text { Herausgeber } & \mathrm{SD} & \text { Standardabweichung } \\ \text { i. d. R. } & \text { in der Regel } & \text { Tab. } & \text { Tabelle } \\ \text { Jh. } & \text { Jahrhundert } & \text { Verl. } & \text { Verlag } \\ \text { Kap. } & \text { Kapitel } & \text { vgl. } & \text { vergleiche }\end{array}$




\section{Anhang 1: Verzeichnis der Pflanzensippen}

$\mathrm{FS}=$ Floristischer Status, Leb. $=$ Lebensform, RL D $=$ Rote Liste Deutschland, RL NI $=$ Rote Liste Niedersachsen, RL NIT $=$ Rote Liste niedersächsisches Tiefland, Frequenz $=$ Frequenz der Arten auf den $2009 \mathrm{~m}^{2}$ Flächen.

\section{Flechten}

\section{Wissenschaftlicher Artname}

Amandinea punctata

Aspicilia contorta

Bacidina arnoldiana

Caloplaca cf. Crenolatella

Caloplaca holocarpa

Caloplaca spec.

Candelariella aurella

Candelariella reflexa

Candelariella xanthostigma

Chaenotheca ferruginea

Cladonia cf. glauca

Cladonia cf. rei

Cladonia coniocraea

Cladonia digitata

Cladonia fimbriata

Cladonia furcata ssp. furcata

Cladonia glauca

Cladonia macilenta

Cladonia pyxidata

Dimerella pineti

Evernia prunastri

Graphis scripta

Hypocenomyce scalaris

Hypogymnia physodes

Krustenflechte indet

Krustenflechte indet

Krustenflechte indet 2

Lecanora albescens

Lecanora cf. Xanthostoma

Lecanora conizaeoides

Lecanora dispersa

Lecanora expallens

Lecanora hagenii

Lecanora muralis

Lecanora saligna

Lecanora spec.

Lecanora spec.

Lecidea spec.

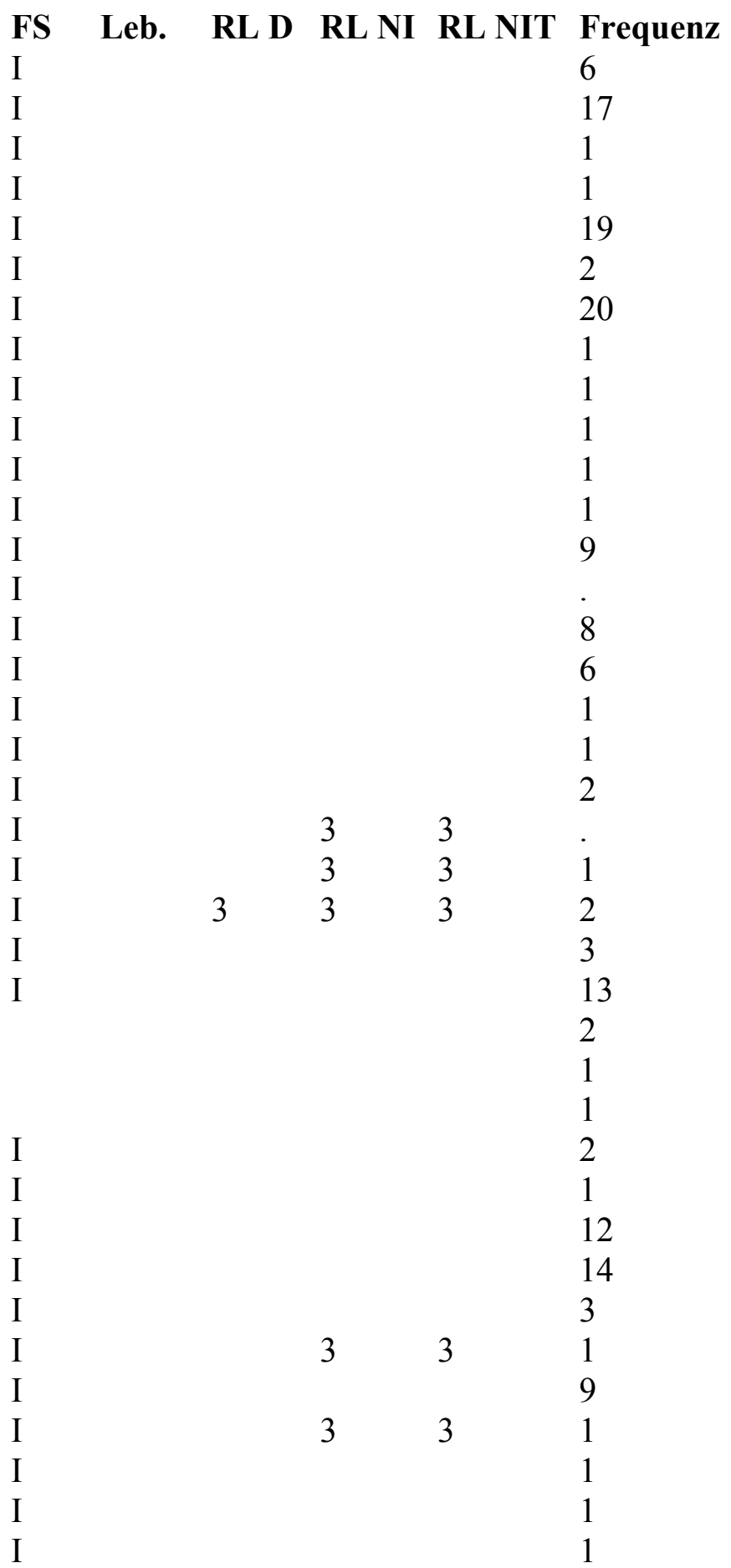


Wissenschaftlicher Artname

Lecidella elaeochroma

Lecidella stigmatea

Lepraria incana

Lepraria lobificans

Melanelia glabratula

Micarea denigrata

Micarea spec.

Parmelia saxatilis

Parmelia sulcata

Peltigera didactyla

Pertusaria leioplaca

Phaeophysica orbicularis

Phlyctis argena

Physcia adscendens

Physcia tenella

Placynthiella icmalea

Porina aenea

Trapeliopsis flexuosa

Trapeliopsis granulosa

Verrucaria nigrescens

Xanthoria candelaria

Xanthoria fulva

Xanthoria parietina

Xanthoria polycarpa
FS

I
RL D RL NI RL NIT Frequenz

3

2

2

12

13

2

2

2

1

7

1

$\begin{array}{llll}3 & 1 & 1 & 1\end{array}$

$3 \quad 3 \quad 1$

3

14

2

4

2

1

1

10

6

\section{Gefäßpflanzen}

\section{Wissenschaftlicher Artname}

Abies alba

Acer campestre

Acer negundo

Acer palmatum

Acer platanoides

Acer pseudoplatanus

Achillea millefolium agg.

Aegopodium podagraria

Aesculus hippocastanum

Agave spec.

Agave spec. 2

Agrostis capillaris

Agrostis gigantea

Agrostis stolonifera

Agrostis vinealis

Aira caryophyllea ssp. caryophyllea

Alcea rosea

Alchemilla mollis

Alchemilla spec.

Alliaria petiolata
FS Leb. RL D RL NI RL NIT Frequenz

$\begin{array}{llll}\text { I } & \mathrm{P} & 3 & 1\end{array}$

$\begin{array}{lll}\mathrm{I} & \mathrm{P} & 10\end{array}$

$\mathrm{N} \quad \mathrm{P}$

$\mathrm{N}-1$

$\begin{array}{lll}\mathrm{I} & \mathrm{P} & 7\end{array}$

$\begin{array}{lll}\mathrm{I} & \mathrm{P} & 19\end{array}$

I 14

$\begin{array}{lll}\text { I } & \mathrm{G}, \mathrm{H} & 6\end{array}$

$\mathrm{N}-3$

$\mathrm{N}-1$

$\mathrm{N}-1$

$\begin{array}{lll}\mathrm{I} & \mathrm{H} & 51\end{array}$

$\begin{array}{lll}\mathrm{I} & \mathrm{H} & 8\end{array}$

$\begin{array}{lll}\text { I } & \mathrm{H} & 10\end{array}$

$\begin{array}{lll}\text { I } & \mathrm{H} & 8\end{array}$

$\begin{array}{lllll}\mathrm{I} & \mathrm{T} & \mathrm{V} & \mathrm{V} & 3\end{array}$

$\mathrm{N}-1$

$\mathrm{N}-1$

$\begin{array}{lll}\text { I } & \text { H }\end{array}$ 
Wissenschaftlicher Artname

Allium spec.

Alnus glutinosa

Alopecurus myosuroides

Alopecurus pratensis

Amelanchier asiatica

Amelanchier lamarckii

Ananas comosus

Anchusa arvensis

Anemone nemorosa

Anthemis cf. Tinctoria

Anthoxanthum aristatum

Anthriscus sylvestris

Apera spica-venti

Aquilegia vulgaris

Arabidopsis thaliana

Arabis caucasica

Arabis procurrens

Arabis $\mathrm{x}$ arendsii

Arenaria serpyllifolia

Arrhenatherum elatius

Artemisia campestris

Artemisia cf. Abrotanum

Artemisia vulgaris

Aruncus dioicus

Aster spec.

Astilbe chinensis

Astilbe spec.

Athyrium filix-femina

Begonia spec.

Bellis perennis

Bellis spec.

Berberis spec.

Berberis thunbergii

Berteroa incana

Betula pendula

Boswellia spec.

Brachypodium sylvaticum ssp. sylvaticum

Brassica napus

Brassica nigra

Brassica oleracea

Briza media

Bromus hordeaceus

Bromus sterilis

Bromus tectorum

Brunnera macrophylla

Buxus sempervirens

Calamagrostis epigejos

Calendula officinalis

Calla palustris
FS Leb. RL D RL NI RL NIT Frequenz

$\mathrm{N} \quad 1$

$\begin{array}{lll}\mathrm{I} & \mathrm{P} & 2\end{array}$

A $\mathrm{T} \quad 1$

I $\mathrm{H} \quad 2$

$\mathrm{N}-1$

$\mathrm{N} \quad \mathrm{N} \quad 1$

$\mathrm{N}$

A $\quad \mathrm{T}, \mathrm{H}$

I $\quad \mathrm{G}$

$\mathrm{N}-1$

$\begin{array}{lll}\mathrm{N} & \mathrm{T} & 3\end{array}$

I $\mathrm{H} \quad 6$

I T 3

I $\mathrm{H} \quad 1$

A $\mathrm{T}, \mathrm{H}$

$\mathrm{N} 2$

$\mathrm{N}-4$

1

I T,C 12

$\begin{array}{lll}\mathrm{I} & \mathrm{H} & 12\end{array}$

$\begin{array}{lllll}\mathrm{I} & \mathrm{C} & \mathrm{V} & \mathrm{V}\end{array}$

$\mathrm{N}-1$

$\begin{array}{lll}\mathrm{I} & \mathrm{H}, \mathrm{C} & 18\end{array}$

I $\mathrm{H} \quad 1$

\# 1

$\mathrm{N}-1$

$\mathrm{N}$

I $\mathrm{H} \quad 1$

$\mathrm{N}-3$

$\begin{array}{lll}\text { A } & \mathrm{H} & 7\end{array}$

I

$\mathrm{N}-1$

$\mathrm{N} \quad \mathrm{T}, \mathrm{H}$

I P

$\mathrm{N}-1$

$\mathrm{H} \quad 4$

U 13

$\mathrm{N} \quad \mathrm{T} \quad 4$

I $\quad \mathrm{C} \quad 4$

$\begin{array}{llll}\mathrm{I} & \mathrm{H} & \mathrm{V} & 2\end{array}$.

I $\mathrm{T} \quad 2$

A $\mathrm{T} \quad 7$

A $\mathrm{T} \quad 2$

$\mathrm{U} / \mathrm{N}$

I $\quad \mathrm{N} 2$

$\begin{array}{lll}\text { I } & \mathrm{G}, \mathrm{H} & 7\end{array}$

$\begin{array}{lllll}\mathrm{K} & \mathrm{A}, \mathrm{G} & 3 & 3 & 3\end{array}$ 
Wissenschaftlicher Artname

Calluna vulgaris

Caltha palustris

Calystegia sepium ssp. sepium

Campanula glomerata ssp. Glomerata

Campanula persicifolia

Campanula poscharskyana

Cannabis sativa

Capsella bursa-pastoris

Cardamine hirsuta

Cardamine pratensis agg.

Cardamine spec.

Carduus nutans ssp. nutans

Carex acuta

Carex acutiformis

Carex arenaria

Carex cf. arenaria

Carex cf. Distans

Carex cf. pilulifera

Carex cf. remota

Carex hirta

Carex muricata agg.

Carex pilulifera

Carex remota

Carex spec.

Carex sylvatica

Carpinus betulus

Centaurea dealbata

Centaurea montana

Cerastium arvense

Cerastium glomeratum

Cerastium holosteoides

Cerastium semidecandrum

Cerastium spec.

Ceratocapnos claviculata

Ceratostigma plumbaginoides ${ }^{\wedge} \mathrm{KG}$

Cf. Agrostis

Cf. Aster spec.

Cf. Calamagrostis arundinacea

Cf. Cardamine pratensis

Cf. Caryopteris spec.^SG

Cf. Hypericum

Cf. Lamium

Cf. Senecio spec.

Cf. Stellaria

Chamaecyparis nootkatensis ${ }^{\wedge} \mathrm{SG}$

Chamaecyparis obtusa ${ }^{\wedge} \mathrm{SG}$

Chamaecyparis pisifera ${ }^{\wedge} \mathrm{SG}$

Chamaecyparis spec.^SG

Chelidonium majus
FS Leb. RL D RL NI RL NIT Frequenz

I Z

I $\mathrm{H}$

I $\quad \mathrm{G}, \mathrm{H}$

I $\mathrm{H}$

I H

$\mathrm{N}$

$\mathrm{N}$

I T

I $\quad \mathrm{T}, \mathrm{H}$

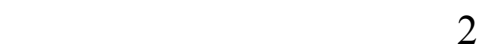

$\begin{array}{llll}3 & 3 & 4\end{array}$

(

2

$2 \quad 0 \quad 1$

$\mathrm{A} / \mathrm{I} \quad \mathrm{H}$

I G,A

G,A

$\mathrm{G}, \mathrm{H}$

I

I

I

I

I

I $\quad \mathrm{H}$

I $\mathrm{H}$

I

I

I $\quad \mathrm{H}$

I $\quad \mathrm{P}$

$\mathrm{N}$

I

I C

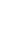

$\mathrm{A} / \mathrm{I} \quad \mathrm{T}$

2

I $\quad$ C,H

10

I T,H 15

I 2

I Tli 3

$\mathrm{N}$

1

1

2

$\mathrm{R}$

\section{F}

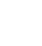

$\mathrm{N}$

N

$\mathrm{N}$

N

I $\mathrm{H}$ 
Wissenschaftlicher Artname

Chelone obliqua ${ }^{\wedge} \mathrm{KG}$

Chenopodium album

Chenopodium strictum

Circaea lutetiana

Cirsium arvense

Cirsium palustre

Cirsium vulgare

Clematis alpina ${ }^{\wedge} \mathrm{KG}$

Clematis vitalba

Convallaria majalis

Convolvulus arvensis

Conyza canadensis

Coreopsis grandiflora ${ }^{\wedge} \mathrm{KG}$

Cornus $\operatorname{mas}^{\wedge} \mathrm{SG}$

Cornus sericea ${ }^{\wedge} \mathrm{SG}$

Corydalis cava

Corylus avellana

Corynephorus canescens

Cotoneaster franchetii^ ${ }^{\wedge} \mathrm{SG}$

Cotoneaster horizontalis ${ }^{\wedge} \mathrm{KG}$

Cotoneaster rotundifolius ${ }^{\wedge} \mathrm{KG}$

Cotoneaster spec.^KG

Cotoneaster spec.^S

Crataegus monogyna var. monogyna

Crataegus rhipidophylla

Crepis spec.

Cupressus macrocarpa ${ }^{\wedge} \mathrm{SG}$

Cupressus sempervirens ${ }^{\wedge} \mathrm{SG}$

Dactylis glomerata ssp. glomerata

Dahlia spec. ${ }^{\wedge} \mathrm{KG}$

Danthonia decumbens

Datura arborea ${ }^{\wedge} \mathrm{SG}$

Daucus carota ssp. carota

Delphinium spec.^KG

Deschampsia cespitosa

Deschampsia flexuosa

Deutzia longifolia ${ }^{\wedge} \mathrm{SG}$

Dicentra spectabilis` $\mathrm{KG}$

Digitaria ischaemum

Dryopteris carthusiana

Dryopteris dilatata

Dryopteris filix-mas agg.

Echinochloa crus-galli

Elymus repens

Epilobium lamyi

Epilobium tetragonum

Epipactis helleborine

Equisetum arvense

Equisetum fluviatile
FS Leb. RL D RL NI RL NIT Frequenz

$\mathrm{N}$

$\mathrm{A} / \mathrm{I} \quad \mathrm{T} \quad 24$

N $\mathrm{T} \quad 6$

$\begin{array}{lll}\mathrm{I} & \mathrm{G} & 8\end{array}$

I $\mathrm{G} \quad 8$

I $\mathrm{H} \quad 1$

$\begin{array}{lll}\mathrm{I} & \mathrm{H} & 7\end{array}$

I Nli 1

I Pli 1

I G 2

I $\mathrm{G}, \mathrm{H} \quad 5$

$\begin{array}{lll}\mathrm{N} & \mathrm{T}, \mathrm{H} & 18\end{array}$

$\mathrm{N}-1$

$\begin{array}{llll}\mathrm{I} & \mathrm{N}, \mathrm{P} & 3 & 1\end{array}$

$\mathrm{N}-1$

I G 1

I N 3

I H 6

$\mathrm{N}-1$

$\mathrm{U} / \mathrm{N}$

$\mathrm{N}-3$

$\mathrm{N}-1$

$\mathrm{N}-2$

I N,P 4

I 6

I 2

$\mathrm{N}$

$\mathrm{N}$

I H 24

$\mathrm{N}-1$

$\begin{array}{lllll}\text { I } & \text { H } & \text { V } & \text { V } & 3\end{array}$

$\mathrm{N}$

I $\quad \mathrm{H} \quad 4$

$\mathrm{N} 2$

$\begin{array}{lll}\mathrm{I} & \mathrm{H} & 8\end{array}$

$\begin{array}{lll}\text { I } & \mathrm{H} & 10\end{array}$

$\mathrm{N}-1$

$\begin{array}{lll}\text { I } & \mathrm{H} & 23\end{array}$

I H 1

I 3

A $\mathrm{T} \quad 2$

$\begin{array}{lll}\text { I } & \mathrm{T} & 50\end{array}$

I 1

I 1

I G 2

I $\mathrm{G} \quad 2$

I $\quad \mathrm{A}, \mathrm{G} \quad 1$ 
Wissenschaftlicher Artname

Equisetum palustre

Equisetum spec.

Erica cinerea ${ }^{\wedge} \mathrm{KG}$

Erodium cicutarium

Erophila verna

Erysimum cheiri ${ }^{\wedge} \mathrm{KG}$

Erysimum hybridum ${ }^{\wedge} \mathrm{KG}$

Euonymus europaea

Euonymus fortunei ${ }^{\wedge} \mathrm{SG}$

Euonymus hamiltonianus ${ }^{\wedge} \mathrm{S}$

Euonymus phellomanus ${ }^{\wedge} \mathrm{SG}$

Eupatorium cannabinum

Fagus sylvatica

Fallopia convolvulus

Fallopia japonica

Festuca altissima

Festuca arundinacea

Festuca brevipila

Festuca gigantea

Festuca nigrescens

Festuca ovina agg.

Festuca rubra agg.

Festuca spec.

Filipendula ulmaria

Fragaria vesca

Frangula alnus

Frangula alnus ${ }^{\wedge} \mathrm{S}$

Fraxinus excelsior

Galeobdolon argentatum

Galeobdolon luteum

Galeopsis bifida

Galeopsis cf. tetrahit

Galeopsis spec.

Galeopsis speciosa

Galeopsis tetrahit

Galinsoga ciliata

Galinsoga parviflora

Galium album

Galium aparine

Galium palustre ssp. palustre

Gartenpflanze indet ${ }^{\wedge} \mathrm{KG}$

Geranium macrorrhizum ${ }^{\wedge} \mathrm{KG}$

Geranium molle

Geranium psilostemon^ ${ }^{\wedge} \mathrm{KG}$

Geranium pusillum

Geranium robertianum ssp. robertianum

Geranium spec. ${ }^{\wedge} \mathrm{KG}$

Gerbera spec.^KG

Geum chiloense ${ }^{\wedge} \mathrm{KG}$
FS Leb. RL D RL NI RL NIT Frequenz

I G 6

$\begin{array}{llll}2 & 2 & 5\end{array}$

$\begin{array}{llll}\text { I } & \text { Z } & 1 & 1\end{array}$

I $\mathrm{T}, \mathrm{H}$

I 2

A $\mathrm{C}-1$

\#

I N 3

$\mathrm{N}-1$

$\mathrm{N}-1$

1

I $\mathrm{H} \quad 1$

$\begin{array}{lll}\text { I } & \mathrm{P} & 29\end{array}$

A Tli 9

N $\mathrm{G} \quad 2$

I H 3

I

I $\mathrm{H} \quad 2$

I H 2

I H

I 12

I

I

I $\quad \mathrm{H}$

I $\mathrm{H}$

I N

I $\mathrm{N}$

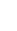

12

2

11

12

1

4

$\begin{array}{lll}\mathrm{V} & \mathrm{V} & 1\end{array}$

I T

I T

3

2

$\mathrm{I} \quad \mathrm{T}-5$

I

I Tli 23

I H 3

\#

$\mathrm{N} 2$

$\begin{array}{lll}\mathrm{I} & \mathrm{T} & 4\end{array}$

$\mathrm{N}-1$

A $\mathrm{T} \quad 6$

I $\mathrm{T}, \mathrm{H} \quad 5$ 
Wissenschaftlicher Artname

Geum urbanum

Glechoma hederacea

Glyceria maxima

Gymnocarpium dryopteris

Hedera helix

Helianthemum spec.^KG

Helianthus annuus

Helianthus spec.^KG

Heliopsis spec. ${ }^{\wedge} \mathrm{KG}$

Hemerocallis lilioasphodelus ${ }^{\wedge} \mathrm{KG}$

Heracleum sphondylium ssp. sphondylium I

Herniaria glabra ssp. glabra

Heuchera spec. ${ }^{\wedge} \mathrm{KG}$

Hieracium cf. caespitosum

Hieracium lachenalii

Hieracium laevigatum

Hieracium pilosella

Hieracium spec.

Holcus lanatus

Holcus mollis

Hordeum vulgare

Hosta fortune $\mathrm{i}^{\wedge} \mathrm{KG}$

Hosta plantaginea ${ }^{\wedge} \mathrm{KG}$

Humulus lupulus ${ }^{\wedge} \mathrm{S}$

Hydrangea macrophylla^ $\mathrm{KG}$

Hydrangea sargentiana ${ }^{\wedge} \mathrm{SG}$

Hypericum calycinum ${ }^{\wedge} \mathrm{KG}$

Hypericum perforatum

Hypochaeris radicata

Ilex aquifolium

Impatiens noli-tangere

Impatiens parviflora

Impatiens walleriana ${ }^{\wedge} \mathrm{KG}$

Iris pseudacorus

Iris spec.^KG

Juglans regia ${ }^{\wedge} \mathrm{SG}$

Juncus articulatus

Juncus effusus

Juncus tenuis

Keimling indet.

Keimling indet. 2

Kolkwitzia amabilis`SG

Laburnum $x$ watereri^ ${ }^{\wedge} \mathrm{SG}$

Lactuca serriola

Lamium album ssp. album

Lamium amplexicaule

Lapsana communis

Larix kaempferi

Lathyrus odoratus ${ }^{\wedge} \mathrm{KG}$
FS Leb. RL D RL NI RL NIT Frequenz

I $\mathrm{H} \quad 7$

$\begin{array}{lll}\text { I } & \mathrm{G}, \mathrm{H} & 14\end{array}$

I $\mathrm{A}, \mathrm{H} \quad 4$

$\begin{array}{llll}\text { I } & \mathrm{G} & 3 & 1\end{array}$

I Z,P 13

$\mathrm{K}$

\# 1

$\mathrm{N}-1$

$\mathrm{N}-3$

$\mathrm{N}$

I 2

I $\mathrm{H} \quad 3$

I H 2

I H 1

I 2

I $\mathrm{H} \quad 43$

$\begin{array}{lll}\text { I } & \mathrm{G}, \mathrm{H} & 22\end{array}$

K 17

$\mathrm{N} 23$

$\mathrm{N}-1$

I Hli 1

$\mathrm{N} 2$

$\mathrm{N}-1$

$\mathrm{N}$

I $\mathrm{H} \quad 17$

$\begin{array}{lll}\mathrm{I} & \mathrm{H} & 12\end{array}$

$\begin{array}{lll}\mathrm{I} & \mathrm{P} & 7\end{array}$

I T 1

N T 11

$\mathrm{N}-1$

I $\quad \mathrm{A}, \mathrm{G} \quad 5$

\# 1

$\mathrm{N} \quad \mathrm{P}-1$

I $\mathrm{H} \quad 1$

$\begin{array}{lll} & \mathrm{H} & 8\end{array}$

$\mathrm{N} \quad \mathrm{H} \quad 1$

$\mathrm{N}-1$

$\mathrm{N}-1$

I H,T 1

A $\mathrm{H} \quad 2$

A $\mathrm{T} \quad 1$

I H,T 3

$\mathrm{N} 25$

4

(n)

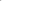

4

(1)

4

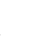

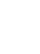

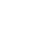

(1)

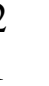

5 
Wissenschaftlicher Artname

Lathyrus pratensis

Leontodon autumnalis

Leontodon hispidus

Ligustrum vulgare ${ }^{\wedge} \mathrm{SG}$

Lilium martagon ${ }^{\wedge} \mathrm{KG}$

Lilium spec. ${ }^{\wedge} \mathrm{KG}$

Lobelia erinus ${ }^{\wedge} \mathrm{KG}$

Lolium perenne

Lonicera periclymenum

Lonicera $\mathrm{x}$ brownii ${ }^{\wedge} \mathrm{KG}$

Lupinus polyphyllus ${ }^{\wedge} \mathrm{KG}$

Luzula pilosa

Lychnis viscaria ${ }^{\wedge} \mathrm{KG}$

Lycopus europaeus

Lysimachia nummularia

Lysimachia vulgaris

Lythrum salicaria

Maianthemum bifolium

Malus domestica ${ }^{\wedge} \mathrm{SG}$

Malva alcea ${ }^{\wedge} \mathrm{KG}$

Malva moschata ${ }^{\wedge} \mathrm{KG}$

Matricaria discoidea

Matricaria recutita

Medicago lupulina

Melica uniflora

Melissa officinalis ${ }^{\wedge} \mathrm{KG}$

Mentha x piperita

Menyanthes trifoliata $^{\wedge} \mathrm{KG}$

Mimulus guttatus ${ }^{\wedge} \mathrm{KG}$

Mimulus moschatus

Molinia arundinace ${ }^{\wedge} \mathrm{KG}$

Molinia caerulea

Mycelis muralis

Myosotis arvensis

Myosotis spec.^KG

Nuphar pumila^KG

Oenothera parviflora

Oenothera spec.

Omphalodes verna

Oplopanax spec.^SG

Ornithopus perpusillus

Oxalis acetosella

Pachysandra terminalis ${ }^{\wedge} \mathrm{KG}$

Paeonia officinalis ${ }^{\wedge} \mathrm{KG}$

Papaver spec. ${ }^{\wedge} \mathrm{KG}$

Paris quadrifolia

Pelargonium peltatum ${ }^{\wedge} \mathrm{KG}$

Pennisetum alopecuroides ${ }^{\wedge} \mathrm{KG}$

Persicaria hydropiper

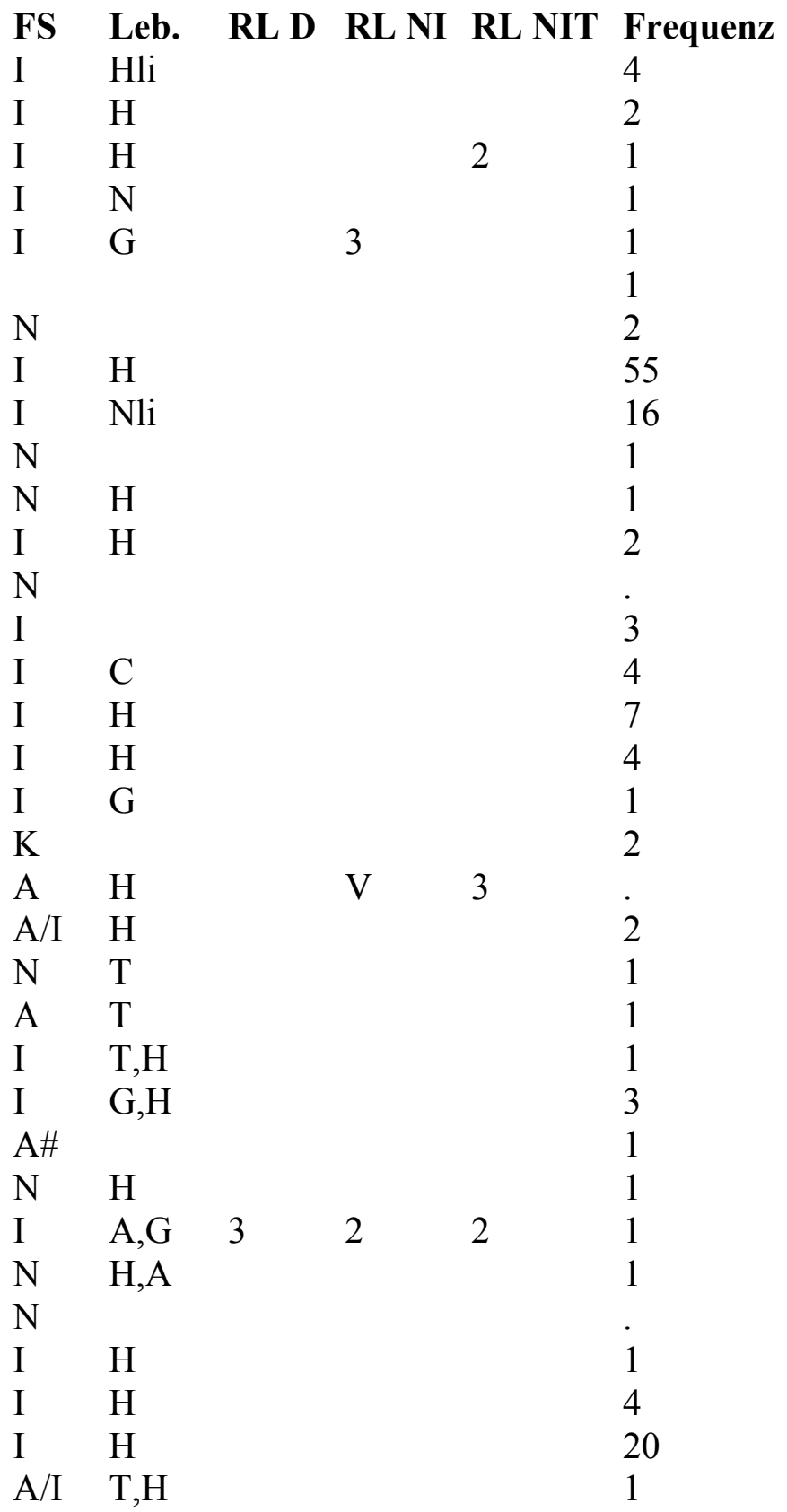

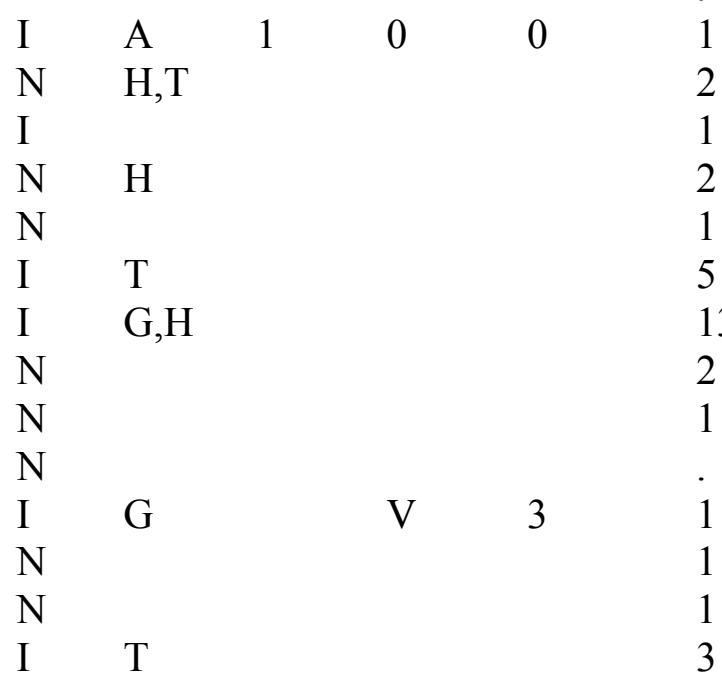


Wissenschaftlicher Artname

Persicaria lapathifolia ssp. pallida

Phacelia tanacetifolia ${ }^{\wedge} \mathrm{KG}$

Phalaris arundinacea

Phleum bertolonii

Phlox paniculata ${ }^{\wedge} \mathrm{KG}$

Phragmites australis

Picea abies

Picea omorica ${ }^{\wedge} \mathrm{SG}$

Picea sitchensis ${ }^{\wedge} \mathrm{BG}$

Pieris spec. ${ }^{\wedge} \mathrm{SG}$

Pinus sylvestris

Plantago lanceolata

Plantago major ssp. intermedia

Plantago major ssp. major

Poa angustifolia

Poa annua

Poa compressa

Poa nemoralis

Poa palustris

Poa pratensis

Poa spec.

Poa trivialis ssp. trivialis

Poaceae indet.

Polemonium caeruleum ${ }^{\wedge} \mathrm{KG}$

Polygonatum multiflorum

Polygonum arenastrum

Polygonum aviculare

Portulaca oleracea ssp. oleracea

Potentilla anserina

Potentilla argentea agg.

Potentilla erecta

Potentilla fruticosa ${ }^{\wedge} \mathrm{SG}$

Primula elatior ${ }^{\wedge} \mathrm{KG}$

Primula vulgaris

Prunella vulgaris

Prunus avium

Prunus cf. spinosa

Prunus domestica ${ }^{\wedge} \mathrm{SG}$

Prunus serotina

Prunus spec.

Prunus tenella^${ }^{\wedge} \mathrm{SG}$

Pseudolysimachion spicatum ${ }^{\wedge} \mathrm{KG}$

Pseudotsuga menziesii ${ }^{\wedge} \mathrm{B}$

Pteridium aquilinum

Pulmonaria officinalis ${ }^{\wedge} \mathrm{KG}$

Quercus petraea

Quercus robur

Ranunculus acris

Ranunculus repens

\begin{tabular}{|c|c|c|c|c|c|}
\hline FS & Leb. & RL D & RL NI & RL NIT & Frequenz \\
\hline I & $\mathrm{T}$ & & & & 4 \\
\hline $\mathrm{K}$ & & & & & 5 \\
\hline I & $\mathrm{G}, \mathrm{H}$ & & & & 6 \\
\hline I & $\mathrm{H}$ & & & & 1 \\
\hline $\mathrm{N}$ & & & & & 4 \\
\hline I & $\mathrm{G}, \mathrm{A}$ & & & & 4 \\
\hline I & $\mathrm{P}$ & & & & 7 \\
\hline $\mathrm{N}$ & & & & & 1 \\
\hline $\mathrm{N}$ & & & & & 2 \\
\hline $\mathrm{N}$ & & & & & 1 \\
\hline I & $\mathrm{P}$ & & & & 38 \\
\hline A & $\mathrm{H}$ & & & & 17 \\
\hline I & $\mathrm{H}, \mathrm{T}$ & & & & 3 \\
\hline I & $\mathrm{H}$ & & & & 11 \\
\hline I & $\mathrm{H}, \mathrm{G}$ & & & & 24 \\
\hline I & $\mathrm{T}, \mathrm{H}$ & & & & 13 \\
\hline I & $\mathrm{H}$ & & & & 3 \\
\hline I & $\mathrm{H}$ & & & & 5 \\
\hline I & $\mathrm{H}$ & & & & 1 \\
\hline I & $\mathrm{H}, \mathrm{G}$ & & & & 14 \\
\hline I & & & & & 1 \\
\hline I & $\mathrm{H}, \mathrm{C}$ & & & & 16 \\
\hline I & & & & & . \\
\hline I & $\mathrm{H}$ & 3 & $\mathrm{R}$ & & . \\
\hline I & $\mathrm{G}$ & & & & 1 \\
\hline I & $\mathrm{T}$ & & & & 16 \\
\hline I & $\mathrm{T}$ & & & & 9 \\
\hline A & $\mathrm{T}$ & & & & 1 \\
\hline I & $\mathrm{H}$ & & & & 3 \\
\hline I & $\mathrm{H}$ & & & & 1 \\
\hline I & $\mathrm{H}$ & & & & 1 \\
\hline & & & & & 2 \\
\hline I & $\mathrm{H}$ & & & 3 & 3 \\
\hline I & $\mathrm{H}$ & 3 & 1 & 1 & 1 \\
\hline I & $\mathrm{H}$ & & & & 1 \\
\hline I & $\mathrm{P}$ & & & & 10 \\
\hline I & & & & & 1 \\
\hline $\mathrm{N}$ & & & & & 1 \\
\hline $\mathrm{N}$ & $\mathrm{N}, \mathrm{P}$ & & & & 16 \\
\hline & & & & & 15 \\
\hline $\mathrm{N}$ & & & & & 1 \\
\hline $\mathrm{N}$ & & & & & 2 \\
\hline $\mathrm{U} / \mathrm{N}$ & & & & & 2 \\
\hline $\mathrm{I}$ & $\mathrm{G}$ & & & & 1 \\
\hline I & $\mathrm{H}$ & & & & 1 \\
\hline I & $\mathrm{P}$ & & & & 8 \\
\hline I & $\mathrm{P}$ & & & & 48 \\
\hline I & $\mathrm{H}$ & & & & 3 \\
\hline I & $\mathrm{H}$ & & & & 1 \\
\hline
\end{tabular}


Wissenschaftlicher Artname

Ranunculus sceleratus

Raphanus raphanistrum

Rhododendron spec. ${ }^{\wedge} \mathrm{KG}$

Robinia pseudoacacia ${ }^{\wedge} \mathrm{B}$

Rosa canina

Rosa spec.

Rosa $\mathrm{x}$ centifolia ${ }^{\wedge} \mathrm{KG}$

Rubus caesius

Rubus corylifolius agg.

Rubus idaeus

Rubus spec.

Rudbeckia spec.^${ }^{\wedge} \mathrm{KG}$

Rumex acetosa

Rumex acetosella

Rumex crispus

Rumex obtusifolius

Rumex sanguineus

Rumex spec.

Rumex thyrsiflorus

Sagina procumbens

Sagittaria latifolia ${ }^{\wedge} \mathrm{KG}$

Salix aurita ${ }^{\wedge} \mathrm{KG}$

Salvia officinalis ${ }^{\wedge} \mathrm{KG}$

Sambucus nigra

Saxifraga spec.^KG

Scirpus sylvaticus

Scrophularia nodosa

Sedum acre

Sedum ochroleucum ${ }^{\wedge} \mathrm{KG}$

Sedum sexangulare ${ }^{\wedge} \mathrm{KG}$

Sedum spec.

Sedum spurium

Sedum telephium agg.

Senecio cf. erucifolius

Senecio jacobaea

Senecio sylvaticus

Setaria viridis

Silene latifolia ssp. alba

Sisymbrium officinale

Solanum rantonnetii ${ }^{\wedge} \mathrm{SG}$

Solanum spec.^KG

Solanum tuberosum

Solidago canadensis

Solidago gigantea

Sonchus asper ssp. asper

Sorbus aucuparia

Sorbus koehneana ${ }^{\wedge} \mathrm{SG}$

Spergularia rubra

Spiraea japonica`SG
FS Leb. RL D RL NI RL NIT Frequenz

I T

A $\mathrm{T}$

$3 \quad 3 \quad 1$

$\mathrm{N}$

I P

3

I N

1

$\mathrm{N}$

I zli

5

1

1

3

19

1

2

1

8

2

I $\mathrm{H}$

I $\quad \mathrm{C}, \mathrm{H}$

A

I N

A

I N

I

I G

3

I $\mathrm{H}$

5

3

N C

I C

6

1

9

1

$\begin{array}{lll}\mathrm{I} & \mathrm{H} & 7 \\ \mathrm{I} & \mathrm{T} & 2\end{array}$

$\mathrm{I} \quad \mathrm{T} \quad 2$

A $\mathrm{T}-3$

I $\mathrm{H} \quad 4$

A $\mathrm{T} \quad 2$

$\mathrm{N}-1$

$\mathrm{N} \quad \mathrm{H}, \mathrm{G} \quad 3$

N H,G

I $\mathrm{T} \quad 1$

$\begin{array}{lll}\mathrm{I}, \mathrm{N} & 19\end{array}$

$\mathrm{N}-1$

$\begin{array}{lll}\mathrm{A} / \mathrm{I} & \mathrm{T}, \mathrm{H} & 7\end{array}$

$\mathrm{N}-1+1$ 
Wissenschaftlicher Artname

Spiraea salicifolia

Spiraea spec.

Spiraea $\mathrm{x}$ arguta $^{\wedge} \mathrm{SG}$

Spiraea $x$ vanhouttei ${ }^{\wedge} S$

Stachys cf. byzantina ${ }^{\wedge} \mathrm{KG}$

Stachys sylvatica

Stellaria graminea

Stellaria holostea

Stellaria media

Syringa vulgaris

Tagetes spec. ${ }^{\wedge} \mathrm{KG}$

Tanacetum coccineum ${ }^{\wedge} \mathrm{KG}$

Tanacetum vulgare

Taraxacum sect. Ruderalia

Taraxacum spec.

Taxus baccata

Teesdalia nudicaulis

Thalictrum flavum

Thuja occidentalis ${ }^{\wedge} \mathrm{SG}$

Thuja orientalis ${ }^{\wedge} \mathrm{SG}$

Thuja spec. ${ }^{\wedge} \mathrm{KG}$

Tilia cordata

Torilis japonica

Trientalis europaea

Trifolium arvense ssp. arvense

Trifolium campestre

Trifolium dubium

Trifolium pratense

Trifolium repens

Trifolium spec.

Tripleurospermum perforatum

Triticum aestivum ${ }^{\wedge} \mathrm{KG}$

Urtica dioica

Vaccinium myrtillus

Verbascum nigrum

Verbascum spec.^ ${ }^{\wedge} \mathrm{G}$

Verbascum thapsus ssp. thapsus

Veronica arvensis

Veronica chamaedrys

Veronica montana

Veronica officinalis

Veronica polita

Veronica spec.

Vicia angustifolia

Vicia hirsuta

Vicia lathyroides

Vicia sativa agg.

Vicia spec.

Vicia tetrasperma

\section{FS Leb. RL D RL NI RL NIT Frequenz}

$\mathrm{N} \quad 1$

2

1

1

1

I H

I $\mathrm{H} \quad 1$

I $\mathrm{C} \quad 12$

$\begin{array}{lll}\mathrm{I} & \mathrm{T} & 46\end{array}$

$\mathrm{N} 2$

$\mathrm{N} \quad 6$

I $\mathrm{H} \quad 4$

I $\quad 37$

$\begin{array}{lllll}\mathrm{I} & \mathrm{P} & 3 & 3 & 3\end{array}$

I T,H 2

$\begin{array}{lllll}\mathrm{I} & \mathrm{H} & 3 & 3 & 4\end{array}$

$\mathrm{N} 2$

$\mathrm{N}-1+2$

$\mathrm{N}-1$

$\begin{array}{lll}\mathrm{I} & \mathrm{P} & 6\end{array}$

I T,H 2

I $\mathrm{G} \quad 9$

$\begin{array}{lll}\mathrm{I} & \mathrm{T} & 6\end{array}$

I $\mathrm{T}$

I T 3

I H 2

$\begin{array}{lll}\mathrm{C}, \mathrm{H} & 14\end{array}$

I 1

$\begin{array}{lll}\text { A } & \mathrm{T} & 2\end{array}$

K 6

$\begin{array}{lll}\mathrm{I} & \mathrm{H} & 18\end{array}$

$\begin{array}{lll}\text { I } & \mathrm{Z} & 8\end{array}$

I H 2

I 1

I $\quad \mathrm{H} \quad 1$

$\begin{array}{lll}\text { A } & \mathrm{T} & 20\end{array}$

I C

$\begin{array}{llll}\text { I } & \text { C } & \text { V } & 1\end{array}$

I $\quad \mathrm{C}$

$\begin{array}{llll}\mathrm{A} & \mathrm{T} & 3 & 1\end{array}$

3

13

I Tli $\quad 5$

$\begin{array}{lllll}\text { I } & \text { T,H } & 3 & 3 & 3\end{array}$

I

I 2

$\begin{array}{lll}\text { I } & \text { Tli } & 8\end{array}$ 
Wissenschaftlicher Artname

Viola arvensis

Viola biflora

Viola labradorica ${ }^{\wedge} \mathrm{KG}$

Viola odorata

Viola reichenbachiana

Viola spec.

Viola spec.^KG

Viola $\mathrm{x}$ wittrockiana ${ }^{\wedge} \mathrm{KG}$

Weigela spec. ${ }^{\wedge} \mathrm{KG}$

Yucca filamentosa ${ }^{\wedge} \mathrm{KG}$

Zea mays ${ }^{\wedge} \mathrm{KG}$
FS Leb. RL D RL NI RL NIT Frequenz

A/I T 13

I $\mathrm{H} \quad 2$

$\mathrm{N}-1$

$\begin{array}{lll}\mathrm{N} & \mathrm{H} & 2\end{array}$

I $\mathrm{H} \quad 1$

$\begin{array}{lll}\mathrm{V} & \mathrm{V} & 3 \\ & 2\end{array}$

K

$\mathrm{N}$

$\mathrm{N}$

K
2

1

2

4

\section{Moose}

Wissenschaftlicher Artname

Amblystegium serpens

Atrichum undulatum

Aulacomnium androgynum

Barbula convoluta

Barbula unguiculata

Brachythecium albicans

Brachythecium rutabulum

Brachythecium salebrosum

Bryum argenteum

Bryum barnesii

Bryum bicolor

Bryum capillare

Bryum klinggraeffii

Cephaloziella divaricata

Ceratodon purpureus ssp. purpureus

Dicranella heteromalla

Dicranella staphylina

Dicranoweisia cirrata $^{\wedge} \mathrm{E}$

Dicranum polysetum ${ }^{\wedge} \mathrm{X}$

Dicranum scoparium

Eurhynchium praelongum

Grimmia pulvinata

Hypnum cupressiforme var.

Cupressiforme

Hypnum cupressiforme var. lacunosum

Hypnum cupressiforme ${ }^{\wedge} \mathrm{E}$

Hypnum jutlandicum

Leucobryum glaucum

Lophocolea bidentata ${ }^{\wedge} \mathrm{X}$

Lophocolea heterophylla ${ }^{\wedge} \mathrm{E}$

Marchantia polymorpha

Mnium hornum

Orthotrichum affine ${ }^{\wedge} \mathrm{E}$
FS Leb. RL D RL NI RL NIT Frequenz

I

I $\quad \mathrm{C},(\mathrm{E})$

I

I

I

I

I

I

I C

C

3

4

12

5

12

$\mathrm{C},(\mathrm{E}) \quad 130$

$\mathrm{C} 2$

23

I 18

I 3

I $\mathrm{C} \quad 2$

I $\quad \mathrm{C},(\mathrm{E}) \quad 1$

I $\mathrm{C},(\mathrm{E}) \quad 22$

I $\mathrm{C} \quad 4$

I $\mathrm{C} \quad 2$

$\begin{array}{lll}\text { I } & \mathrm{C} & 12\end{array}$

I $\quad \mathrm{C} 2$

I $\mathrm{C},(\mathrm{E}) \quad 17$

I $\mathrm{C} \quad 12$

I 1

$\begin{array}{lll}\text { I } & \mathrm{C}, \mathrm{E} & 48\end{array}$

I C 3

I 1

$\begin{array}{lll}\mathrm{I} & \mathrm{C} & 8\end{array}$

I C 2

I $\mathrm{C} \quad 2$

$\begin{array}{lll}\mathrm{C}, \mathrm{H} & 10\end{array}$

I 5

I $\mathrm{H},(\mathrm{C}$

I $\mathrm{C}, \mathrm{E}$ 
Orthotrichum diaphanum ${ }^{\wedge} \mathrm{E}$

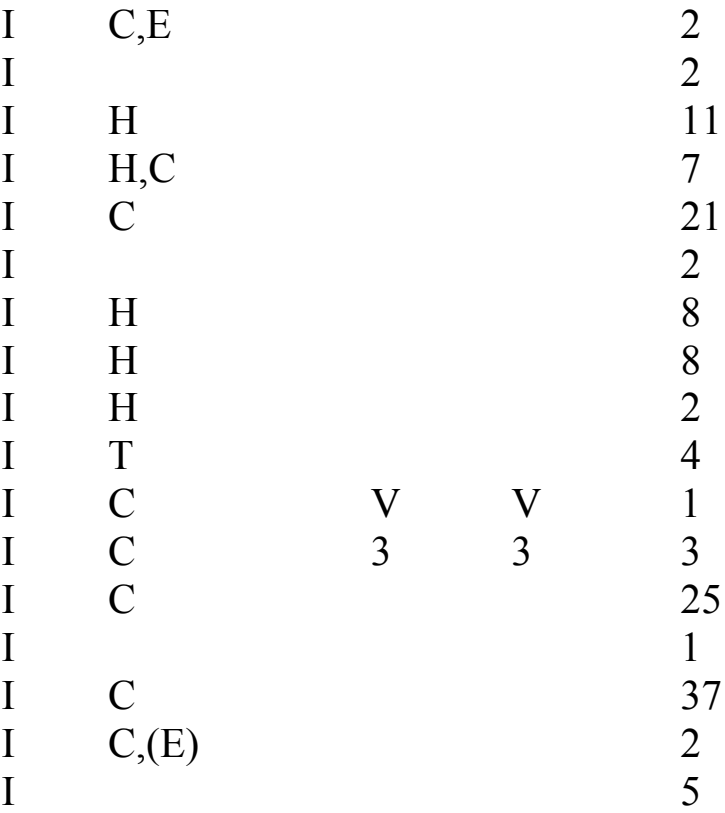

Phascum cuspidatum

Plagiomnium affine 1

Plagiomnium undulatum

Pleurozium schreberi

Pohlia cf. nutans

Polytrichum formosum

Polytrichum juniperinum

Polytrichum piliferum

Pottia truncata

Ptilidium ciliare

Racomitrium canescens ssp. canescens

Rhytidiadelphus squarrosus

Schistidium apocarpum agg. ${ }^{\wedge} \mathrm{L}$

Scleropodium purum

Tetraphis pellucida ${ }^{\wedge} \mathrm{X}$

Tortula muralis 


\section{Anhang 2: Plotkoordinaten}

$\begin{array}{lll}\text { Nr. } & \text { Rechtswert } & \text { Hochwert } \\ 01 & 3593328 & 5898313 \\ 02 & 3595522 & 5907238 \\ 03 & 3597991 & 5907977 \\ 04 & 3589162 & 5901701 \\ 05 & 3598688 & 5898825 \\ 06 & 3591828 & 5897665 \\ 07 & 3589421 & 5898934 \\ 08 & 3591552 & 5897339 \\ 09 & 3592196 & 5900997 \\ 10 & 3595176 & 5901197 \\ 11 & 3593672 & 5901145 \\ 13 & 1796887 & 2950293 \\ 14 & 3599760 & 5906310 \\ 15 & 3600083 & 5900193 \\ 16 & 3599679 & 5897902 \\ 17 & 3596741 & 5906340 \\ 18 & 3599801 & 5902508 \\ 19 & 3592289 & 5905520 \\ 21 & 3529867 & 4571975 \\ 22 & 3589733 & 5901089 \\ 23 & 3594399 & 5902899 \\ 24 & 3593028 & 5908166 \\ 25 & 3589466 & 5899670 \\ 26 & 3588962 & 5907401 \\ 27 & 3590175 & 5899983\end{array}$

$\begin{array}{lll}\text { Nr. } & \text { Rechtswert } & \text { Hochwert } \\ 29 & 3597793 & 5905280 \\ 30 & 3589996 & 5898591 \\ 31 & 3597385 & 5904212 \\ 32 & 3590928 & 5901646 \\ 33 & 3595114 & 5905147 \\ 34 & 3595230 & 5899245 \\ 35 & 3599740 & 5904751 \\ 36 & 3594796 & 5906074 \\ 37 & 3598019 & 5900194 \\ 38 & 3590905 & 5906789 \\ 39 & 3590369 & 5897798 \\ 40 & 3597397 & 5905473 \\ 41 & 3599867 & 5907633 \\ 42 & 3599070 & 5903378 \\ 43 & 3594528 & 5904712 \\ 44 & 3594529 & 5898837 \\ 45 & 3589360 & 5905986 \\ 46 & 3596408 & 5905406 \\ 47 & 3595569 & 5898923 \\ 48 & 3598945 & 5901508 \\ 49 & 3591296 & 5899445 \\ 50 & 3592746 & 5900824 \\ 51 & 3592258 & 5906079 \\ 55 & 3594795 & 5900059 \\ 62 & 3598749 & 5902212\end{array}$




\section{Anhang 3: Aufnahmebogen}

\section{Biodiversitäts-Untersuchung um Lüneburg (MTB 2728)}

Fläche $\mathrm{Nr}$ : Teilfläche: Datum: Lage:.

Gauß-Krüger: RW: $\mathrm{km} / \mathrm{HW}$ : km Exposition: Neigung: \% /

Nutzungsform: Sonnenscheindauer:

Landschaftsstruktur:

\begin{tabular}{|l|l|l|l|}
\hline & Deckung (\%) & $\varnothing$-Höhe $(\mathrm{m})$ & max. Höhe $(\mathrm{m})$ \\
\hline B & & & \\
\hline S & & & \\
\hline K & & & \\
\hline M & & & \\
\hline Vegetation Ges. & & & \\
\hline
\end{tabular}

\begin{tabular}{|l|l|l|l|}
\hline & Deckung (\%) & $\varnothing$-Tiefe $(\mathrm{m})$ & max. Tiefe $(\mathrm{m})$ \\
\hline $\mathrm{W}_{\mathrm{n}}$ [natant] & & & \\
\hline $\mathrm{W}_{\mathrm{s}}$ [submers] & & & \\
\hline Streu & & & \\
\hline Totholz & & - & - \\
\hline Gestein & & - & - \\
\hline
\end{tabular}

\begin{tabular}{|c|c|c|c|c|c|c|c|c|}
\hline \multirow[b]{2}{*}{ Nr. } & \multirow[b]{2}{*}{ Schicht } & \multirow[b]{2}{*}{ Art } & \multicolumn{6}{|c|}{ Kantenlänge } \\
\hline & & & $1 \mathrm{~cm}$ & $3 \mathrm{~cm}$ & $10 \mathrm{~cm}$ & $30 \mathrm{~cm}$ & $1 \mathrm{~m}$ & $3 \mathrm{~m}$ \\
\hline 1 & & & & & & & & \\
\hline 2 & & & & & & & & \\
\hline 3 & & & & & & & & \\
\hline 4 & & & & & & & & \\
\hline 5 & & & & & & & & \\
\hline 6 & & & & & & & & \\
\hline 7 & & & & & & & & \\
\hline 8 & & & & & & & & \\
\hline 9 & & & & & & & & \\
\hline 10 & & & & & & & & \\
\hline 11 & & & & & & & & \\
\hline 12 & & & & & & & & \\
\hline 13 & & & & & & & & \\
\hline 14 & & & & & & & & \\
\hline 15 & & & & & & & & \\
\hline 16 & & & & & & & & \\
\hline 17 & & & & & & & & \\
\hline 18 & & & & & & & & \\
\hline 19 & & & & & & & & \\
\hline 20 & & & & & & & & \\
\hline 21 & & & & & & & & \\
\hline 22 & & & & & & & & \\
\hline$\ldots$ & & & & & & & & \\
\hline
\end{tabular}


